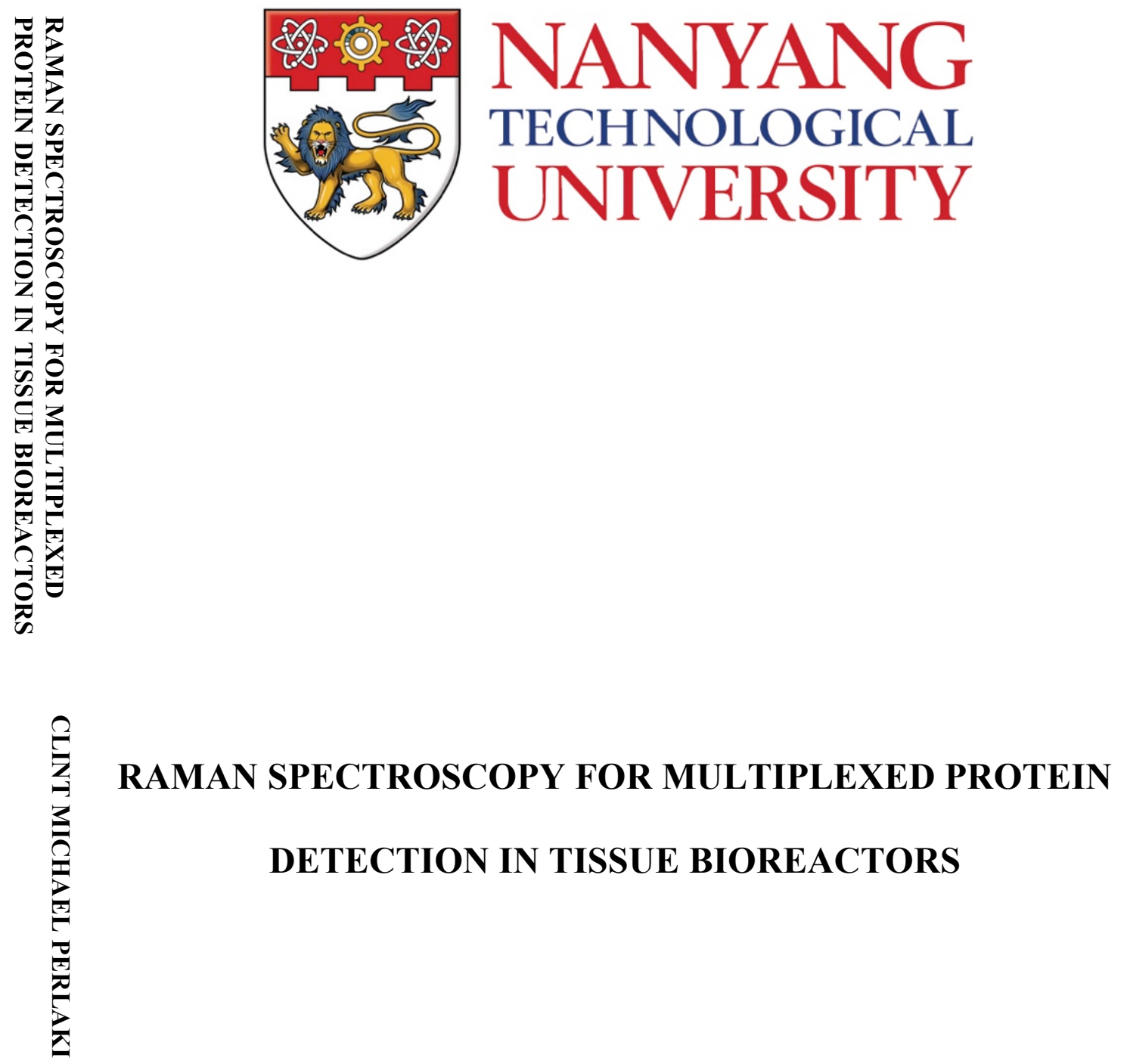

CLINT MICHAEL PERLAKI

SCHOOL OF CHEMICAL AND BIOMEDICAL ENGINEERING

$\stackrel{\cong}{\Xi}$ 


\section{RAMAN SPECTROSCOPY FOR MULTIPLEXED PROTEIN DETECTION IN TISSUE BIOREACTORS}

\section{Clint Michael Perlaki}

School of Chemical and Biomedical Engineering

A thesis submitted to the Nanyang Technological University in partial fulfillment of the requirement for the degree of Doctor of Philosophy 


\section{Acknowledgments}

I express my sincere gratitude to all who have helped me through this journey. I give special thanks to my supervisor, Dr. Liu Quan, for his steadfast dedication, empathy, flexibility, and inspiration despite the formidable challenges we encountered. Thanks also go to my co-supervisor, Dr. Sierin Lim, for her kind generosity in making her lab and expertise available. I also thank my first primary supervisor, Dr. Mayasari Lim, who warmly welcomed me into this program and kept me motivated by her enthusiasm during her last year in NTU. I am also immensely gratified by the time and effort volunteered to me by Dr. Alexander William Jackson at the Institute of Chemical and Engineering sciences. He not only contributed invaluable time and resources to our project, but also was a great mentor and friend. I thank Dr. He Tao of the same institute for his instrumental role in establishing our collaboration and his neighborly help. Lastly, I thank my family and friends for standing with me throughout this long adventure. 


\section{Table of Contents}

Chapter 1 Background .....................................................................................5

1.1 Raman Spectroscopy ....................................................................................5

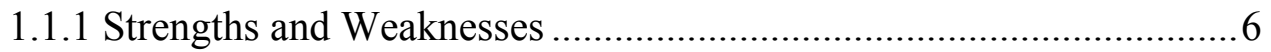

1.2 Polarized Raman Spectroscopy ...........................................................7

1.3 Raman Optical Activity .................................................................8

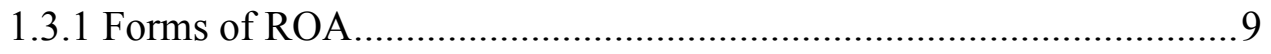

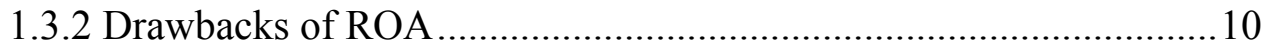

1.4 Surface Enhanced Raman Scattering (SERS)......................................12

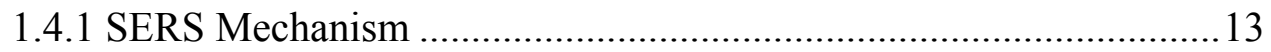

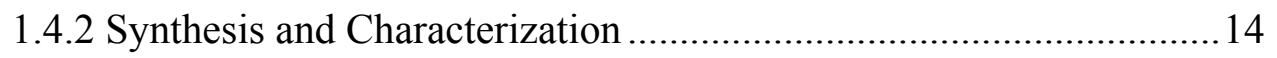

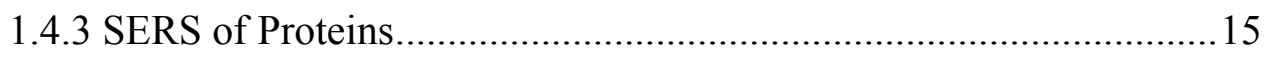

Chapter 2 Raman Spectroscopy Based Techniques in Tissue Engineering -

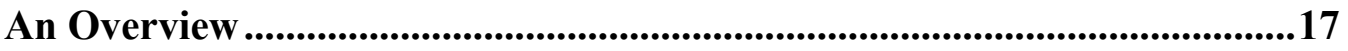

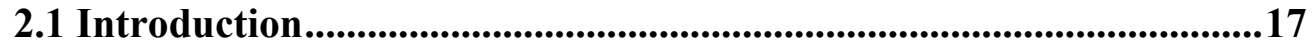

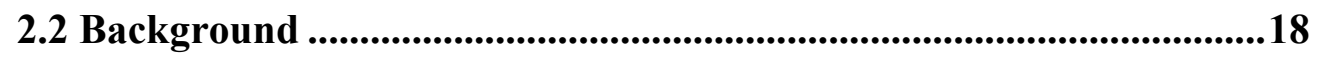

2.2.1 Monitoring process components in bioreactors ............................. 18

2.2.2 Role of RS for online monitoring in TERM .................................20

2.3 Applications of Raman spectroscopy to monitor target bioreactor components .........................................................................................................220

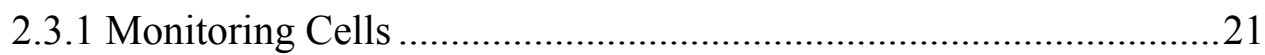

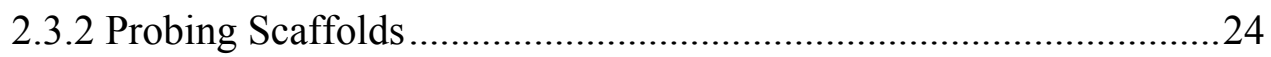

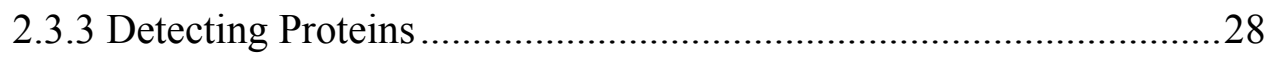

2.4 Conclusion and Future Prospects.................................................................46 
Chapter 3 Polarized Raman Spectroscopy for Enhanced Quantification of Protein Concentrations in an Aqueous Mixture ..........................................48

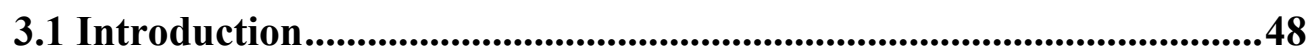

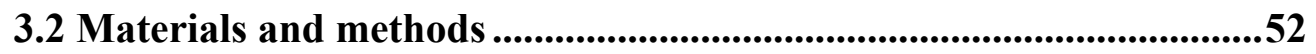

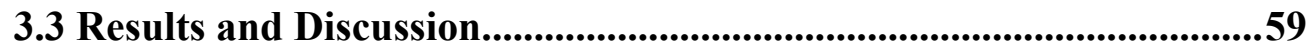

3.4 Conclusion

Chapter 4 Surface Enhanced Raman Optical Activity for Detection and Quantification of Aqueous Protein Mixtures ..............................................88

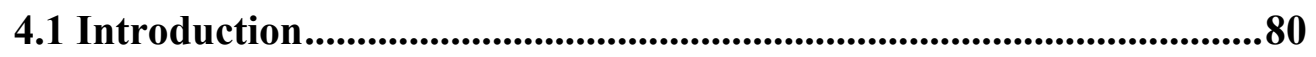

4.2 Construction and Calibration of ROA optical setup ............................81

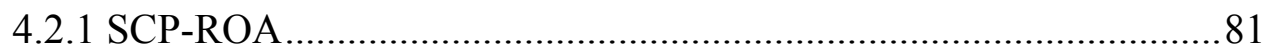

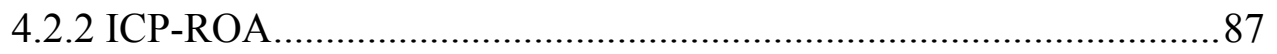

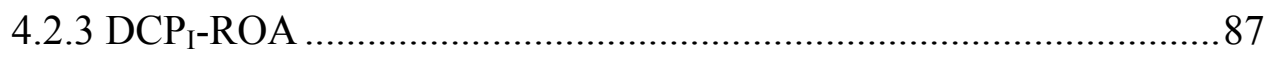

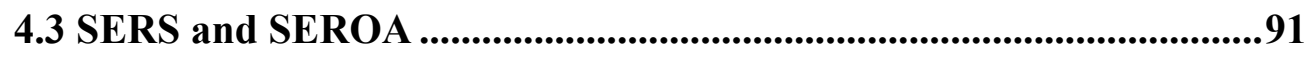

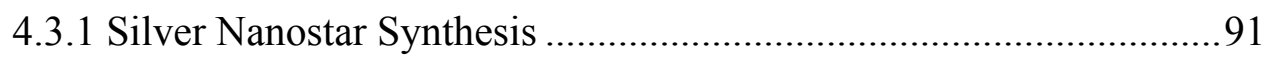

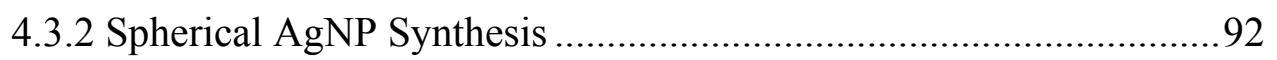

4.3.3 SERS and SEROA of Cytochrome C with AgNS .........................93

4.3.4 SERS and SEROA of Cytochrome C with Spherical AgNPs...........95

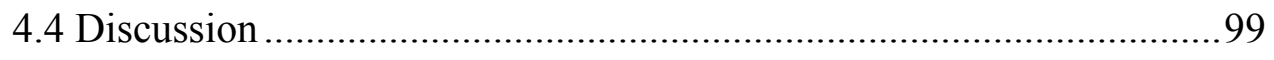

Chapter 5 Light and Temperature Sensitive Polymers for Modulated

Surface Enhanced Raman Scattering ..............................................................102

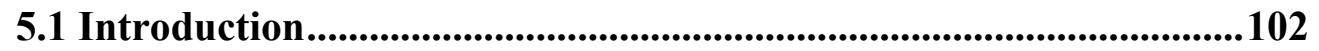

5.2 Chemical Synthesis .................................................................................104

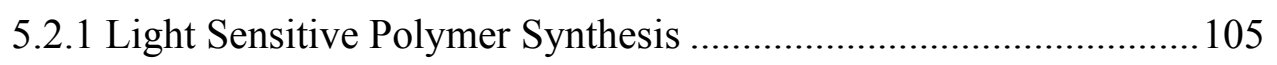

5.2.2 Light Sensitive Polymer Results and Discussion........................... 106 
5.2.3 Temperature Sensitive Polymer

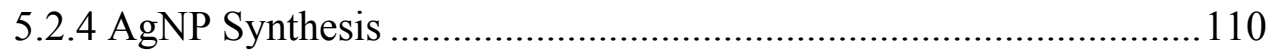

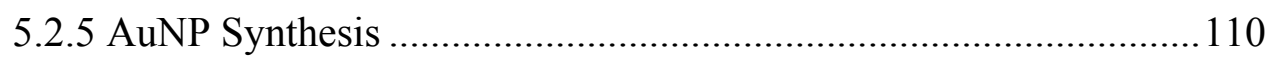

5.2.6 Conjugation of polyNIPAM with Nanoparticles .......................... 112

5.3 Modulated SERS in Colloid ..........................................................115

5.4 AgNP Immobilization on Glass Substrate and Flow Cell

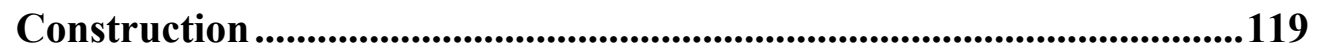

5.5 Modulated SERS on Immobilized Nanoparticle Surface..................121

5.6 Discussion and Conclusion ...........................................................126

Chapter 6 Conclusions and Future Directions...............................................130

6.1 Conclusions.........................................................................................130

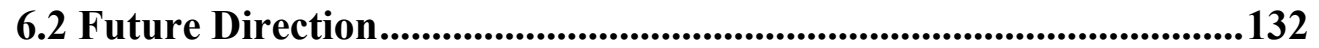

Author's Publications .................................................................................136

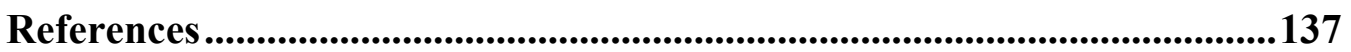




\section{Summary}

This dissertation presents three major studies utilizing Raman spectroscopy (RS) for the detection and quantification of proteins. All studies were aimed at achieving fast, minimally invasive and cost effective protein detection to potentially complement and eventually replace current methods, such as Western blotting and ELISA, which are labor intensive and slow. Our techniques may have significant effects on biomedical research, particularly with tissue bioreactors, where there is urgent demand for new and fast protein detection technology. In this work, polarized Raman spectroscopy, Raman Optical Activity, and modulated Surface Enhanced Raman Scattering (SERS) were explored.

A combination of the expanded set of spectral information provided by polarized Raman spectroscopy (PRS) that is otherwise unavailable in conventional RS was, to our knowledge, explored to enhance quantitative accuracy and robustness of protein quantification in a Raman based platform for the first time. A mixture containing two proteins, lysozyme and $\alpha$-amylase, was used as a model system to demonstrate enhanced quantitative accuracy and robustness of selective protein quantification using PRS. Concentrations of lysozyme and $\alpha$-amylase in mixtures were estimated using data obtained from both traditional RS and PRS. A new method was developed to select highly sensitive peaks for accurate concentration estimation to take advantage of additional spectra offered by PRS. The root-mean squared errors (RMSE) of estimation using traditional RS and PRS were compared. A drastic improvement in RMSE was observed from traditional RS to PRS where the 
RMSEs of $\alpha$-amylase and lysozyme concentrations decreased by 11 times and 7 times, respectively. Therefore, this technique is a successful demonstration in achieving greater accuracy and reproducibility in the estimation of protein concentration in a mixture, and it could play a significant role in future multiplexed protein quantification platforms.

The next logical direction led to Raman Optical Activity (ROA) where circularly polarized (CP) incident and scattered Raman light was used. On the whole, proteins are chemically identical, and Raman spectra, which are chemically dependent, in turn show significant overlap among protein species making deconvolution difficult and impractical. To overcome this obstacle, attention must be shifted to structure, which is a defining property that typifies a protein's role and behavior in living systems. Surface enhanced Raman Optical Activity (SEROA) is potentially well suited for this application as it can access structural information to enable us to identify individual protein species within mixtures qualitatively and quantitatively within minutes as opposed to several hours or days as required by state-of-the-art methods already mentioned. Due to the inherent weakness of ROA signals, construction of an ROA optical setup requires exceptionally precise alignment and compensatory optics to minimize non-CP Raman signals that can easily overwhelm ROA. Despite immense challenges, a crudely working ROA setup was constructed and shown to successfully generate an ROA spectrum of $(+)-\alpha$-pinene, a compound commonly used as a validation standard. 
The third study explored modulated SERS using both photo- and thermoresponsive linear polymers to vary the distance between a target protein and a surface plasmon. The modulated signal can be easily distinguished against a background of other proteins emitting a steady signal. Synthesis of a photoresponsive polymer containing azobenzene, a photosensitive compound, a biotin end group for specific recognition of avidin, and a thiol group for strong adsorption to gold or silver nanoparticles was attempted. Under visible light, the polymer collapses due to increased hydrophobicity and reduces the distance between the nanoparticles and protein molecules. Under UV light, the polymer regains its solubility and re-extends to its original random-coil conformation. The same concept holds for the thermoresponsive polymer, polyNIPAM, but only in response to temperature where it is hydrophobic and hydrophilic above and below a particular temperature, respectively. This approach was extensively tested on colloidal gold and silver nanoparticles as well as silver nanoparticles immobilized on a glass surface.

Our contribution worked towards meeting the growing demand for advanced protein detection platforms by means of polarized Raman spectroscopy, Raman Optical Activity, and modulated SERS. To date, the work presented here represents the first attempts of applying these techniques towards multiplexed protein detection and quantification. As such, it represents an expansion of these Raman techniques into biomedical diagnostics. PRS was demonstrated to deconvolute a binary protein mixture with enhanced quantitative abilities, which may play an important role in future detection schemes. ROA possesses great promise in extending Raman spectroscopy to more complex mixtures 
found in biological systems. Finally, modulated SERS, although workable in theory, was found impractical using polymers, which has refocused our attention on more structurally robust molecular constructs such as molecular motors and nano-structured plasmonic surfaces. 


\section{Chapter 1 Background}

\subsection{Raman Spectroscopy}

Raman Spectroscopy (RS) is a light scattering phenomenon from which unique spectra of molecules are obtainable. An analysis of spectra gleans intricate chemical information on a molecular level that is invaluable in a variety of applications within biomedical diagnostics. It hails enormous potential and practical utility as it is favorably non-invasive and non-destructive that makes it well suited for advancing diagnostics in the biomedical realm.

Irradiation of a sample by an intense laser beam comprised of photons each of energy $v_{0}$ results in scattered light of two varieties: Rayleigh and Raman. The first type of scattering is relatively intense and has the same frequency as the excitation radiation, $v_{0}$. Although strong, Rayleigh scattering contains little to no useful information about the analyte. Conversely, Raman scattering is comparatively weaker, around $10^{-5}$ times that of the incident beam, and possesses frequencies of the form $v_{0} \pm v_{\mathrm{m}}$ where $v_{\mathrm{m}}$ is a vibrational frequency of a molecule. ${ }^{1-3}$ The resulting bands arising from various vibrational modes are combined to construct Raman spectra that, as a whole, serve as chemical fingerprints of molecules. Bands residing at higher frequencies than the excitation radiation, $v_{0}+v_{\mathrm{m}}$, are known as anti-Stokes lines while those residing at lower frequencies, $v_{0}-v_{\mathrm{m}}$, are Stokes lines, which are significantly stronger in intensity and therefore used prevalently in Raman studies. Each band corresponds to a unique vibrational mode that depends upon the mass of the involved atoms and the force constants of their chemical bonds, which is influenced by their local chemical environment. ${ }^{4}$ From this, it can be easily recognized how the Raman spectrum is a reflection of chemical composition. 
Figure 1.1 illustrates the transition between vibrational energy levels after an incoming photon inelastically scatters upon interacting with a molecule. In the Stokes case, Figure 1.1a, the molecule transitions from the ground state $\left(\mathrm{S}_{0}\right)$ to an excited vibrational state $\left(\mathrm{S}_{1}\right)$, and the energy required for this transition is drained from the excitation photon resulting in the second scattered photon having less energy. The emergence of anti-Stokes lines, Figure 1.1b, requires the molecule to be initially in the excited vibrational state whereupon the excitation photon gains energy after returning to the ground state. At room and body temperature, as is relevant in this context, molecules rarely exist above their ground vibrational state, which is why anti-Stokes bands are significantly weaker.

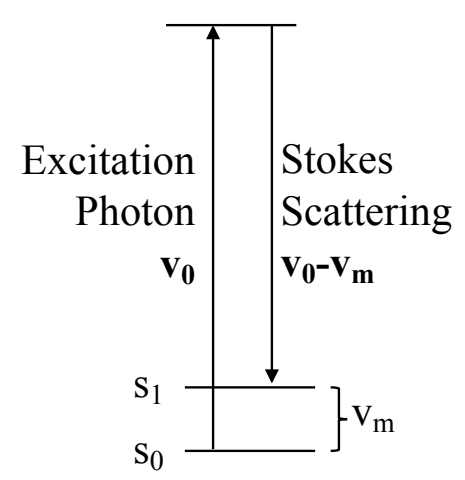

(a)

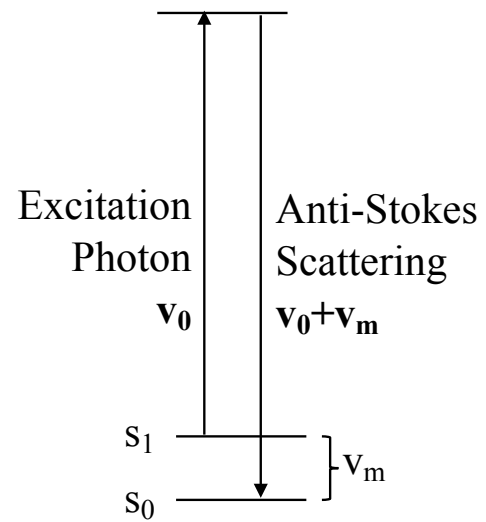

(b)

Figure 1.1 Energy level diagram of Raman scattering: (a) Stokes Raman scattering and (b) antiStokes Raman scattering. The transition energy between the ground $\left(\mathrm{S}_{0}\right)$ and the vibrational state $\left(\mathrm{S}_{1}\right)$ is denoted as $\mathrm{v}_{\mathrm{m}}$ that is in units of frequency.

\subsubsection{Strengths and Weaknesses}

Raman spectroscopy is nearly immune to interference from water and usually has no invasive and destructive requirements. Such advantages enable biological samples to be probed in vivo without extensive sample preparation using foreign solvents or destructive fixation procedures. Although these advantages are substantial, the drawbacks are equally significant. The first 
major obstacle is inherently weak signals of biological samples, ergo requiring damagingly high laser power and/or long exposure times. However, numerous signal enhancing techniques such as Surface Enhanced Raman Scattering (SERS), which will be discussed later, can effectively overcome this obstacle. Another major drawback is strong fluorescence emitted by molecules that can mask Raman signals. Using lasers with lower energies near the infrared range (750-1064 nm) markedly reduces fluorescent intensity, but at the expense of sensitivity given the intensity of Raman scattered radiation is inversely proportional to the fourth power of the excitation wavelength $\left(I \propto 1 / \lambda^{4}\right){ }^{3-5}$ In general, the end user must decide whether reduced fluorescence or increased sensitivity is more suitable for the application at hand.

The most commonly used fluorescence removal methods are polynomial baseline fitting, wavelet transform, and derivative based techniques. ${ }^{136}$ These techniques vary in effectiveness. Polynomial methods rely heavily on user experience and can be relatively slow when fitting high order polynomials. Additionally, they suffer from poor performance in datasets with a low signalto-noise ratio. The remaining two methods are known to distort the original spectra in terms of peak shape. The studies discussed in this thesis used an improved wavelet-based background subtraction method, developed by Zhang et al. ${ }^{136}$, that greatly minimized spectral distortion in comparison to previous wavelet methods.

\subsection{Polarized Raman Spectroscopy}

Polarized Raman spectroscopy (PRS) measures the intensity of scattered light parallel $\left(I_{\|}\right)$and perpendicular $\left(I_{\perp}\right)$ to the electric vector of the incident

radiation. ${ }^{1}$ It should be explicitly noted that Raman bands are sensitive to the 
linear polarization (LP) state of both the incoming-excitation and outgoingscattered radiation as well as the scattering angle. PRS is sensitive to the orientation and position of crystalline samples, and used primarily for structural analysis. It also distinguishes between symmetric and asymmetric molecular vibrations in a situation where all molecules are freely oriented as with liquids or gases. In a $180^{\circ}$ backscattering configuration using linearly polarized incident radiation, $I_{\|}$and $I_{\perp}$ originate from symmetric and asymmetric vibrations, respectively. ${ }^{1-3}$ Measuring these intensities is accomplished by rotation of linear polarizers placed in the excitation and scattering pathways. The depolarization ratio is defined as the ratio between the intensity of perpendicular and parallel scattered Raman light, which is commonly used to classify symmetric and asymmetric vibrations.

\subsection{Raman Optical Activity}

Raman optical activity (ROA) is a technique that enables one to observe minute differences between the intensity of right and left circularly polarized (RCP and LCP) light that is incident on and/or scattered by chiral molecules. ${ }^{6-8}$ This generates bisignate (having positive and negative intensities) spectra that provide invaluable stereochemical information and is especially useful for elucidating the structure of complex biopolymers such as proteins and other biomolecules. ${ }^{8-12}$ Unlike conventional Raman spectroscopy, in which spectral bands largely depend on chemical composition, primarily arising from amino acid side chains, that causes band overlapping of nearly all bands, ROA bands result from key structural motifs arising from the secondary and tertiary structure. $^{6,9}$ The weak scattering by water molecules affords this technique a powerful and unique advantage of probing biomolecules in their native conformation. ROA bands are directly assignable to secondary elements such as 
$\alpha$-helices and $\beta$-sheets as well as to loops and turns that link these components together, and allow the overall fold of a protein to be characterized. ${ }^{13-15}$

\subsubsection{Forms of ROA}

There exist four forms of ROA, which are named the following: incident circular polarization (ICP-ROA), scattered circular polarization (SCP-ROA), and dual circular polarization (DCP-ROA) which is comprised of two types, namely $\mathrm{DCP}_{\mathrm{I}}-\mathrm{ROA}$ and $\mathrm{DCP}_{\mathrm{II}}-\mathrm{ROA}$. The first three forms produce qualitatively identical information and possess highly similar spectra while reports of $\mathrm{DCP}_{\mathrm{II}}-\mathrm{ROA}$ are scant, and reveal this method to provide a mainly flat spectrum with little information. ${ }^{6,16,17}$ Each form of ROA is defined as the following. ICP-ROA uses incident circularly polarization radiation while detecting unpolarized scattered light, SCP-ROA uses linearly or unpolarized incident radiation while detecting both right and left handed circularly polarized light, and in DCP-ROA both the incident and scattered light are circularly polarized such that the incident and scattered light are in-phase in $\mathrm{DCP}_{\mathrm{I}}$ and out-of-phase in $\mathrm{DCP}_{\mathrm{II}}$. Please refer to Figure 1.2 for an energy-level diagram of each ROA type for a more intuitive representation. The intensity of scattered radiation, $I$, from this point forward will contain a super- and subscript indicating the excitation polarization and scattered polarization states, respectively. For example, unpolarized excitation radiation and right handed scattered light is written as $I_{R}^{U}$. Given this convention, it is simpler to write each form of ROA as shown in Table 1.1. The conventional Raman spectrum, referred to as the parent Raman spectrum, is simply the sum of each polarized spectrum. 
Table 1.1 Mathematical representation of all forms of ROA and associated parent Raman quantities

$\begin{array}{ccc} & \text { ROA } & \text { Parent Raman } \\ \text { ICP-ROA } & I_{U}^{R}-I_{U}^{L} & I_{U}^{R}+I_{U}^{L} \\ \text { SCP-ROA } & I_{R}^{U}-I_{L}^{U} & I_{R}^{U}+I_{L}^{U} \\ \text { DCP }_{\text {I }} \text { ROA } & I_{R}^{R}-I_{L}^{L} & I_{R}^{R}+I_{L}^{L} \\ \text { DPC }_{\text {II }} \text { ROA } & I_{L}^{R}-I_{R}^{L} & I_{L}^{R}+I_{R}^{L}\end{array}$
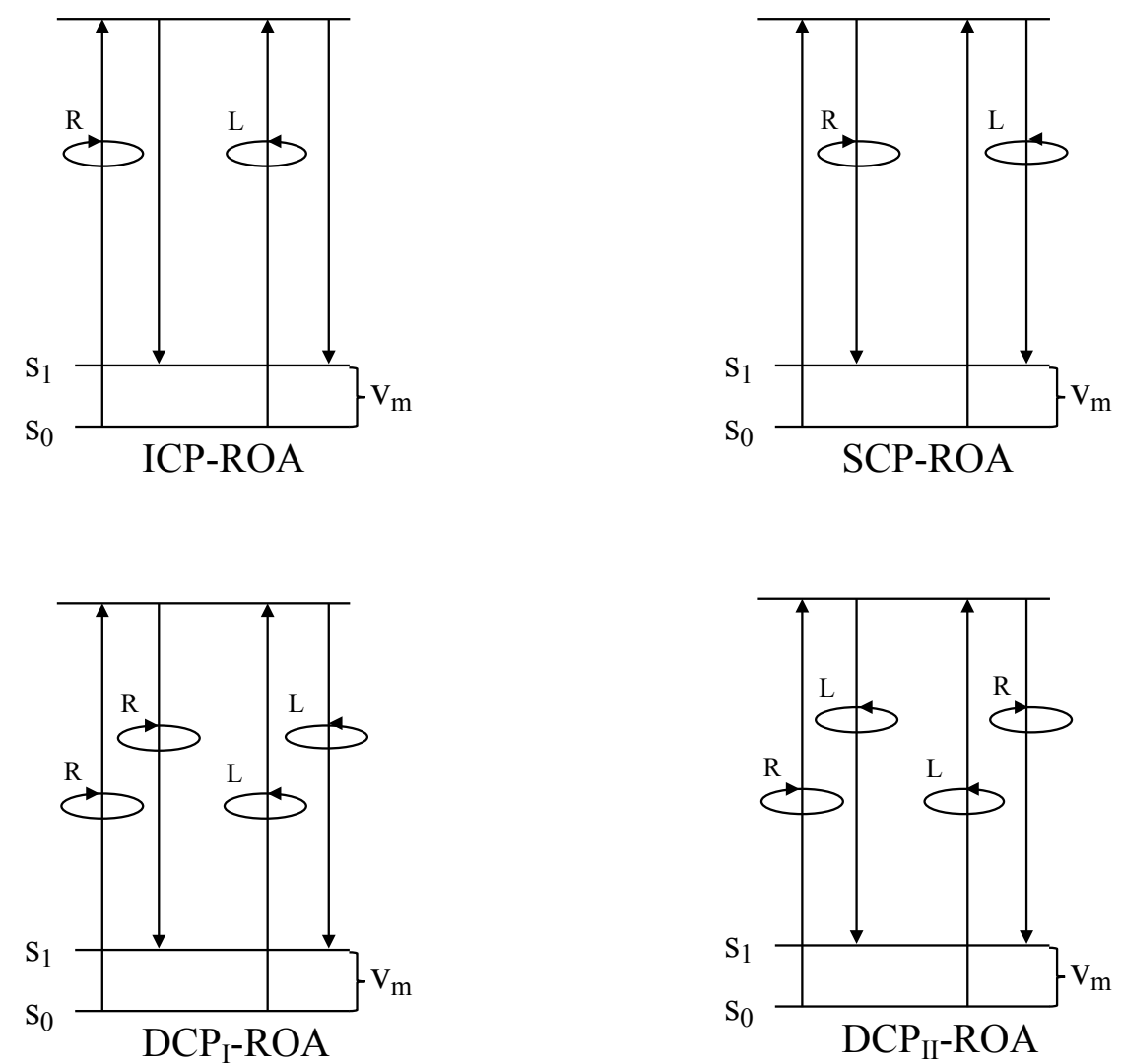

Figure 1.2 Energy level diagrams of all four forms of Raman Optical Activity (ROA)

\subsubsection{Drawbacks of ROA}

Although ROA shares the benefits of conventional Raman spectroscopy, it poses an even greater challenge due to the extreme weakness of signal strength being three to five orders of magnitude smaller than that of parent Raman bands. ${ }^{18-21}$ Due to uncertainty inherent to all forms of spectroscopy, one primary type of noise, known as shot noise, is the square root of signal intensity. ${ }^{4}$ Consequently, around $10^{10}$ Raman counts are required to clearly 
reveal the weakest ROA signals, which are $10^{-5}$ times smaller than the parent Raman intensity. Therefore, the noise level, being $10^{5}$ counts after taking the square root, must be at least five orders of magnitude smaller than the parent Raman bands amounting to a signal-to-noise ratio of at least 1 or greater. ${ }^{6,22}$ Note that noise is inclusive of fluorescence and stray light. This calls for impractically long exposure times on the order of many hours and high laser power close to $1 \mathrm{~W}^{22,23}$ Given this drawback, excitation radiation within the blue end of the spectrum is favorable when considering the dependence of Raman intensity on wavelength, i.e. $I \propto 1 / \lambda^{4}$, and the dependence of ROA intensity on wavelength, i.e. $I \propto 1 / \lambda^{5} .^{22,23}$ However, biological samples typically produce significant fluorescence that demand the use of red excitation. Unfortunately, this causes severe reduction in ROA sensitivity. As a compromise, green excitation is the most suitable choice and most prevalently used. $^{6,22,23}$

The circular intensity difference (CID) is commonly calculated for each suspected ROA band where, for a given excitation beam of polarization $\mathrm{x}$, $\mathrm{CID}=\left(I_{R}^{x}-I_{L}^{x}\right) /\left(I_{R}^{x}+I_{L}^{x}\right)$. A typical ROA peak will have an intensity on the order of $10^{-3}$ to $10^{-5}$ of the parent Raman signal. Signals with a CID outside this range are not a result of the sample's chirality, but originate from imperfections inherent to the optics used.

\subsubsection{Spectral Artifacts}

The most serious problem in ROA measurements is spectral artifacts ${ }^{6,24-26}$ that generate artificial differences between left and right circularly polarized spectra that are not due to the chiroptical properties of the analyte. ${ }^{27}$ The cause arises from imperfections in the polarization optics, linear polarization components in 
the circularly polarized (CP) incident laser beam (and vice versa), residual birefringence in optical components, and refraction occurring at angled surfaces such as lenses and the wall of the sample cell. ${ }^{27}$ Any presence of LP components in $\mathrm{CP}$ radiation reaching or emitted by the sample is essentially elliptical that leads to depolarization ratio related artifacts given the sensitivity of Raman scattering to orthogonal LP states as touched on earlier. ${ }^{6}$ Implementation of highly customized optics can significantly suppress artifacts, which include fast rotating polarization conditioning optics rotating at 13,000 $\mathrm{rpm}^{28}$ and, in this study, half- and quarter-wave plates that require fine rotational adjustment with a precision of $0.05^{\circ} \cdot{ }^{25}$ Another principle source of artifacts is an imbalance in the RCP and LCP components present in the excitation and/or scattered radiation leading to an excess of parent Raman signals leftover after subtraction of both CP states. ${ }^{6}$

\subsection{Surface Enhanced Raman Scattering (SERS)}

Molecules brought within close proximity of metal substrates results in unprecedented enhancement of Raman signals of more than six orders of magnitude. ${ }^{4,29,30}$ Silver and gold are predominantly used since they are known to generate the greatest enhancement, however, many other noble metals such as copper and platinum also support SERS, but less effectively. ${ }^{31}$ The magnitude of enhancement is a function of the metal type, morphology, particle size (in colloidal systems), and roughness. High enhancement is observed with metals of high reflectivity and also depends on laser wavelength and the analyte's adsorption characteristics with the SERS surface. ${ }^{4}$ 


\subsubsection{SERS Mechanism}

There exist two independent mechanisms giving rise to the SERS effect, namely: chemical and electromagnetic enhancement. The former only accounts for enhancements of up to approximately 100 times $^{4}$, and involves chemisorption of the molecule with the metal surface. ${ }^{32}$ As a result, the electronic state of the analyte may shift and/or new resonant electronic states may emerge due to a newly formed chemical bond with the metal.

As for the latter mechanism, it contributes the most enhancement and is directly attributed to localized surface plasmon resonances (LSPRs) induced by an incoming electromagnetic field (i.e. light). ${ }^{33}$ Free electrons of metallic nanoparticles exposed to an electromagnetic (EM) field experience force that causes them to concertedly oscillate in phase with the frequency of the external EM field, ergo forming surface plasmons. As a result, these surface plasmons generate their own EM field which, when combined with that of the incoming EM field, amplifies Raman signals of nearby molecules. Excitation of plasmon resonance is most efficient when the laser source is tuned to the LSPR band. Light at wavelengths outside this band are merely scattered or transmitted as their energy is not used to excite surface plasmons. Gold nanoparticles, for example, around $30 \mathrm{~nm}$ in diameter absorb light in the visible blue-green range of the spectrum $(\sim 520 \mathrm{~nm})$, where surface plasmons are excited, whereas light in the red range $(\sim 700 \mathrm{~nm})$ is merely scattered creating the distinctive red color of gold colloidal solutions. Increasing NP size causes a redshift in the LSPR band causing gold colloids to become bluer or more purple.

Intuitively, the enhancement degrades as the distance between the analyte and metal surface increases characterized by $[r /(r+d)]^{12}, r$ being the metal 
particle's radius and $d$ being the distance of the analyte from the surface of the NP. ${ }^{32,34}$ Hence, maximum enhancement occurs when in direct contact with the surface, and larger NP diameter increases the overall enhancement.

\subsubsection{Synthesis and Characterization}

SERS substrates can be prepared in variety of morphologies; a common shape is spherical nanoparticles (NPs) in a colloidal suspension. A typical synthesis procedure begins with metal cations that undergo reduction by a reducing agent, such as hydroxylamine or sodium borohydride, in the presence of a surfactant or other stabilizers, such as polyacrylic acid or citrate. Once reduced, neutralized metal atoms begin to self-assemble in a process known as nucleation to form nanoparticles. Finally, surfactants provide nanoparticles with charge to attain stability in the aqueous phase that would otherwise induce irreversible flocculation.

Routine inspection of the size, shape, aggregation state, and approximate concentration of NPs is done using UV-VIS absorption spectroscopy that can reveal the LSPR peak location and height for estimating these properties. Dynamic Light Scattering (DLS) is also a convenient method for ascertaining the size, polydispersity, and aggregation of NPs in solution. Although these methods only provide approximate values, they are of sufficient accuracy for routine use. The most accurate measurement of size, shape, and polydispersity is provided by transmission electron microscopy (TEM). ${ }^{35}$ However, this method cannot assess the aggregation state nor perform real-time monitoring of NP size. Lastly, sample preparation is tedious, and TEM is impractical for routine measurement of particle size, generally speaking. 


\subsubsection{SERS of Proteins}

With regard to SERS of proteins, metal NP colloids, often AgNPs or AuNPs, are combined with proteins in solution. In the common case of negatively charged NPs, which typically result from a monolayer of surface bound citrate anions or strongly bound halides, cationic proteins can bind by electrostatic attraction and induce the formation of NP/protein aggregates. Correspondingly, anionic or neutral proteins possess less propensity to induce aggregation ${ }^{36}$, in which case an aggregation agent, typically $\mathrm{NaCl}, \mathrm{MgSO}_{4}$, and $\mathrm{Na}_{2} \mathrm{SO}_{4}$, sometimes called a flocculent, is introduced. The major importance of this occurrence is the emergence of a new plasmon resonance peak caused by newly formed aggregates of larger size and irregular shape. The position of this new resonance peak is red-shifted relative to the LSPR band of the original colloid and is essential for the observance of intense surface enhancement that cannot be achieved by the LSPR of single colloidal nanoparticles. ${ }^{37-40}$ This is due to the electromagnetic coupling effect when two or more nanoparticles come into close proximity to one another giving rise to a dramatic increase in SERS intensity. ${ }^{41-43}$ Given the sparse concentration common in a typical colloidal synthesis of nanoparticles, within the nanomolar range, aggregation is essential for obtaining detectable SERS signals from molecules in solution not only due to the intensification of the local EM field, but also that aggregates are visible under a microscope that can be conveniently found when searching for areas of high EM field enhancement (hot-spots). The closer the excitation wavelength and resonance band (after flocculation) coincide, the more intense the enhancement.

Aggregates are highly unstable and usually settle out of solution after about 30 minutes. In some cases, this is beneficial for easily finding locations for 
interrogation at the bottom of the sample chamber. However, in vertically oriented cuvette-based arrangements, it is desirable for these aggregates to remain suspended in solution since the laser spot can only be focused at a midheight location within the cuvette. Moreover, the random motion of aggregates creates a constantly changing baseline making the spectra of two polarizations incomparable and unfit for subtraction to obtain a Surface Enhanced Raman Optical Activity (SEROA) spectrum. By far the most serious disadvantage that is commonly known is lack of reproducibility of the same analyte both quantitatively and qualitatively due to the dependence on varying protein adsorption orientations, laser-induced reactions, surface chemistry of NPs, and location of interrogation. ${ }^{6,36,44}$ Fortunately, excellent reproducibility has been achieved in aqueous solution to reduce laser-induced effects ${ }^{45}$ and by adsorbing a monolayer of halides on the surface of nanoparticles to shield against chemical interaction with the protein that may incite denaturation. ${ }^{36}$ More details of addressing this issue are provided in Chapter 4. 


\section{Chapter 2 Raman Spectroscopy Based Techniques in Tissue Engineering - An Overview}

Significant portions of this chapter have been taken directly from a previously published paper entitled "Raman Spectroscopy Based Techniques in Tissue Engineering - An Overview", in which the first author is the author of this dissertation. The remaining two authors, listed here in the appropriate order, Dr. Quan Liu and Dr. Mayasari Lim, are hereby acknowledged for their valued contribution.

\subsection{Introduction}

The ever increasing clinical demand for replacement tissue and cells has led to a rapid expansion of research capabilities in the field of tissue engineering and regenerative medicine (TERM) as these areas possess the strongest potential for treating debilitating and mortal illnesses that cannot otherwise be remedied. ${ }^{46}$ As a result, tissue bioprocessing methods are rapidly advancing with novel bioreactor and biomaterial designs, but cell monitoring technologies are still largely cumbersome and lagging.

Monitoring tissue growth in cell culture still remains a major challenge despite current analytical techniques that allow us to probe many factors such as RNA expression, intracellular/extracellular protein expression, and exogenous components. Current methods including enzyme-linked immunosorbent assay (ELISA), polymerase chain reaction (PCR), and western blotting (followed by mass spectrometry) are inefficient, invasive, and destructive. ${ }^{47}$ Placzek et al. ${ }^{48}$ claims that deconvolution of molecular signals to facilitate bioprocess monitoring control measures and automation are among the essential design 
principles that will lead to proper end-product functionality and integration. These components are integral to creating clinical products derived from stem cell bioprocessing. Present limitations clearly preclude us from fully realizing these principles in a reliably streamlined, hassle free manner and therefore obstruct TERM from becoming established in clinical practice. Furthermore, at the forefront of tissue engineering research, the assessment of cells/tissues grown in three-dimensional (3D) scaffolds require biological information that goes beneath the surfaces of the scaffold without disassembling the overall tissue construct; this remains a challenge yet to be adequately addressed.

Raman spectroscopy (RS) is a noninvasive, nondestructive, and label-free technique that can complement current methods and potentially replace them as a real-time monitoring platform. The application of RS in biology and medicine has steadily increased in recent decades and has shown growing promise to eliminate the need for exogenous molecular markers and other invasive/destructive methods. Although the applications and methods of RS for tissue engineering have been addressed in previous reviews ${ }^{49-51}$, we put special focus on the detection of proteins as it is one of the major endpoint indicators for any biological tissue. Moreover, in light of recent advances in Raman enhancement methods and techniques, we present an updated account of RS for monitoring cells and scaffolds.

\subsection{Background}

\subsubsection{Monitoring process components in bioreactors}

Implementation of 3D scaffolds to engineer cells into tissue constructs is an approach aimed at achieving both structural and functional integrity of engineered tissues for clinical use. Under these conditions, cells are predisposed 
to respond and differentiate according to mechanical and chemical cues spatially situated in their immediate surroundings. ${ }^{52}$ However, interrogating cells within their 3D space while they are in culture can be rather challenging. This requires the detection of cells, proteins, and scaffold material to provide sufficient information for evaluating not only the cellular components but also the integrity of the tissue engineered construct. Current commercial platforms in bioreactor monitoring provide only the standard physiological parameters such as $\mathrm{pH}$, oxygen, electrolytes, and nutrients/metabolites, and do not go beyond to provide relevant biological information at the level of nucleic acids, proteins, or lipids. The analysis of relevant biological information pertaining to its genetic or functional level rely heavily on traditional methods in molecular biology such as PCR and protein gel electrophoresis, which have destructive requirements that make them unsuitable for online monitoring, but are still routinely used for endpoint analysis to evaluate tissue products. Other impediments pertaining to the use of such techniques for monitoring include prolonged procedures, high probability for error subject to skill level, and expensive reagents. The current lack of a rapid and non-destructive technology for bioreactor monitoring impedes progress in the control of large-scale processes in bioreactors and bio-scaffold design optimization for many tissue engineering applications. There is a significant gap in monitoring technologies to facilitate progress in ensuring the quality and standards required for clinical manufacturing of engineered tissues. Given the context of current limitations in monitoring techniques, this chapter will focus on the detection of cells, scaffolds, and proteins in terms of how Raman spectroscopy can be used to detect these important biological process components. 


\subsubsection{Role of RS for online monitoring in TERM}

Online monitoring of intracellular status as well as extracellular protein levels remains unrealized in bioreactor systems. Raman spectroscopy can provide quantitative chemical analysis at the cell, protein, and even DNA level that can potentially be performed in real time with the utilization of previously mentioned enhancement methods. Using biochemical data to assess cellular and differentiation status would make it possible to avoid invasive visualization techniques that are highly inefficient and semi-quantitative. Raman spectroscopy has the unique advantage to penetrate beneath the surface of transparent and semi-transparent materials making it possible for nondestructive and noninvasive sub-surface evaluation of 3D scaffolds. Protein detection schemes would be greatly expedited as complicated gel separation and quantification procedures could be circumvented. If successful at achieving these objectives, TERM will gain the needed advances in bioprocessing technology to accelerate cell based therapies.

\subsection{Applications of Raman spectroscopy to monitor target bioreactor} components

In this section, various techniques based on $\mathrm{RS}$ for monitoring the key biological components in bioreactors, including cells (section 2.3.1), scaffolds (section 2.3.2) and proteins (section 2.3.3), are reviewed. A special emphasis is made on protein detection due to the distinctive importance of proteins in TERM and the extensiveness of protein detection studies conducted using RS thus far. 


\subsubsection{Monitoring Cells}

In the context of tissue engineering, monitoring biological properties of cells and/or its associated changes provides important information regarding cell status such as viability and functional state of the cell. The intracellular components of a cell, which includes the cell nucleus, organelles and cytoskeleton, contain important biological information that can be extracted from nucleic acids, proteins, lipids, and carbohydrates. For example, detecting the expression of specific functional proteins provides an indicator for the functional status of a cell, and is highly relevant to cell differentiation and tissue engineering. Further insights into biological interactions of DNA and proteins can also be obtained from RS methods, and have been discussed in other reviews. ${ }^{53,54}$

Raman spectroscopy has been explored to measure multiple biological components from the intracellular space of a cell. Changes in the expression level or structure of nucleic acids, proteins, and lipids are highly reflective of intracellular activity and commonly used as spectral markers for classifying cell type and/or status. This has been extensively applied for the identification of biological tissue samples in various diseases and cancer. ${ }^{55}$ Sensitivity of RS techniques has reached the level of identifying subtypes within a cell line. In one example, Movasaghi et al. ${ }^{56}$ exploited spectral differences using partial least squares discriminant analysis to identify different subtypes that are either sensitive or resistant to chemotherapy in the testicular cancer cell line $833 \mathrm{k}$. This chapter clearly highlights that RS is sufficiently sensitive and can objectively differentiate characteristics within subtypes of a cell line based on differences in the chemical groups or cellular chemical compounds in response to a chemical agent. Such a technique can be useful for applications in tissue 
engineering as well by monitoring changes in the chemical or biochemical constituents of cells. Here we focus our attention to the use of RS as a means to monitor engineered tissue. Stem cell differentiation is one of the primary endpoints of interest in the context of tissue engineering.

\subsubsection{Assessing stem cell differentiation}

In the realm of stem cell analysis, Pascut et al. ${ }^{57}$ demonstrated the use of temporal and spatially resolved Raman band intensity profiles to noninvasively monitor intracellular molecular changes of embryoid bodies (EB) composed of hESCs as they differentiated into cardiomyocytes $(\mathrm{CMs})$ in vitro. In EBs that contained successfully differentiated CMs, the onset of cardiac contraction after 7 days as well as elevated levels of $\alpha$-actinin was found to coincide with a significant intensity increase of several Raman bands. In an earlier study ${ }^{58}$, the spatial imaging of hESC derived CMs revealed elevated levels of glycogen and myofibrils, which can be used as spectral markers to discriminate CMs in a heterogeneous cell population. It is well established that subjecting stem cells to NIR laser exposure under a certain threshold for Raman interrogation does not reduce pluripotency of stem cells nor cause cellular damage. ${ }^{59,60}$ This is significant as it avails countless possibilities in the use of NIR technology as future online monitoring tools for bioreactor systems. As these spectral markers can be detected noninvasively and rapidly compared to lengthy immunohistochemistry techniques that can require days to complete. The potential of online Raman monitoring to help forecast cell fate rapidly in a matter of seconds or minutes during stem cell differentiation is an exciting opportunity that could advance the field of TERM. 
Direct imaging of intracellular biochemical distribution using coherent antistokes Raman scattering (CARS) was demonstrated by Downes et al. ${ }^{61}$ to rapidly identify two distinctive resultant cell types, osteoblasts and adipocytes, both differentiated from adipose-derived stem cells (ADSC). The spectra for constructing distribution maps of lipids largely indicated by the $\mathrm{C}-\mathrm{H}_{2}$ stretch at $2854 \mathrm{~cm}^{-1}$ for adipocytes and hydroxyapatite at $960 \mathrm{~cm}^{-1}$ for mineralization within osteoblasts were obtained using dwell times within the microsecond domain for each pixel. The imaging capability of this technique could potentially provide a new dimension of invaluable data for TERM diagnostics once technological advancements enables CARS to be a facile and economical method.

\subsubsection{Probing intracellular substance}

Using confocal Raman spectroscopy to directly interrogate individual cells is gaining increasing attention. Obtaining valuable biochemical information without inducing photochemical or photophysical damage remains problematic primarily due to inherently small Raman cross sections of biological analytes. In the past studies using non-resonant confocal RS, high laser power and prolonged exposure times were required to obtain sufficiently intense spectral band markers indicative of protein and DNA. ${ }^{62,63}$ This illustrates the need for faster and more efficient methods in RS for large-scale cellular evaluation in tissue engineering applications.

Although SERS NPs can greatly enhance Raman signals, introducing NPs into the intracellular space may risk endosomal/lysosomal encapsulation upon passive uptake and perturbations to normal cellular functioning. As a means to avoid endocytosis, Shamsaie et al. ${ }^{64}$ synthesized intracellularly grown gold 
nanoparticles (IGAuN) within MCF10 epithelial cells. In contrast to past SERS studies not using this technique, no peak around $500 \mathrm{~cm}^{-1}$ was detected that is indicative of the disulfide bond vibration -an abundant chemical bond within lysosomes. Evasion of cellular protection mechanisms was therefore strongly evident. Consequently, increased NP distribution throughout the cytoplasm and nucleus was observed, which provided highly resolved spectra that could be primarily attributed to nuclear and cytoplasmic materials. In another recent study by Liu et al. ${ }^{65}$, a non-toxic method for accelerated growth of IGAuN was achieved in A549, 4T1, and HeLa cells making it more practical for fast characterization (such as early cancer detection) in approximately 15 hours. Poly(vinylpyrrolidone) (PVP)-functionalized graphene oxide (GO) was used as an activation platform for gold nanostructure biosynthesis. Intracellular formation of PVP/GO/IGAuNs hybrid nanocomposites led to a uniform SERS signal distribution throughout cells' interior and provided signal enhancement that was roughly five times stronger in comparison to IGAuNs alone. The SERS spectra also revealed new bands only observable using these hybrid nanoparticles that provided additional valuable chemical information. Moreover, SERS spectra were even detected from the nucleolus as GO facilitated the delivery of PVP across the nuclear membrane.

\subsubsection{Probing Scaffolds}

Interrogation of cell/tissue development in $3 \mathrm{D}$ scaffolds can be achieved using confocal fluorescence microscopy. Despite our reliance on this method, it is an invasive technique that requires exogenous fluorophores. The reconstruction of its structural organization and tissue architecture requires complex image analysis algorithms and automation. Moreover, the interrogation of cells at greater depths beneath the scaffold surface remains challenging. Visualization 
of tissue architecture in larger constructs requires even more sophisticated imaging techniques that are currently being pursued and/or complex preparative techniques to push beyond the working distance of the objective. ${ }^{66}$

Raman spectroscopy does not necessitate fluorophores, and has great potential to non-invasively and non-destructively evaluate $3 \mathrm{D}$ biological structures and tissue engineered constructs. For such applications, the choice of biomaterial influences spectral quality and detection accuracy of RS. Thus it is paramount to select materials that do not generate high fluorescence and unwanted background peaks. Bonnier et al. ${ }^{67}$ reported collagen gels as an attractive choice for biomaterials by demonstrating its low background contribution as comparable to that of water. SERS spectra obtained from live A549 cells grown on collagen gel substrates were shown to exhibit nearly no background interference.

\subsubsection{Monitoring Scaffolds}

Evaluating biocompatibility and cell adhesion to novel scaffolding material is invaluable in tissue engineering. Fluorescence microscopy and scanning electron microscopy (SEM) are commonly used to investigate cell and scaffold architecture, but they are invasive methods and highly subject to user experience. In cartilage tissue engineering, tracking the formation of collagen type II and proteoglycans, i.e. components that make up the bulk of the extracellular matrix (ECM), is a direct way to evaluate tissue status. Jones et al. ${ }^{68}$ used Raman spectral data to monitor in situ responses of chondrocytes to 3D bioactive glass foam scaffolds by detecting and quantifying type II collagen secreted into the ECM. The steady growth of ECM was observable according to the peak intensity $\left(1667 \mathrm{~cm}^{-1}\right.$ and $1270 \mathrm{~cm}^{-1}$ indicative of collagen $)$ that 
increased over time. In situ Raman evaluation was reported by Kunstar et al. ${ }^{69}$ for micro-tissue formations in agarose-microwell arrays. These arrays are capable of continuous perfusion enabling real-time analysis of live cells and tissue formation. In this case, since the cell/tissue samples do not have to be transferred to a microscope slide for Raman analysis, tissue formation could be interrogated rapidly and noninvasively during cell culture. ECM formation was marked by the $937 \mathrm{~cm}^{-1}$ Raman band. In a more recent study by Kunstar et al. ${ }^{70}$, Raman microspectroscopy and fiber-optic Raman spectroscopy were used to monitor ECM formation of primary bovine chondrocytes deposited into $3 \mathrm{D}$ poly(ethylene oxide terephthalate)-poly(butylene terephthalate) (PEOT/PBT) scaffolds. Raman bands $937 \mathrm{~cm}^{-1}$ and $1062 \mathrm{~cm}^{-1}$ attributed to collagen and proteoglycans, respectively, were chosen as spectral markers. Most notably, the use of fiber-optic probes facilitated simultaneous acquisition of Raman signals by placing probes in various pores within the scaffold. This generated an overall averaged ECM Raman signal to provide insight into ECM formation in the entire tissue.

Nandagwali et al. ${ }^{71}$ reported that degradation characteristics of polymerscaffolds are critical for the development of temporary tissue replacements. The rate at which polymer-scaffolds degrade should decompose at a rate coinciding with the natural regenerative processes of the body. The degradation characteristics also affect the ultimate quality of newly formed tissues. The most accurate characterization must be obtained in vivo to avoid perturbing disassembly procedures required by in vitro assays. In this study, RS was used to identify different chemical species in the biodegradable structure using the multivariate K-means data clustering method. The classification accuracy for different components was reported as high as $95 \%$. Bioactive glass is a strong 
candidate for making novel $3 \mathrm{D}$ porous scaffolds and was investigated by Moimas et al. ${ }^{72}$ using RS and SEM. The effect of scaffold degradation with respect to soaking time in simulated body fluid and glass composition was the core focus in this study. It was revealed that the ratio of network modifiers to the silica structure can be tuned to modulate degradation properties of the scaffold. van Apeldoorn et al. ${ }^{73}$ studied degradation of poly(lactic-co-glycolic acid) after macrophage phagocytosis by monitoring changes in Raman band intensities to evaluate immune rejection induced by macrophages to foreign scaffold material.

\subsubsection{Evaluating Surface Chemical Properties}

Optimization of surface properties is imperative for the success of any scaffolding material to direct cell attachment and/or maximize specific protein adsorption. Surface modification is a field of intense study that aims to modify the chemical and physical properties of a scaffold's surface to enhance biocompatibility. Although the studies cited here had destructive sample requirements due to the use of both commercial and homemade Raman microscopes, they are still worth mentioning because alternative optical geometries, such as the fiber-optic probes discussed earlier, could later be implemented to obtain the same Raman spectra non-destructively.

Luk et al. $^{74}$ characterized the modification of polycaprolactone to possess carboxylic acid groups on the surface by means of gamma irradiation-induced grafting. Carboxylic acid groups are commonly used to decrease hydrophobicity and covalently bind biomolecules. Amine and phosphate groups were also reported as effective moieties that have enhanced cell attachment and growth. Raman analysis was used to evaluate the penetration 
depth achieved by the grafting process under varying parameters by observing the band area ratio between the newly formed band at $1680 \mathrm{~cm}^{-1}$ corresponding to the double bond of a carboxylic acid group and the band at $1730 \mathrm{~cm}^{-1}$ corresponding to ester groups. A study by Rim et al. ${ }^{75}$ attempted to control stem cell fate by producing poly(L-lactide) (PLLA) fibers coated with polydopamine (PD-PLLA). Chemical composition of the surface was confirmed in a non-destructive fashion using RS when new peaks emerged at $1335 \mathrm{~cm}^{-1}$ and $1568 \mathrm{~cm}^{-1}$ that correspond to the aromatic groups of polydopamine. Jain et al. ${ }^{76}$ used RS to compare the effect of ultraviolet and oxy-plasma (OP) treatment of amorphous carbon structures and assessed the response of attached neuronal cells. After identifying Raman spectral bands at $1360 \mathrm{~cm}^{-1}$ and $1580 \mathrm{~cm}^{-1}$ that are associated with the symmetrical characteristics of a graphitic lattice, OP treatment was found to increase structural disorder and decrease crystallinity of the carbon structures.

\subsubsection{Detecting Proteins}

In the remaining section, the selection criteria of the best detection modality will no longer hinge upon non-invasive and 3D interrogative requirements, but rather on the ability to extract chemical information. Intracellular and extracellular proteins can provide biological, structural, and functional information of a tissue engineered construct. In the extracellular space, proteins secreted by cells can indicate the biological function of a specific cell type or mediate the balance of biological factors that may influence the fate of other types of surrounding cells. The presence of proteins solvated within a bioreactor system is crucial for the successful development of any engineered tissue. Exogenous factors such as growth factors and cytokines provide biochemical cues that are important for inducing cells in vitro and essential for 
directing stem cell differentiation and ensuring proper cell fate. Proteins may also be secreted by cells as they mature and can serve as a positive indicator of final cell type. Determination of protein concentration at various time points is highly valuable for assessing bioreactor status in terms of protein depletion and secretion rates. In particular, exogenous proteins such as growth factors are highly purified and costly, making it important to optimize the amount added to ensure complete differentiation among cells while minimizing costs. Although ELISA is highly sensitive and quantitatively precise, it is extremely labor intensive and costly. Western blotting can provide only semi-quantitative information and suffers from cross reactivity due to secondary antibody binding to non-target proteins. Both methods are cumbersome and time consuming. The use of RS can potentially avoid these disadvantages and contribute to a fast and cheap detection platform to quickly obtain feedback for regulating input and output parameters.

Characterization of proteins using RS is achievable via extrinsic or intrinsic methods. The former employs Raman reporter molecules that generate a minimal number of tall and narrow peaks allowing for easy identification and greater multiplexing potential. In this approach, multiple markers are detectable using only one excitation wavelength. Such reporters overcome the limitations of traditional fluorescence methods in photobleaching and overlapping broad peaks. Despite this fact, some fluorescent probes (tetramethylrhodamine isothiocyanate and atto610) ${ }^{77}$ as well as staining dyes

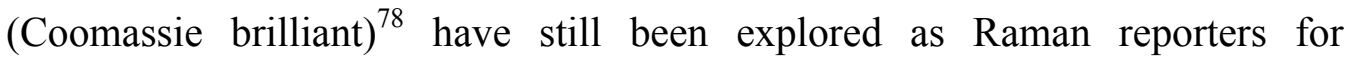
investigating protein-protein and protein-ligand interaction as a potential application in protein detection. As extrinsic techniques typically link reporter molecules with large Raman cross sections to metal NPs, Raman signals are 
readily detectable at a desirably low laser power and maximized when multiple NPs aggregate around the reporter molecules. In spite of this, extrinsic methods cannot obtain potentially important biochemical information directly from the protein in question. Intrinsic methods conversely rely on proteins' native spectral fingerprint as a means for detection. Although it is potentially more versatile, gathering intrinsic Raman data remains challenging when considering the extreme similarities among protein spectra as well as weak signal strength.

The Raman spectrum of a protein represents the biochemical fingerprint of the protein of interest. The identification of peaks in order to determine what biochemical structures give rise to certain peaks is extremely important for deconvolution of spectra generated from multiple proteins. Peak locations and their interpretations within the fingerprint region of biomolecules have been tabulated in great extent by Movasaghi et al. ${ }^{79}$ to assist researchers in this effort. Some of the following methods to be discussed have been demonstrated to achieve both intrinsic and extrinsic detection. As a result, instances of intrinsic detection are included in the aptamer, NP constructs, and magnetic field subcategories of the next section.

\subsubsection{Extrinsic Detection of Proteins}

\section{Using aptamers to enhance specificity to proteins}

Aptamers are single stranded oligonucleotides engineered to specifically bind to target sites on proteins or small molecules with high affinity and specificity. They are seen as a potentially more robust and stable alternative to antibodies as they do not exhibit significant cross-reactivity leading to non-specific linkage. Thrombin is of particular interest in past studies as the same aptamer 
can bind to two distinct locations on this protein; thus eliminating the need for an aptamer pair. Such protein specific aptamer pairs are scarce for the majority of proteins as their production is significantly difficult. ${ }^{80}$

Protein specific detection of $\alpha$-thrombin down to a detection limit of $0.5 \mathrm{nM}$ was reported by Wang et al. ${ }^{81}$ This was achieved by immobilizing thiolated thrombin binding aptamer (TBA) on an Au surface onto which thrombin binds. Subsequently, detection was achieved by attaching a second TBA that is thiolated to a AuNP modified with Raman reporters Rhodamine 6G (R6G). The reporters' signal was enhanced by addition of AgNPs and allowing the mixture to dry. In a similar assay-type scheme, Fabris et al. ${ }^{82}$ reported $100 \mathrm{pM}$ detection of thrombin using aptatags that consisted of AgNP dimers linked by the reporter molecule biphenyl-4,4'-dithiol (DBDT). Multiplexed detection of $100 \mathrm{pM}$ concentrations was later demonstrated by Fabris et al. ${ }^{83}$ via an expansion of the same technique using three unique SERS reporters for detecting thrombin, IgE, and platelet-derived growth factor-BB (PDGF-BB) using 1,4-benzenedithiol (BDT), 2,6-naphthalenedithiol (2,6-NDT), and DBDT, respectively. Recognition of IgE and PDGF-BB used aptamer pairs. Ligand exchange processes among aptamers due to non-specific adsorption was minimized by modifying NPs with PEG chains.

In an intrinsic detection scheme, Pagba et al. ${ }^{84}$ reported thrombin detection using a chair-type G-quadruplex structured TBA, which is advantageous due to its compactness (approximately $1.1 \mathrm{~nm}$ ) allowing the protein molecule to reside closer to the metal surface. This study reported the maximum allowable distance from the metal surface not to exceed $10 \mathrm{~nm}$ in order to obtain local SERS enhancement. The appearance of new peaks commonly characteristic of 
proteins $\left(1140,1540\right.$, and $\left.1635 \mathrm{~cm}^{-1}\right)$ was observed after the addition of thrombin, and was otherwise absent with the addition of BSA. Lastly, He et al. ${ }^{85}$ detected the intrinsic signal of ricin $\mathrm{B}$ chain with low detection limits of 10 $\mathrm{ng} / \mathrm{mL}$ in phosphate buffered saline (PBS), $50 \mathrm{ng} / \mathrm{mL}$ in orange juice, and 100 $\mathrm{ng} / \mathrm{mL}$ in milk using an aptamer linked to silver dendrites. Detection was accomplished within 40 minutes by a two-step procedure of ricin $\mathrm{B}$ chain binding to the aptamer followed by obtaining the Raman spectrum. High sensitivity of these techniques is ideal for bioreactor systems where proteins at minute concentrations need faster and cheaper detection than conventional methods.

\section{Using NP constructs to enhance Raman signals}

Another class of SERS aggregates, which are unlike conventional NP aggregates assembled by addition of a flocculent or immobilization, are composite organic-inorganic nanoparticles (COINs) that are linkable to a greater variety of Raman reporters compared to typical labeling methods as it does not require isothiocyanide and thiol groups, typically used for covalent linkage. This is achieved by a unique synthesis process described by Su et al. ${ }^{86}$ Briefly, Raman labels residing on the Ag surface facilitate aggregation by decreasing electrostatic repulsion between Ag particles. This study achieved multiplexed detection of interleukin-2 and interleukin-8 using COINs labeled with 8-azaadenine and $\mathrm{N}^{6}$-benzoyladenine, respectively. Raman reporters were also combined to produce unique sets of COIN signatures. Sun et al. ${ }^{87}$ expanded COINs to achieve tissue imaging as a Raman spectroscopic version of immunohistochemistry. In this study, cytokeratine-18 and prostate-specific antigen (PSA) immobilized on aldehyde glass slides were simultaneously detected and differentiated at various concentrations ( 1 to $10 \mathrm{ng} / \mathrm{mL}$ for PSA) 
using COINs labeled with the Raman active molecule Basic Fuchsin (BFU). Resultant peaks were relatively sharp and intense making it easy to distinguish even with a strong autofluorescence background. However, quantification remained limited due to inconsistent signal intensities among individual COINs originating from the synthesis process. Prostate-specific antigen (PSA) on prostate formalin-fixed paraffin embedded tissue samples was mapped with high sensitivity and specificity. COINs at extremely low concentrations (30 $\mathrm{pM}$ ) enabled rapid detection in as short as 0.1 seconds per pixel using a low excitation laser power $(0.3 \mathrm{~mW})$. In a later study, COINs was shown to possess comparable performance to Alex-ab conjugates on PSA detection by Lutz et al. ${ }^{88}$ Proteins indicative of T-cells residing on the surface of single cells were detected in human blood samples using COINs by Shachaf et al. ${ }^{89}$ with comparable performance to flow cytometry. Acridine orange and BFU were chosen as Raman labels. In comparison to current methods, only one staining step is required to introduce multiple optical labels thus reducing the number of tissue samples required for obtaining rich, multiplexed molecular information.

Silver NP dimers were synthesized via a process developed by Guarrotxena et al. ${ }^{90}$ and were used to achieve a detection limit of $100 \mathrm{fM}$ of immobilized human $\alpha$-thrombin in an assay-type setup. These dimers termed "anti-tags" were linked together with dithiolated DBDT-Raman reporter and further modified with antibody functionalized PEG as well as ordinary PEG for added stability in solution. Compared to the detection limit of ELISA (approx. 10 pM), this method provided approximately 100-fold improvement. This study demonstrates the potential of SERS assays to succeed immunoassays as it has comparable sensitivity and reproducibility without the need for prolonged procedures and expensive reagents. 
Han et al. ${ }^{91}$ reported the detection of proteins using a sandwich architecture platform consisting of three layers whereby the middle layer contained the target analytes. This method claimed to achieve greater reproducibility than other silver SERS methods. The base layer is a self-assembled layer of AuNPs on a cationic poly(diallyldimethylammonium chloride) (PDDA) coated glass surface. Target analytes are subsequently adsorbed onto the first layer and covered by a second, self-assembled layer of Au or AgNPs. Relevant to our discussion, avidin/biotin/atto610 complex was detected with greater reproducibility than that of silver aggregate methods. This method was shown to also intrinsically detect cytochrome c (cyt c). High reproducibility is imperative when precision is necessary in discerning multiple proteins for monitoring cell culture for engineering tissue.

\section{Using encoded nanoparticles to facilitate multiplexed detection}

An exciting approach that enables reproducible fabrication of microbeads labeled with a selected combination of SERS markers are the encoded nanoparticles with capabilities to potentially achieve multiplexed protein detection. An immunoassay type detection scheme of streptavidin and p53 was achieved by Jun et al. ${ }^{92}$ using polystyrene beads with surface-modified AgNPs and Raman labels. More importantly, it was shown that various molar ratios of Raman label chemicals can be used to make a wide variety of unique markers resolvable by the analysis of band intensity and position. More than 10,000 signatures are possible as reported by Kim et al. ${ }^{93}$ that used SERS-dots (AgNPs possessing attached Raman labels) to identify short peptide sequences bound to TentaGel beads with SERS-dots adsorbed onto the surface. Although entire protein molecules were not detected, there is still enormous potential in this 
method as we progress to more sophisticated computational deconvolution methods that will enable us to extrapolate valuable information from a massive number of possible signatures. For example, methods in biochemical component analysis ${ }^{94}$ could be used to quantify the contribution from each Raman reporter.

\section{Using magnetic fields to induce aggregation}

The surface enhancement effect is dramatically increased by shortening of distance as well as close aggregation of multiple nanoparticles to cause coupling of local electromagnetic fields ${ }^{41}$. The dramatic increase in signal intensity caused by multiple NPs residing in the immediate surroundings of the molecule is most desirable and has incited the development of aggregation methods. Magnetically induced aggregation of magnetic nanoparticles has been shown to cause no protein denaturation. ${ }^{95}$ In a later study by Jun et al. ${ }^{96}$, silver-embedded magnetic NPs (Ag-M-dots) that consisted of silica-coated magnetite NPs modified with thiol functional groups on the surface for AgNPs linkage were synthesized. The Raman signal from the reporters aminothiophenol (4-ATP) and benzenethiol (BT) displayed a 1000-fold increase after aggregation. This method also intrinsically detected adenine adsorbed onto the metal surface down to $1 \mathrm{pM}$ after magnetic aggregation. Although iron-based NPs have no magnetic memory, re-dispersion of the particles was facilitated by sonication. In a similar study by Chen et al. ${ }^{97}$, silver magnetic NPs (AgMPs) were prepared using AuNPs as nucleation sites for Ag shell formation and this enabled a low detection limit of $5 \mathrm{pg} / \mathrm{mL}$ for avidin. In this setup, avidin was bound to atto610-biotin molecule where atto610 served as the reporter molecule and was detected upon aggregation. The signal intensity of atto610 decreased as amounts of AgMPs increased, which 
demonstrated the dependence of high SERS signal strength on high concentration of protein per unit area. Furthermore, as the interaction time between atto610-biotin and avidin was increased to allow for binding, an increase in SERS signal was observed for up to 60 minutes. Magnetic silver NPs thus hold great potential for protein detection as well as intracellular monitoring in TERM.

\subsubsection{Intrinsic Detection of Proteins}

Assay-type schemes

Assay methods presented here exploit the recognition and binding of antibodies to corresponding proteins as well as binding between compatible biomolecules, such as the binding reaction of avidin and biotin, to identify target bioanalytes using RS instead of enzymatically generated signals and radioactive labels used in current methods. Reliably discerning a single protein species within a background of foreign proteins using spontaneous RS remains unachieved. Such a breakthrough would revolutionize TERM diagnostic methods. SERS detection may hold the solution as demonstrated by He et al. ${ }^{98}$ to detect a single protein type within a complex protein matrix. Silver dendrites modified with protein $\mathrm{G}$ and further bound to an antibody, anti-OVA, were used to detect ovalbumin (OVA) in milk at $0.1 \mu \mathrm{g} / \mathrm{mL}$ and in PBS at $5 \mu \mathrm{g} / \mathrm{mL}$ in about 30 minutes. Given that signal enhancement due to the SERS effect applies to all the aforementioned molecules, PCA was employed to differentiate between dendrites bound and not bound to OVA. Comb et al. ${ }^{99}$ fabricated a novel SERS surface micropatterned with AgNPs into long, narrow strips. Positively charged biomolecules selectively bound to the negatively charged SERS active sites (silver) while the surface nanocoating prevented adsorption in the remaining area. Among the numerous advantages of this highly ordered 
arrangement, it dramatically reduced interfering background from the substrate and nonspecifically adsorbed species making it ideal for minute concentration detection. In this study, the binding reaction of IgG to Protein A was exploited after it firstly adsorbed onto the patterned regions in order to gather their SERS spectra. Using a 30 second exposure time, detection limits of $5 \mathrm{nM}$ and $500 \mathrm{fM}$ for Protein A and IgG, respectively, were reported. These methods have the potential to supersede current assay techniques by negating the need for expensive reagents and lengthy procedures.

\section{Optofluidics to enhance aggregation}

Wang et al. ${ }^{100}$ used the entrapping mechanism of micro- to nano-fluidic channels to form a cluster of AuNP and analyte molecules at the entrance of a nanochannel. The device, described as "optofluidic", was fabricated on a borosilicate wafer, and successfully detected adenine with a lower limit of 10 pM. A subsequent study conducted by Chou et al. ${ }^{101}$ also demonstrated the use of this device to detect $\beta$-amyloid peptide at $11.5 \mathrm{pM}$ as a biomarker for potential premortem diagnosis of Alzheimer's disease. In a more recent study by Wang et al. $^{43}$, an optofluidic device on silicon substrate was fabricated to detect structural changes of albumin and insulin induced by elevating temperature. As temperature was increased from room temperature to $100^{\circ} \mathrm{C}$, protein denaturation was successfully observed as a few band intensities decreased, particularly at $1277 \mathrm{~cm}^{-1}$ which corresponds to the $\alpha$-helical secondary structure. Low protein concentrations in the range of $0.9 \mathrm{ng} / \mathrm{L}$ of albumin and insulin were detectable at an excitation power of $70 \mathrm{~mW}$ and an exposure time of 60 seconds. This group reported that 16 of these devices can be inexpensively made on one 4-inch wafer potentially allowing for parallel analysis and high throughput. Moreover, only microliter amounts of volume is 
required for operation. Such fluidic channels could be integrated into a microfluidic system for tissue engineering ${ }^{102}$ to enable real-time monitoring. Sensitivity to such minute concentrations as well as the low sample volume makes this method more than ideal to monitor tissue bioreactors cheaply and non-invasively.

\section{Detection of protein in solution using SERS}

Selective identification of target proteins in solution is most desirable for detecting soluble proteins present in tissue bioreactors. It not only eases sample preparation, but solution measurements are also highly reproducible. Binary mixtures containing cyt $\mathrm{c}$ and cytochrome $\mathrm{b}_{5}\left(\mathrm{cyt}_{\mathrm{t}} \mathrm{b}_{5}\right)$ were selectively enhanced by exploiting their opposite surface charges ${ }^{103}$ using surface-enhanced resonance Raman scattering. Two batches of AgNPs were coated with $\mathrm{SiO}_{2}$ and chitosan to prevent heme-protein denaturation. When protein molecules came into contact with NPs, spectra of the native structure was obtained. Furthermore, as cyt c was dominated by positively charged amino acid residues, electrostatic attraction to negatively charged AgNPs became highly favorable. This led to strong enhancement of only cyt $\mathrm{c}$ in the presence of $\mathrm{SiO}_{2} \mathrm{AgNPs}$. Conversely, negatively charged cyt $b_{5}$ were not enhanced due to electrostatic repulsion. However, exclusive enhancement of cyt $b_{5}$ was observed with the addition of chitosan-coated AgNPs as chitosan exhibits high affinity for anionic biomolecules. A highly reproducible and flexible method developed by Yang et al. ${ }^{104}$ achieved ultra-low concentration detection of lysozyme and cyt c using a tip-coated multimode fiber (TCMMF). The negatively charged silica fiber tip was coated with cationic cetyltrimethylammonium bromide (CTAB) modified AgNP. Subsequent dipping of the fiber into a bulk solution containing anionic citrate-reduced AgNPs and the attached target protein resulted in electrostatic 
attraction that reduced the distance between the two NP types. Strong SERS spectra, even when using a portable Raman system, were detectable down to $0.2 \mu \mathrm{g} / \mathrm{mL}$ using this method. Acidified sulfate was found by Han et al. ${ }^{105}$ to be an excellent aggregation agent for SERS detection of proteins. As low as 50 $\mathrm{ng} / \mathrm{mL}$ of catalase and $5 \mu \mathrm{g} / \mathrm{mL}$ of lysozyme was detected by this simple protocol that mixes AgNPs and proteins with $\mathrm{Na}_{2} \mathrm{SO}_{4}$. It was hypothesized that weak binding strength of sulfate anions to AgNPs made it easier for proteins to access the AgNP surface. The expansion of these methods to have greater multiplexing capability would offer a swifter alternative to current assay-type schemes.

\section{Aggregation on dried surface}

Molecular packing caused by drying of a metal NP and biomolecule colloid solution can radically improve SERS performance. Dried sample solutions of binary mixtures of chromophore and non-chromophore containing proteins were subjected to SERS by Zhou et al. ${ }^{106}$ In this quantitative study, increasing the concentration of one protein in homogenous chromophore ( $\mathrm{Mb}$ and cyt $\mathrm{C}$ ) and non-chromophore (apoMb and lysozyme) mixtures corresponded to greater band intensities. However, in heterogeneous binary mixtures, sequential addition of the chromophore containing protein resulted in a bell-shaped variation in Raman intensities corresponding to the protein, which is an important phenomenon to note during multiplexed SERS studies of such proteins. The primary drawback of drying droplets is uneven distribution of analytes and NPs where over-jamming of molecules typically occurs at the droplet's edge. Keskin et al. ${ }^{107}$ attempted to dry AgNP mixed with protein colloid by suspending the droplet from a hydrophobic surface. This resulted in concentration of proteins at the center of the droplet after drying regardless of 
protein charge, shape, and size. As low as $0.05 \mu \mathrm{g} / \mathrm{mL}$ of human serum albumin (HSA), transferrin, myoglobin, and avidin were detected with high spectral quality and reproducibility using this simple method. Drying dynamics were also modified by Culha et al. ${ }^{108}$ by dipping a plastic tip into the center of a sample during the drying process that altered packing behavior at the edges. Anionic BSA exhibited one order of magnitude improvement in the detection limit compared to the ordinary drying protocol. Conversely, cationic cyt c exhibited no enhancement in the detection limit. This technique offers a straightforward approach to promptly detect minute quantities when protein specificity is not required. 


\section{Convective assembly}

Convective assembly is a controlled process whereby structured patterns consisting of metal NPs and protein molecules are developed from thin evaporating films of colloidal mixtures. This technique exploits the unique distribution behavior of various proteins and successfully differentiates them in a reproducible and label-free manner. ${ }^{109}$ Kahraman et al. ${ }^{110}$ reported that pattern formation depends on protein charge and size when considering the relative charge polarities between proteins and NPs. Negatively charged proteins were observed to form small aggregates due to weak interaction with the anionic AgNPs while positively charged proteins formed larger aggregates owing to electrostatic attraction to the AgNPs. Concentrations as low as $0.5 \mu \mathrm{g} / \mathrm{mL}$ were detectable for BSA, catalase, pepsin, cyt c, avidin, and lysozyme when using relatively low laser power (about $3 \mathrm{~mW}$ ) and short exposure times (about $10 \mathrm{~s}$ ) to produce SERS spectra with excellent quality and reproducibility. It was later revealed by Keskin et al. ${ }^{111}$ that differences between sedimentation behaviors among proteins result in unique distribution patterns when binary and ternary mixtures are assembled. Briefly, in the situation involving negatively charged proteins, multiple spectra taken from a series of regions along the length of the convective assembly revealed that larger proteins tend to sediment before smaller ones. In the alternate case of positively charged proteins, the opposite was observed where higher intensity bands indicative of smaller proteins were detected in the initial regions. However, high molarity of the protein used (cyt c) relative to its counterpart (avidin) may have facilitated electrostatic attraction with negatively charged AgNP. A recent investigation by Kahraman et al. ${ }^{112}$ examined the effect of surface hydrophobicity on aggregation behavior and SERS spectral quality. A distinct difference in aggregation behavior and SERS enhancement were observed between assemblies on hydrophilic and 
hydrophobic surfaces where the use of a hydrophobic surface resulted in a thin film composed of cationic protein and NPs compared to the formation of aggregates with the use of a hydrophilic surface. The same aggregation behavior was observed, only less pronounced, for anionic proteins owing to increased interaction between protein and NPs caused when sandwiched between two hydrophobic environments. In terms of SERS enhancement, using hydrophobic surfaces with $16 \times 10^{11} \mathrm{NPs} / \mathrm{mL}$ provided high reproducibility and sensitivity. A lower limit concentration of $0.5 \mu \mathrm{g} / \mathrm{mL}$ for BSA, hemoglobin, thrombin, avidin, cyt c, and lysozyme was reported. Overall, protein separation was achieved label-free, and this method may contribute to more robust protein differentiation methods in the future. 
Western SERS

Western SERS is a combined approach that utilizes techniques in western blotting for the separation of proteins followed by detection using SERS technique. In this method reported by Han et al. ${ }^{113}$, multiple proteins were identified by Raman signal detection on one nitro cellulose (NC) membrane while avoiding the need for external labels, extraction, and/or mass spectrometry that are required in typical Western blotting/proteomics procedures. A protein mixture of myoglobin $(\mathrm{Mb})$ and BSA was separated on native polyacrylamide gel electrophoresis (PAGE) and transferred onto a NC membrane via electroblotting as per traditional western blotting procedure. Subsequently, characteristic Raman spectra of the two protein bands were obtained using confocal RS. The SERS signal was obtained after soaking the NC membrane in silver colloid solution to allow adsorption of AgNPs, and this resulted in a low detection limit of $4 \mathrm{ng} / \mathrm{band}$ of $\mathrm{Mb}$ and $8 \mathrm{ng} / \mathrm{band}$ of BSA. This method could be easily implemented into current Western blotting procedures to shorten processing time, improve sensitivity, and reduce the requirement of expensive reagents. 
Table 2.1 Summary of applications, techniques, references, and applicable detection limits of proteins using RS

\begin{tabular}{|c|c|c|c|c|}
\hline \multicolumn{2}{|l|}{ Application } & $\begin{array}{l}\text { Technique } \\
\text { Aptamers }\end{array}$ & \multirow{2}{*}{$\begin{array}{l}\text { Reference } \\
\text { Wang et al. (81) }\end{array}$} & \multirow{2}{*}{$\begin{array}{l}\text { Lowest Detected } \\
\text { Protein } \\
\text { Concentration } \\
0.5 \mathrm{nM} \alpha \text {-thrombin }\end{array}$} \\
\hline $\begin{array}{l}\text { Extracellular } \\
\text { Protein } \\
\text { Detection }\end{array}$ & $\begin{array}{l}\text { Extrinsic } \\
\text { Methods }\end{array}$ & & & \\
\hline \multirow{19}{*}{ Detection } & & & Fabris et al. (83) & $\begin{array}{l}100 \text { pM thrombin, } \\
\text { IgG, PDGF-BB }\end{array}$ \\
\hline & & & $\begin{array}{l}\text { Pagba et al. (84) } \\
\text { (intrinsic) }\end{array}$ & $\begin{array}{l}\text { Thrombin. No } \\
\text { lower limit } \\
\text { reported }\end{array}$ \\
\hline & & & He et al. (85) (intrinsic) & $\begin{array}{l}10 \mathrm{ng} / \mathrm{mL} \text { ricin B } \\
\text { Chain }\end{array}$ \\
\hline & & $\begin{array}{l}\text { Composite Organic- } \\
\text { Inorganic NPs (COINs) }\end{array}$ & $\begin{array}{l}\text { Sun et al. (86) } \\
\text { Sun et al. (87) }\end{array}$ & $\begin{array}{l}\text { Interluekin-2, } \\
\text { interleukin-8. } \\
\text { No lower limit } \\
\text { reported }\end{array}$ \\
\hline & & & & $1 \mathrm{ng} / \mathrm{mL}$ PSA \\
\hline & & Nanoparticle Constructs & $\begin{array}{l}\text { Guarrotxena et al. (90) } \\
\text { Han et al. (91) }\end{array}$ & $\begin{array}{l}100 \mathrm{fM} \text { human } \alpha- \\
\text { thrombin }\end{array}$ \\
\hline & & & & $\begin{array}{l}\text { Avidin, cytochrome } \\
\text { c (intrinsic). No } \\
\text { lower limit } \\
\text { reported. }\end{array}$ \\
\hline & & Encoded Nanoparticles & Jun et al. (92) & $\begin{array}{l}\text { No lower limit } \\
\text { reported }\end{array}$ \\
\hline & & $\begin{array}{l}\text { Magnetically Induced } \\
\text { Aggregation }\end{array}$ & $\begin{array}{l}\text { Jun et al. (96) } \\
\text { (intrinsic) }\end{array}$ & $\begin{array}{l}1 \mathrm{pM} \text { adenine } \\
\text { (nucleobase) }\end{array}$ \\
\hline & & & Chen et al. (97) & $5 \mathrm{pg} / \mathrm{mL}$ avidin \\
\hline & $\begin{array}{l}\text { Intrinsic } \\
\text { Methods }\end{array}$ & Assay & $\begin{array}{l}\text { He et al. (98) } \\
\text { Comb et al. (99) }\end{array}$ & $\begin{array}{l}0.1 \mu \mathrm{g} / \mathrm{mL} \text { OVA } \\
5 \mathrm{nM} \text { protein A, } 500 \\
\mathrm{fM} \text { IgG }\end{array}$ \\
\hline & & Optofluidics & Wang et al. (100) & $10 \mathrm{pM}$ \\
\hline & & & Chou et al. (101) & 11.5 pM $\beta$-amyloid \\
\hline & & & Wang et al. (43) & $\begin{array}{l}0.9 \mathrm{ng} / \mathrm{L} \text { albumin, } \\
\text { insulin }\end{array}$ \\
\hline & & SERS in Protein Solutions & Sivanesan et al. (103) & $\begin{array}{l}\text { Cyt } c \text {, cyt } b_{5} \text {. No } \\
\text { lower limit } \\
\text { reported }\end{array}$ \\
\hline & & & $\begin{array}{l}\text { Yang et al. (104) } \\
\text { Han et al. (105) }\end{array}$ & $\begin{array}{ll}0.2 \mu \mathrm{g} / \mathrm{mL} \\
\text { lysozyme, cyt c }\end{array}$ \\
\hline & & & & $\begin{array}{l}50 \mathrm{ng} / \mathrm{mL} \text { catalase, } \\
5 \mu \mathrm{g} / \mathrm{mL} \text { lysozyme. } \\
\text { No lower limit } \\
\text { reported for } \\
\text { ribonuclease B, } \\
\text { avidin, and } \\
\text { hemoglobin. }\end{array}$ \\
\hline & & $\begin{array}{l}\text { Aggregation on Dried } \\
\text { Surface }\end{array}$ & $\begin{array}{l}\text { u et al. (106) } \\
\text { kin et al. (107) }\end{array}$ & $\begin{array}{l}\text { No lower limit } \\
\text { reported }\end{array}$ \\
\hline & & & & $0.05 \mu \mathrm{g} / \mathrm{mL}$ human \\
\hline
\end{tabular}




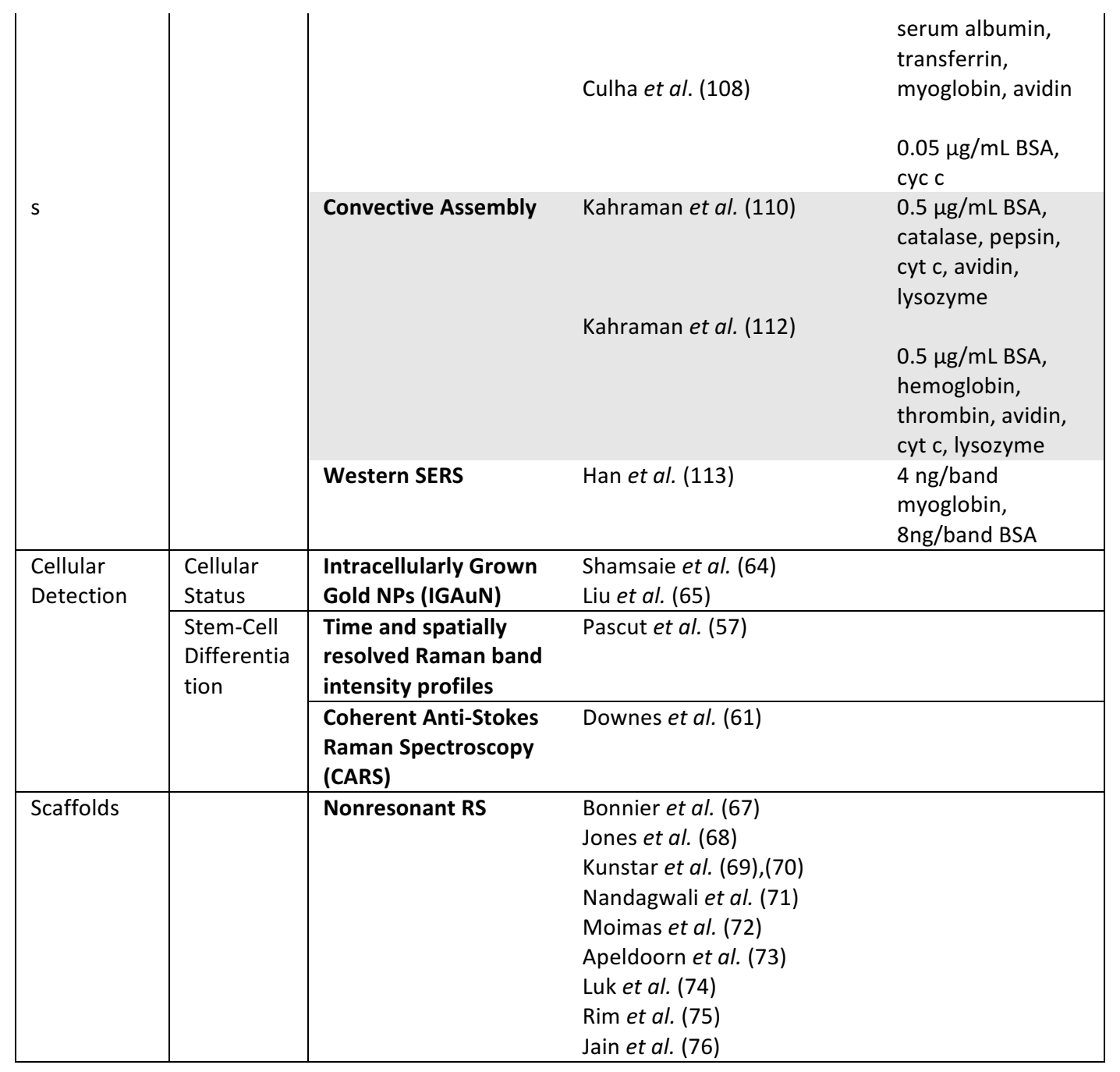


Table 2.1 summarizes the applications and techniques that have been discussed in this chapter involving the use of Raman spectroscopy and includes information on the detection limits of proteins, where applicable, that are relevant or potentially applicable to tissue engineering.

With the current commercial availability of Raman probes capable of liquid immersion comes the ability for in situ detection of media analytes in tissue bioreactors. Raman spectroscopy was recently used to monitor multiple media components such as glutamine, glutamate, glucose, lactate, ammonium, and viable/total cell density simultaneously by partial least squares regression in Chinese hamster ovary cell culture bioreactors. ${ }^{114,115}$ Although this technique has potential to eliminate high costs associated with usage and maintenance of current-day sensors/biosensors, the high fluorescence of biomolecules that overlap spectral bands remains a major challenge. A near infrared excitation laser and using shifted-subtracted RS can largely minimize this problem ${ }^{116}$, but further research is expected to develop and refine this technique for commonplace use.

\subsection{Conclusion and Future Prospects}

This chapter has provided an overview of Raman spectroscopy (RS) based techniques and captured the capability of RS to noninvasively extract biochemical information from cells, scaffolds, and proteins through a variety of methods and approaches. In essence, it has truly demonstrated that the potential applications for TERM are widespread.

The overwhelming evidence covered in this chapter further supports and illustrates the inevitable development of Raman spectroscopy in future TERM 
diagnostics. No other similar modality possesses the noninvasive and nondestructive versatility to monitor multiple biochemical analytes in situ. In order to realize a landmark paradigm to grow tissue replacements optimally and reproducibility for clinical use, the availability of such capabilities to existing culture platforms is unquestionably necessary. As SERS methodologies have largely dominated protein detection, we anticipate continued progress to achieve greater quantitative accuracy, reproducibility, and specificity with the addition of more facile and multiplexed techniques. This trend will lead to RS based platforms that may eventually outperform traditional immunohistochemical techniques not only with respect to cost effectiveness and convenience but also technical competency. Moreover, the use of Raman spectroscopy in TERM will expand as the evolving complexity of tissue scaffolds continues to progress and require non-perturbing methods for cell interrogation. Further development of Raman techniques to monitor biological structures on a tissue, cellular, and molecular level will result in a comprehensive methodology to revolutionize tissue engineering and regenerative medicine. 


\section{Chapter 3 Polarized Raman Spectroscopy for Enhanced Quantification of Protein Concentrations in an Aqueous Mixture}

Significant portions of this chapter have been taken directly from a previously published paper entitled "Polarized Raman Spectroscopy for Enhanced Quantification of Protein Concentrations in an Aqueous Mixture.”, in which the first author is the author of this dissertation. The remaining two authors, listed here in the appropriate order, Dr. Sierin Lim and Dr. Quan Liu, are hereby acknowledged for their valued contribution.

\subsection{Introduction}

Protein quantification has been ubiquitous and essential throughout all fields involving biology such as gene expression studies ${ }^{117,118}$, disease diagnosis ${ }^{119-}$ 121, and tissue engineering. ${ }^{122,123}$ Conventional methods for quantifying proteins, as identified in the previous chapter, are ill-suited for fast and selective monitoring of protein concentration in mixtures.

Raman spectroscopy (RS) has shown great potential to complement conventional methods for protein quantification. Fast and selective quantification of proteins within mixtures then becomes possible without the burden of slow and tedious protein separation procedures.

While multiplexed detection of proteins using extrinsic Raman reporters has achieved great success ${ }^{87,124-127}$, it is frequently necessary to perform this task using intrinsic Raman measurements such as in monitoring tissue bioreactors where non-destructive measurements are much more desirable. There are several challenges to overcome in order to apply RS to the quantification of 
proteins in this situation such as intrinsically weak Raman signals and high fluorescence background that cannot be easily addressed by traditional RS alone. Furthermore, another major challenge is high similarity among protein spectra, making it difficult to discriminate between protein species and accurately measure the concentration of each. For this reason, the quantitative accuracy and robustness of spectral deconvolution methods such as principal component analysis (PCA) and biochemical component analysis (BCA) are limited when only traditional RS is applied. Multiplexed detection of proteins has been extensively explored ${ }^{128}$ and highly sensitive detection of solvated proteins has been reported. ${ }^{45,129,130}$

Polarized Raman spectroscopy (PRS) offers a unique solution to enhance the accuracy of analyte quantification without significantly increasing the cost of the system. Data from conventional RS for quantification is limited to a single spectrum that may only contain a constrained amount of data for quantification. In contrast, PRS avails multiple spectra ${ }^{131}$ by measuring Raman spectra with different polarizations from which additional data with complementary information can be acquired to improve selective quantitative accuracy in aqueous protein mixtures.

PRS is a powerful technique used predominantly for determining the orientation of molecules in highly ordered structures. ${ }^{132}$ This technique provides a means to exploit the polarization properties of scattered Raman radiation to selectively view spectral information created by symmetric and asymmetric molecular vibrations. ${ }^{1}$ 
Molecules exposed to a plane polarized laser source sets into motion symmetric and asymmetric molecular vibrations that release Raman photons polarized in a variety of angles relative to that of the excitation laser. In the symmetric case, scattered Raman light is polarized in the same plane as the incident laser beam whereas photons in the asymmetric case may not be. This property remains true even for molecules randomly oriented in solution ${ }^{133}$, which may offer additional information for helping differentiate proteins with highly similar Raman spectra in traditional RS. This additional information can be utilized in the nondestructive detection and selective quantification of multiple proteins in a mixture.

In this work, we demonstrated for the first time that PRS can significantly improve protein quantification compared to traditional RS. A new method that takes advantage of the additional spectra offered by PRS was developed to select highly sensitive peaks for accurate protein concentration estimation. This method was evaluated in mixed lysozyme/ $\alpha$-amylase solutions at various concentrations to investigate the possibility of enhanced peak sensitivity as a function of polarization to improve quantification accuracy.

A PRS optical setup was constructed, which featured an analyzer for selectively viewing scattered light either parallel or perpendicular to the polarization of the excitation beam. Spectra of mixed solutions and basic component spectra of the proteins alone were collected in both parallel and perpendicular configurations in addition to the conventional Raman configuration where the analyzer was removed. Peaks were evaluated in both polarizations and selected based on which polarization yielded greater sensitivity to create a unique combination of peaks for optimizing concentration estimation. The linear sum 
of each protein signal was assumed to constitute the total Raman signal emitted by the mixture. Application of our peak-selection criteria yielded significant improvement in quantification accuracy relative to a traditional Raman detection scheme without selective polarization capability. 


\subsection{Materials and methods}

\section{Solutions}

Lysozyme and $\alpha$-amylase were purchased as lyophilized powder (SigmaAldrich, Missouri, USA). These two proteins were selected because of their cost effectiveness without losing the generality of representing other more expensive proteins. All solutions were made in ultrapure MilliQ water. A dilution matrix of binary mixtures is given in Table 3.1. Concentrations used for basic component spectra ranged from $10 \mathrm{~g} \mathrm{~L}^{-1}$ to $50 \mathrm{~g} \mathrm{~L}^{-1}$ with an increment of $10 \mathrm{~g} \mathrm{~L}^{-1}$ for both proteins.

Table 3.1 Dilution matrix of binary protein mixtures of lysozyme and $\alpha$-amylase. In each cell, the first number indicates the concentration of $\alpha$-amylase and the second number indicates the concentration of lysozyme.

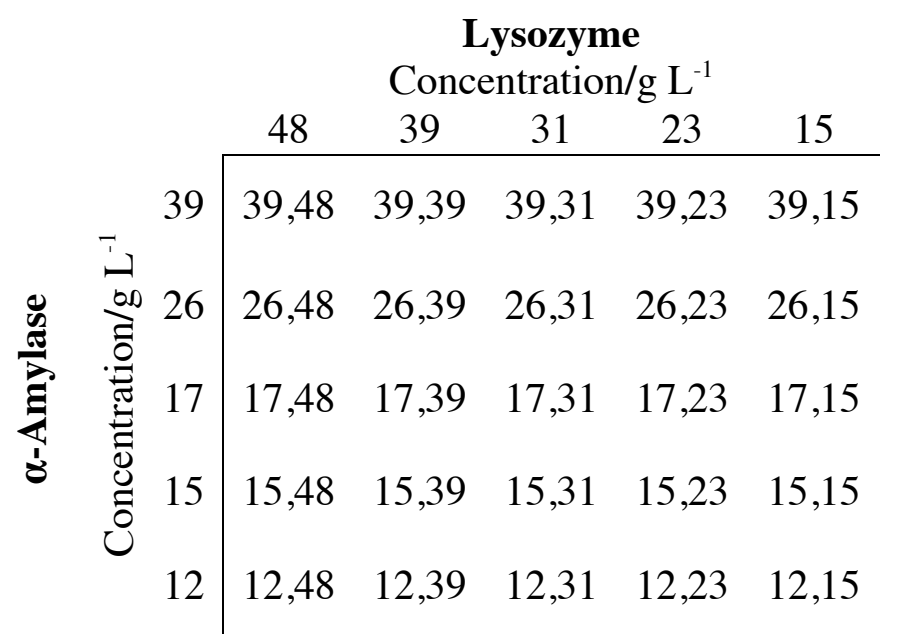

\section{Polarized Raman Spectra Acquisition}

A special optical setup for the acquisition of polarized Raman spectra was constructed as depicted in Figure 3.1. A right-angle scattering geometry was selected to avoid the use of a dichroic mirror that would vary in transmission and reflectivity with respect to the polarization of incoming and transmitted light. The scattering plane is defined here by the axis of incident propagation and the axis of scattering propagation. Therefore, in Figure 3.1, the scattering 
plane is the plane of the page, which will now be referred to as 'within-page'. Conversely, the plane perpendicular to the page will be referred to as 'intopage'. Raman spectra obtained with linear polarization parallel and perpendicular to that of the excitation light were generated using a rotatable half-wave plate placed in the excitation beam path and a fixed analyzer oriented such that its transmission axis was fixed perpendicularly to the scattering plane (into-page). ${ }^{134}$ The incident polarization was oriented into-page for making parallel measurements and later rotated to within-page for perpendicular measurements.

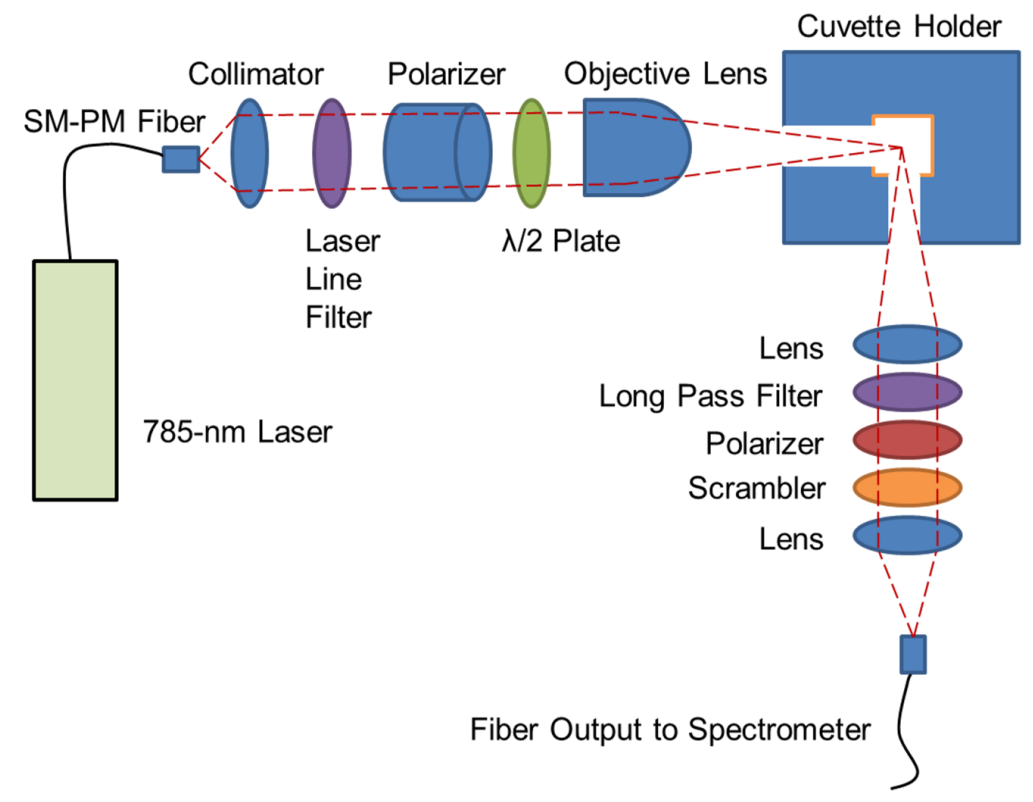

Figure 3.1 Polarized Raman Spectroscopy (PRS) optical setup diagram. "SM-PM Fiber" refers to a single-mode polarization maintaining fiber.

To verify the half-wave plate's parallel and perpendicular positions, the depolarization ratio, denoted by $\rho$, of chloroform was measured and compared to published values ${ }^{135}$ given in Table 3.2. Prior to this, the spectrometer was calibrated for wavelength using a factory provided neon lamp (Princeton Instruments, USB-Hg-NeAr, New Jersey, USA) and accompanying software. The $\rho$ value is defined in Equation 1 as the ratio of intensity in perpendicular 
$\left(I_{\perp}\right)$ to parallel $\left(I_{\|}\right)$polarizations at any selected wavenumber. ${ }^{1}$ Comparison in Table 3.2 showed excellent agreement with published values, and validated our optical setup for polarized Raman measurements. As with any neat organic solvent, Raman signals were markedly intense and required an exposure time of two seconds, which removed the need for cosmic ray removal and all other automatic data preprocessing options. Also, chloroform, being clear and colorless, generated a negligible amount of fluorescence that obviated the need for background subtraction.

$$
\rho \equiv \frac{I_{\perp}}{I_{\|}}
$$

Table 3.2 Comparison of depolarization ratios of selected bands of chloroform between values measured in this study and those in literature ${ }^{135}$

\begin{tabular}{ccc} 
Wavenumber $\mathrm{cm}^{-1}$ & $\rho$ (Measured) & $\rho$ (Literature Values) $^{135}$ \\
\hline 367 & $0.10 \pm 9 \times 10^{-4}$ & 0.09 \\
667 & $0.01 \pm 4 \times 10^{-5}$ & 0.01 \\
761 & $0.74 \pm 5 \times 10^{-3}$ & 0.72 \\
1217 & $0.74 \pm 1 \times 10^{-2}$ & 0.71 \\
3018 & $0.11 \pm 1 \times 10^{-2}$ & 0.10
\end{tabular}

Samples were excited by a diode laser (Toptica XTRA II) operating at $785 \mathrm{~nm}$ and coupled to a single-mode polarization maintaining fiber. Approximately $150 \mathrm{~mW}$ reached each sample. Scattered Raman light was firstly collimated by an achromatic lens then passed through a $785 \mathrm{~nm}$ long-pass filter, a polarizer, a quartz-wedge depolarizer (denoted as 'scrambler'), and finally focused onto a multimode fiber with a $200 \mu \mathrm{m}$ core size. The output end of the collection fiber was connected to a spectrometer (Acton LS 785, Princeton Instruments, New Jersey, USA) controlled by LightField software and coupled to super-cooled CCD (400BR_eXcelon, Princeton Instruments, New Jersey, USA) optimized 
for NIR detection. Automatic cosmic ray removal was activated. All PRS spectra were an average of three sequential exposures with an integration time of 22 seconds each.

\section{Traditional Raman Spectra Acquisition}

Ordinary Raman spectra were collected with the analyzer removed and the incident laser oriented such that its electric vector was perpendicular to the scattering axis to maximize the strength of detected Raman light. ${ }^{131}$ The integration time was 12 seconds in this configuration and three accumulations were averaged to generate an averaged spectrum. This setup mimicked those in most commercial Raman setups such as the Renishaw microRaman system (Gloucestershire, UK).

\section{Spectral Processing}

Background removal was performed using a wavelet-transform based method made freely available by Zhang et al. ${ }^{136}$ The relationship between peak intensity and concentration was analyzed for each peak to determine if any lacked a linear relationship that needed omission for concentration estimation (data not shown). The integrated areas under each peak covered a bandwidth of about $7 \mathrm{~cm}^{-1}$ that consisted of three sequential data points separated by an increment of about $3.5 \mathrm{~cm}^{-1}$. The areas were used for all spectral analyses and concentration estimations. In Figure 3.2 and Figure 3.3, a sample of raw spectra, the portion removed by background removal, and the residual are presented. The spectra of both amylase and lysozyme at concentrations of $50 \mathrm{~g}$ $\mathrm{L}^{-1}$ and $10 \mathrm{~g} \mathrm{~L}^{-1}$, respectively, taken in the parallel and perpendicular detection geometries are presented to provide a sense of how much the background removal process affected peak height. 

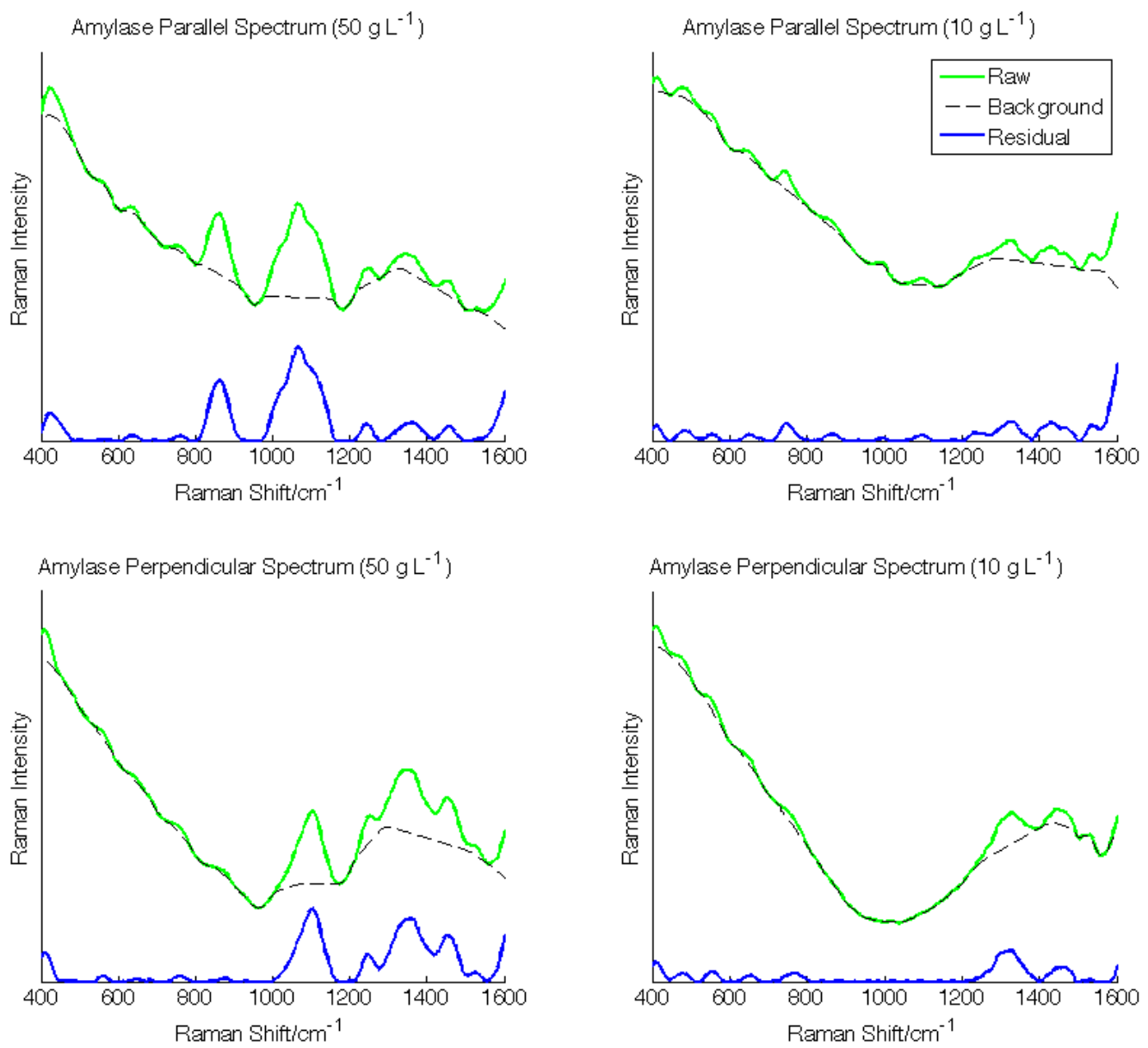

Figure 3.2 Spectra of amylase where raw spectra are shown in green, the calculated background in dashed black, and the residual in blue. The left column corresponds to parallel (top) and perpendicular (bottom) spectra at $50 \mathrm{~g} \mathrm{~L}^{-1}$ concentration. The right column corresponds to parallel (top) and perpendicular (bottom) spectra at $10 \mathrm{~g} \mathrm{~L}^{-1}$ concentration. 

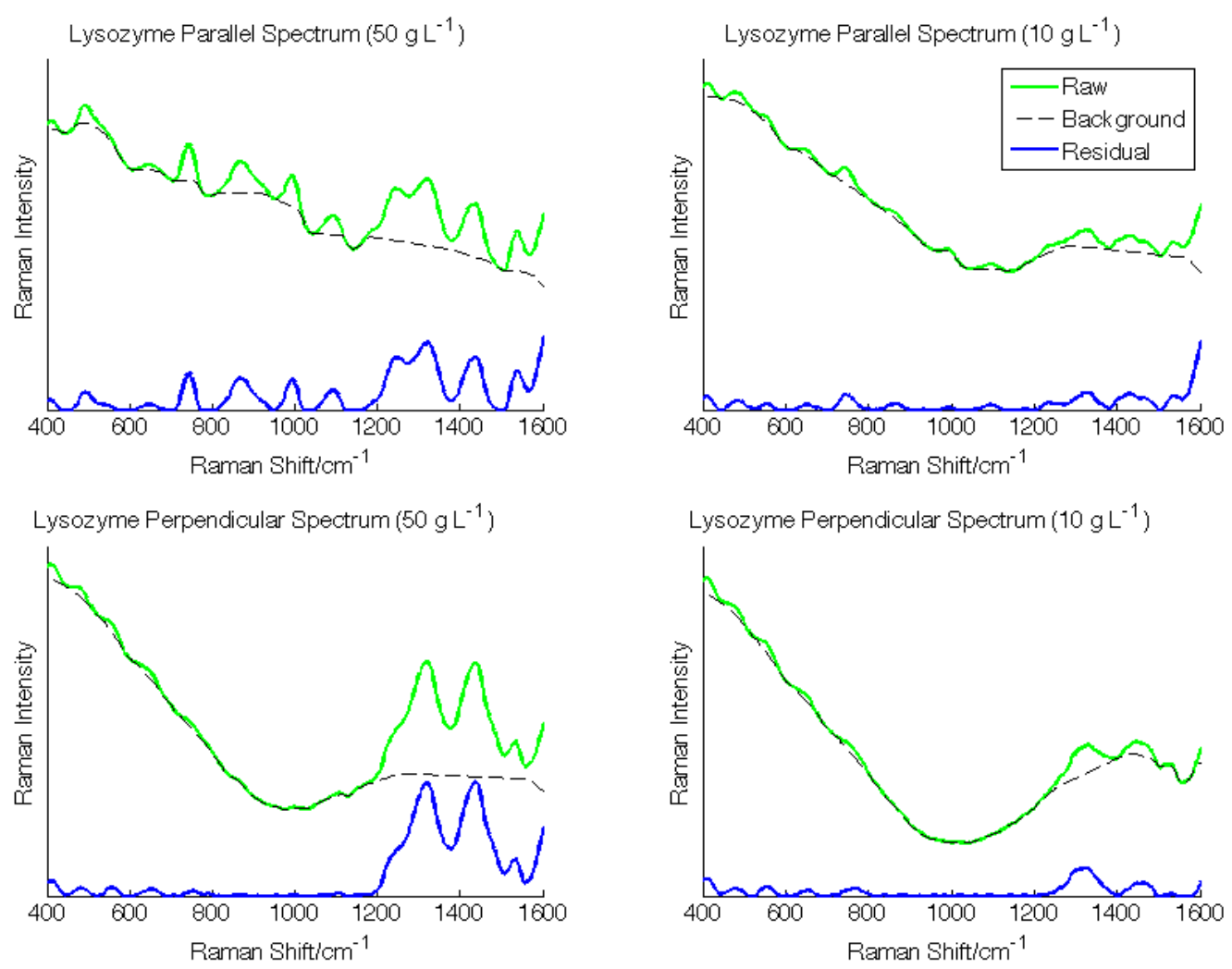

Figure 3.3 Spectra of lysozyme where raw spectra are shown in green, the calculated background in dashed black, and the residual in blue. The left column corresponds to parallel (top) and perpendicular (bottom) spectra at $50 \mathrm{~g} \mathrm{~L}^{-1}$ concentration. The right column corresponds to parallel (top) and perpendicular (bottom) spectra at $10 \mathrm{~g} \mathrm{~L}^{-1}$ concentration.

\section{Peak Selection Criteria and Non-Linear Least Squares Regression}

Concentration estimations from the traditional RS setup, or unpolarized case, used all selected peaks since there was only one spectrum available. However, this was not the case in polarized Raman spectra since there were two polarizations available. Out of the selected peaks for each protein, their sensitivity to change in response to its corresponding protein concentration variation was compared between parallel and perpendicular polarizations. Here the sensitivity of a Raman peak for a given protein was defined as the ratio of the change in the peak intensity to the variation in the protein's concentration, in which Raman spectra were measured from the solution of this particular protein alone. The set of slopes relating band area to protein concentration, $\boldsymbol{A}_{n}$, were normalized for comparison such that $\boldsymbol{A}_{n}$ for each protein and polarization 
was divided by the maximum of $\boldsymbol{A}_{n}$. The normalized values of $\boldsymbol{A}_{n}$ for all polarizations were compared. For each peak, the polarization that yielded the greatest sensitivity among parallel and perpendicular was selected and used to create a unique combinatorial set consisting of peaks from both polarizations.

The Raman signals emitted by each protein were assumed to add linearly thus making it possible to calculate their concentrations using a calibration curve made from their basic component spectra. Using data from multiple peaks and both polarizations, an over-determined system given by Equation 2, where $\boldsymbol{I}_{m}$ is the linear sum of signals from each protein, $C_{n}$ is the concentration of every nth species, $\boldsymbol{A}_{n}$ is a vector containing the slope of peak intensity versus concentration at each selected wavenumber denoted by $\lambda$ with the selected polarization, and $\boldsymbol{B}_{n}$ contains the corresponding y-intercepts, was solved. The subscript of the wavelength variable, $\lambda$, indicates that each wavenumber can correspond to either the parallel $(\|)$ or perpendicular $(\perp)$ polarization in accordance with the chosen combinatorial set of peaks. A non-linear least squares regression (NLLSR) algorithm, lsqcurvefit, provided in MatLab was used. The initial starting point value was $30 \mathrm{~g} \mathrm{~L}^{-1}$ with no other options selected. In the unpolarized case, Equation 2 reduces to Equation 3 where information is limited to just one spectrum. The concentration of each protein was estimated for every dilution shown in Table 3.1. The ultimate concentration values reported for $\alpha$-amylase were the average of all dilutions in each row in Table 3.1. Likewise, for lysozyme, the average was calculated for all dilutions in each column. These values were used to calculate the root mean squared error (RMSE) between estimated and actual concentration values.

$$
\boldsymbol{I}_{m}\left(\lambda_{\|} \text {or } \lambda_{\perp}\right)=\sum_{n=1}^{i}\left[C_{n} \boldsymbol{A}_{n}\left(\lambda_{\|} \text {or } \lambda_{\perp}\right)+\boldsymbol{B}_{n}\left(\lambda_{\|} \text {or } \lambda_{\perp}\right)\right]
$$




$$
\boldsymbol{I}_{m}(\lambda)=\sum_{n=1}^{i}\left[C_{n} \boldsymbol{A}_{n}(\lambda)+\boldsymbol{B}_{n}(\lambda)\right]
$$

\section{Globally Optimal Peak Combination}

In addition to selecting peaks based on our criteria, concentration estimates were also calculated for every combination of peaks in parallel and perpendicular polarizations to find a globally optimal combination that yields an even lower RMSE. By comparing this to the combination of peaks identified based on sensitivity using our method, the peak selection criteria we developed would be validated if there exists good agreement between the two sets of combinations.

\subsection{Results and Discussion}

\section{Protein Interactions}

Predictions of the mixture spectra were carried out before and after background subtraction to investigate the effect of potential protein-protein interactions as well as to provide a visual sense of how well the mixture spectra were fit by the basis spectra. The predicted spectra were created by calculating the slope (intensity/concentration) and intercept (intensity) at each wavenumber of the component spectra of both proteins. The proteins' predicted intensity, at each wavenumber, was then found by multiplying the slope by the desired concentration and adding the intercept value, after which the predicted intensity values of each protein were summed to reconstruct each mixture spectrum.

The chances of physical protein-protein interactions, especially within the short time frame of this experiment (each mixture waited no more than 15 minutes before Raman detection), were slim due to the slight positive charge of amylase 
and strong positive charge of lysozyme leading to mutual electrostatic repulsion. Amylase has a reported isoelectric point ${ }^{137}$ of 7.18 , and lysozyme has an isoelectric point ${ }^{138}$ of 11.35 . Furthermore, once dissolved, the protein mixtures formed a clear solution showing no sign of aggregation or precipitation. It is worth noting, as seen in Figure 3.4, Figure 3.5, and Figure 3.6 (corresponding to the parallel, perpendicular, and traditional RS detection geometries, respectively), that the background fluorescence of the mixtures were not the mere sum of the two proteins. This observation was seen in all 25 mixture combinations (data not shown). Instead, the measured spectra displayed considerably less background fluorescence than the calculated sum of fluorescence from both proteins. After background subtraction, a significant improvement in agreement between the measured and reconstructed spectra was found. This result implies some sort of increased absorption of fluorescent light caused by the mixture. However, the Raman peaks still seemed to be the sum of both protein Raman signals. Another possible explanation is related to the general rule of scattering which states that denser substances decrease lateral scattering due to more complete destructive interference. ${ }^{139}$ In this case, the presence of both proteins may have constituted a relatively denser, more uniform, and ordered medium to cause more complete lateral destructive interference. ${ }^{139}$ But scattered Raman light would have been equally affected, which was not observed. 

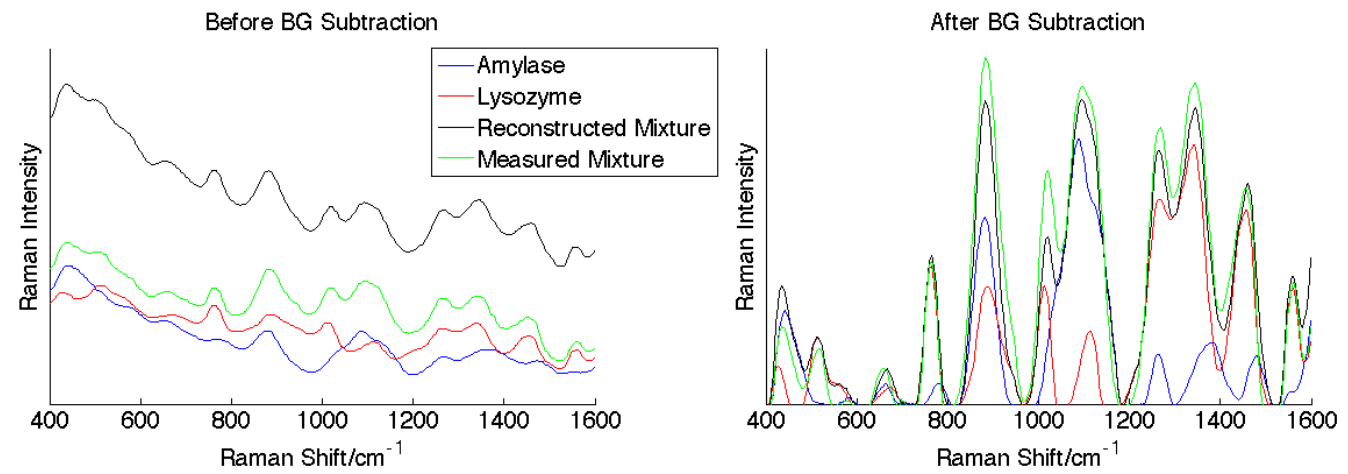

Figure 3.4 Comparison of spectra before (left) and after (right) background removal. Component spectra of amylase (blue) and lysozyme (red) measured in the parallel configuration at $50 \mathrm{~g} \mathrm{~L}^{-1}$ for both proteins. Reconstructed spectrum (black), based on calculation, of amylase and lysozyme mixture with concentrations of 39 and $48 \mathrm{~g} \mathrm{~L}^{-1}$, respectively. Measured spectra (green) of amylase and lysozyme mixture with concentrations of 39 and $48 \mathrm{~g} \mathrm{~L}^{-1}$, respectively.

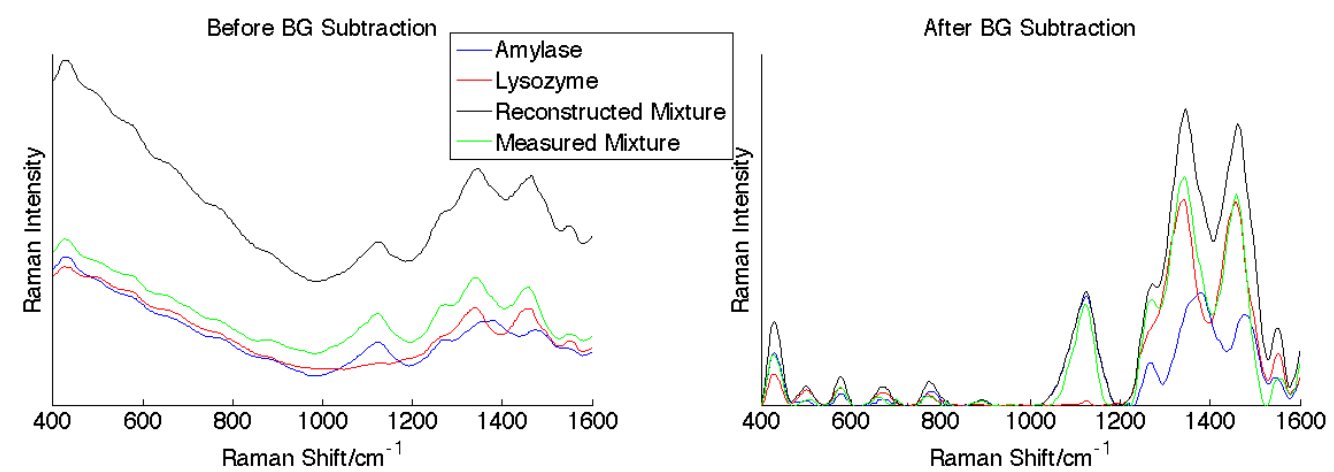

Figure 3.5 Comparison of spectra before (left) and after (right) background removal. Component spectra of amylase (blue) and lysozyme (red) measured in the perpendicular configuration at $50 \mathrm{~g} \mathrm{~L}^{-1}$ for both proteins. Reconstructed spectrum (black), based on calculation, of amylase and lysozyme mixture with concentrations of 39 and $48 \mathrm{~g} \mathrm{~L}^{-1}$, respectively. Measured spectra (green) of amylase and lysozyme mixture with concentrations of 39 and $48 \mathrm{~g} \mathrm{~L}^{-1}$, respectively. 

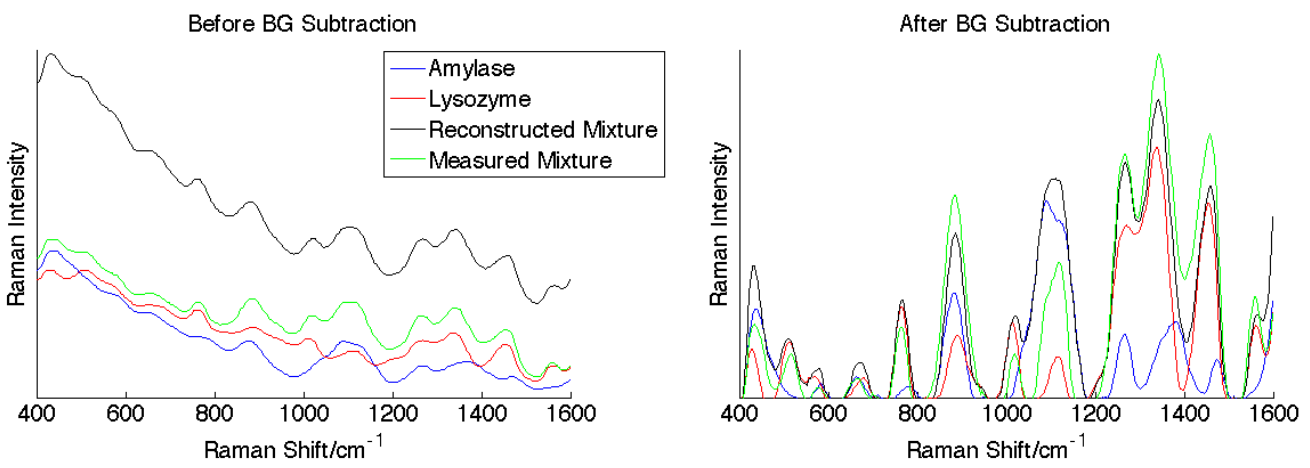

Figure 3.6 Comparison of spectra before (left) and after (right) background removal. Component spectra of amylase (blue) and lysozyme (red) measured in the traditional RS configuration at $50 \mathrm{~g} \mathrm{~L}^{-1}$ for both proteins. Reconstructed spectrum (black), based on calculation, of amylase and lysozyme mixture with concentrations of 39 and $48 \mathrm{~g} \mathrm{~L}^{-1}$, respectively. Measured spectra (green) of amylase and lysozyme mixture with concentrations of 39 and $48 \mathrm{~g} \mathrm{~L}^{-1}$, respectively. 

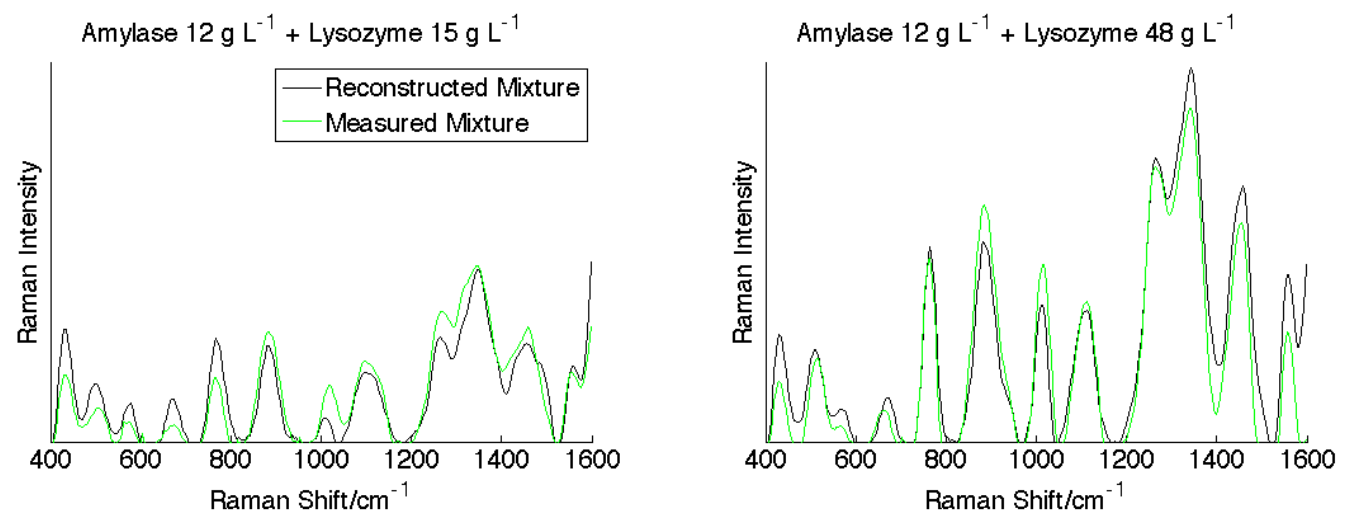

Figure 3.7 Comparison of reconstructed and measured mixture spectra with amylase held constant at its lowest concentration and lysozyme shown at its lowest and highest concentrations. These spectra correspond to the parallel detection geometry.
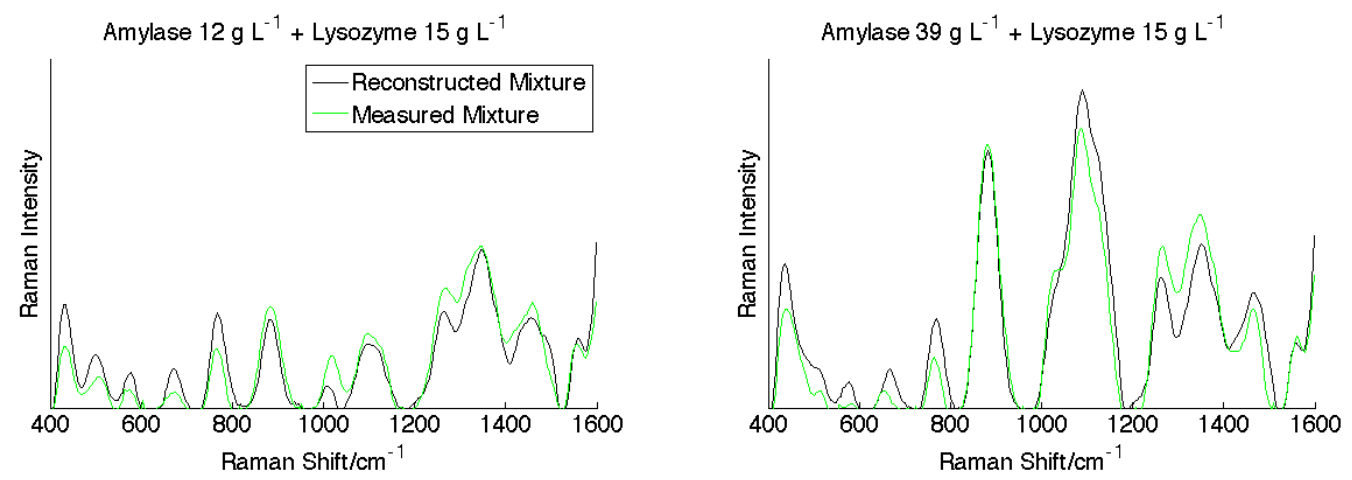

Figure 3.8 Comparison of reconstructed and measured mixture spectra with lysozyme held at its constant lowest concentration and amylase shown at its lowest and highest concentrations. These spectra correspond to the parallel detection geometry. 
An analysis of the reconstruction accuracy as the concentration of one protein was increased while the other was held constant was explored to seek further evidence of possible protein-protein interaction. Figure 3.7 and Figure 3.8 provide a rough visual sense of how the reconstruction accuracy decreases as the concentration of one component was raised. In Figure 3.7, amylase was held at its lowest concentration of $12 \mathrm{~g} \mathrm{~L}^{-1}$ and the measured and reconstructed mixture spectra are compared between lysozyme at its lowest and highest concentrations of 15 and $48 \mathrm{~g} \mathrm{~L}^{-1}$. Similarly, the same was done in Figure 3.8 only the concentration of lysozyme was held constant instead. The accuracy of the reconstruction was then calculated as the root mean squared error (RMSE) for each mixture combination and polarization detection geometry, and are summarized in Table 3.3, Table 3.4, and Table 3.5.

Table 3.3 Summary of the root mean squared error between measured and reconstructed spectra for each concentration combination measured in the parallel detection geometry.

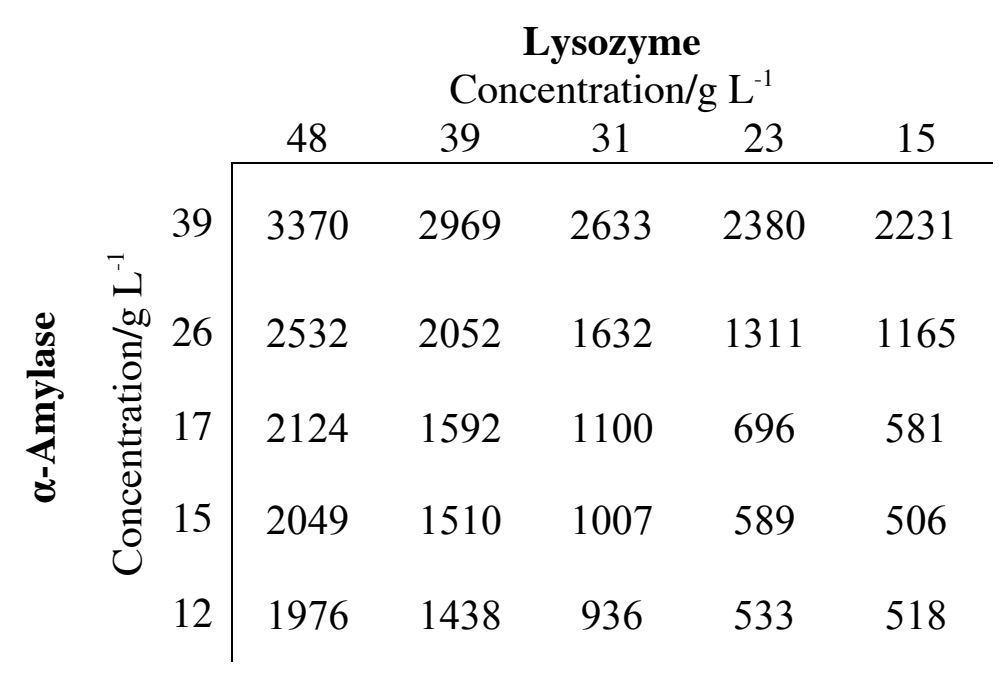


Table 3.4 Summary of the root mean square error between measured and reconstructed spectra for each concentration combination measured in the perpendicular detection geometry.

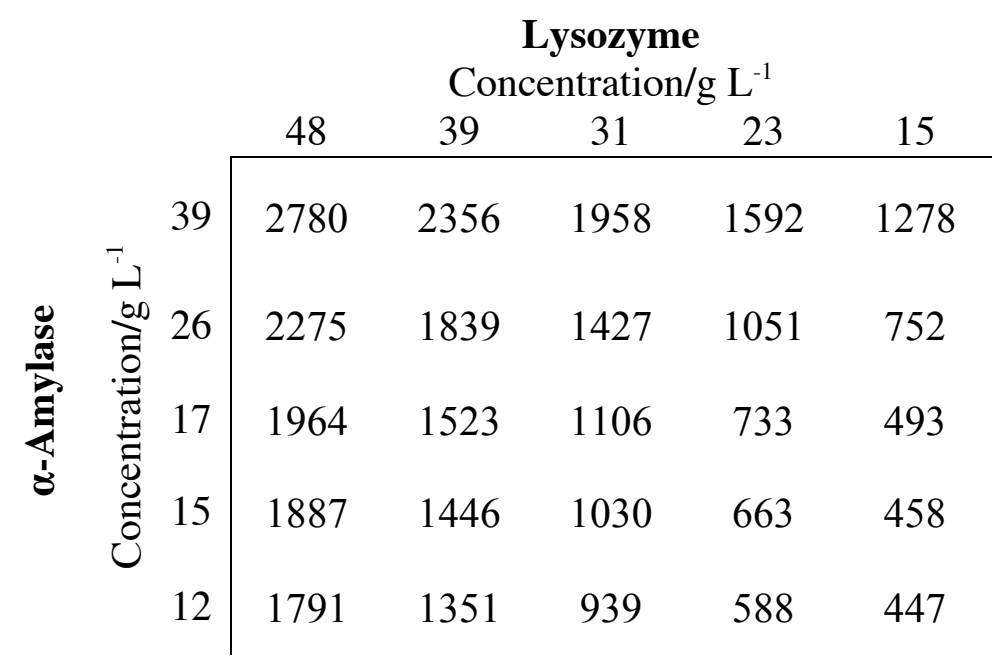

Table 3.5 Summary of the root mean square error between measured and reconstructed spectra for each concentration combination measured in the unpolarized detection geometry.

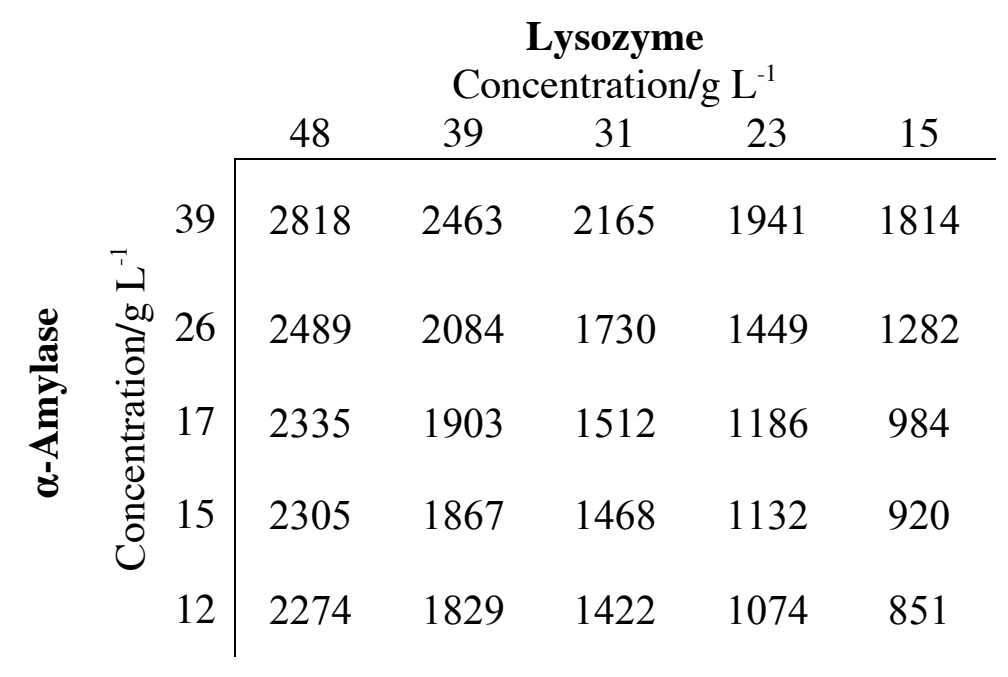


Analysis of the RMSE as a function of concentration revealed a clear and consistent trend in which the reconstruction accuracy decreased with increasing concentration. However, taking a closer look at the background removal method, it was found to have more difficulty with spectra containing taller peaks, corresponding to higher concentrations. To illustrate this point, the raw spectra with their corresponding baseline fluorescence are shown in Figure 3.9. From top to bottom, each spectrum corresponds to a mixture listed on the diagonal of Table 3.1 from the top left to bottom right. Particularly at the peaks between 1000 and $1500 \mathrm{~cm}^{-1}$, the wavelet algorithm had more difficulty reaching the base of these peaks at higher levels of background fluorescence. Conversely, more of the fluorescence could be removed as the concentration decreased. Given that the fluorescent background significantly decreased reconstruction accuracy, and, by extension, would ultimately affect concentration estimates, its large remanences at high concentration is what likely caused the RMSE to worsen. 


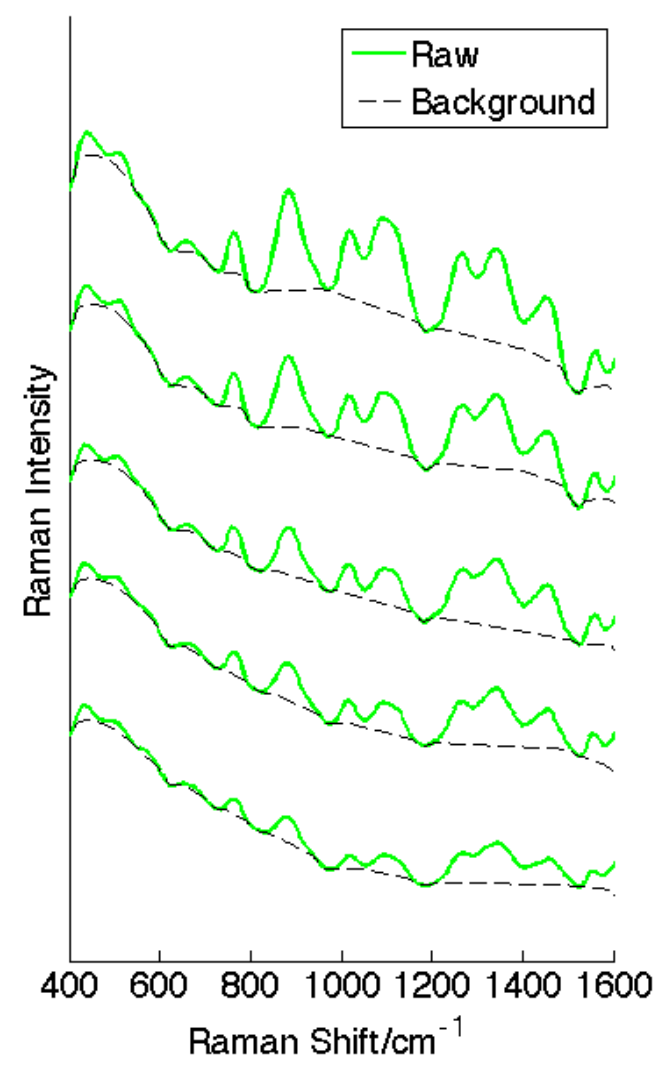

Figure 3.9 Illustration of the decrease in background fluorescence removal performance as a function of overall protein concentration. From top to bottom, the mixture concentrations for amylase and lysozyme are, respectively, 39/48, 26/39, 17/31, 15/23, and 12/15; all are in units of $g \mathrm{~L}^{-1}$.

These results suggest that the two proteins did not physically interact. However, a determination of what caused the non-additive nature of the background fluorescence requires further investigation that is beyond this study's purview. For our purpose here, background fluorescence removal was the most sensible solution to maximize the reconstruction accuracy; otherwise the model, shown in Eq. 2 and Eq. 3, would no longer be valid. Concentration estimation was attempted on raw spectra with the background included, but yielded nonsensical results such as negative concentrations. Although the mixture spectra could not be perfectly modeled after background subtraction, it was not significant enough to invalidate the comparison between RS and PRS. 


\section{Polarized and Traditional Raman Spectra Comparability}

In order to minimize the effect of noise to ensure fair comparison between these two methods, namely polarized and traditional RS, exposure times were chosen to maximize the Raman signals without saturating the detector when taking both sets of spectra. In this study, 12 seconds yielded the strongest Raman peaks without saturation during traditional RS measurements. Consequently, addition of the analyzer weakened the overall signal, which called for an increase in exposure time to 22 seconds for polarized RS measurements. 

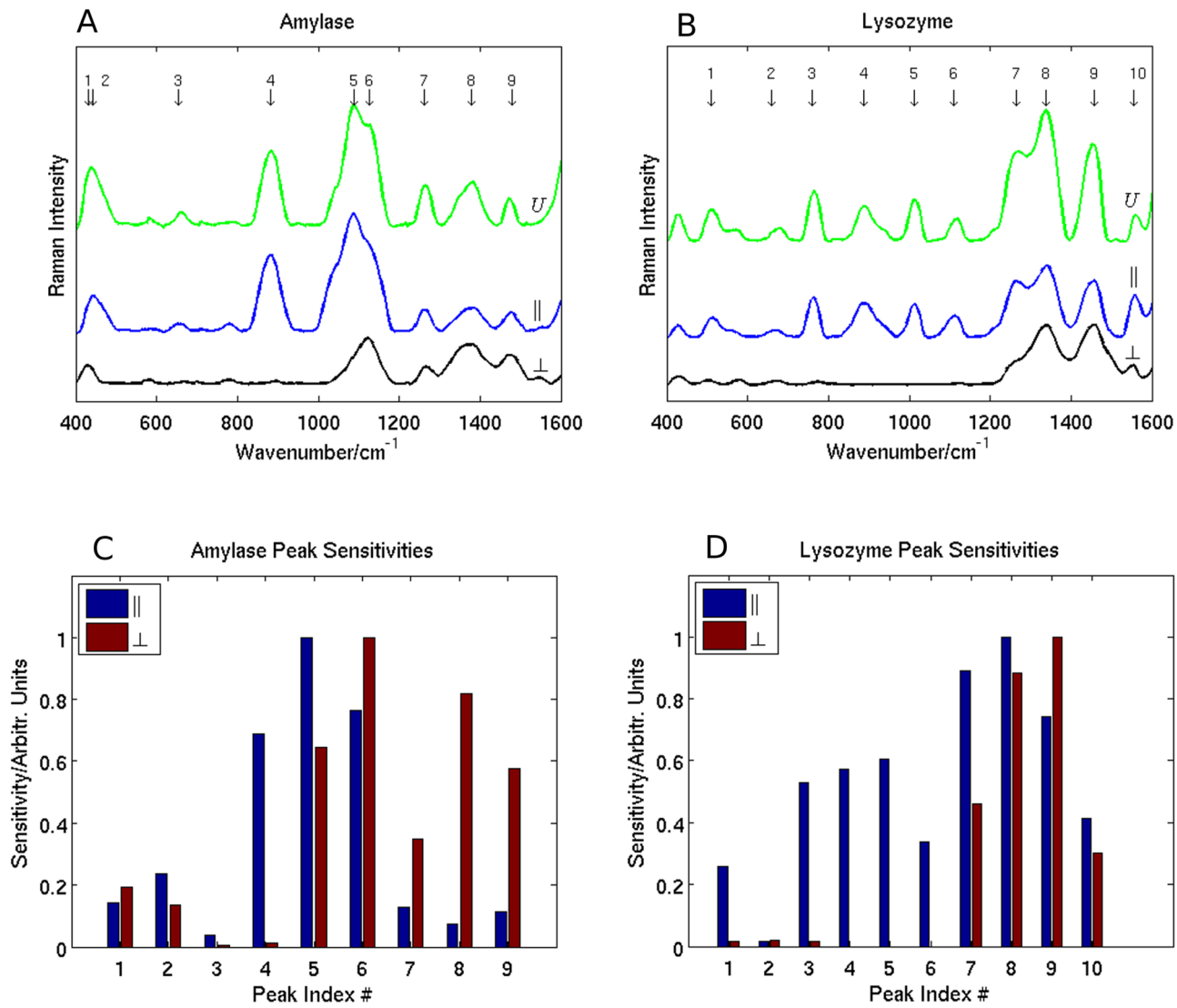

Figure 3.10 Basic component spectra of (A) $\alpha$-amylase and (B) lysozyme. Numbers above the arrows represent the peak index number. Arrows mark the chosen peaks for concentration estimation. From top to bottom of (A) and (B): (U) unpolarized spectra from traditional RS setup, (II) parallel and $(\perp)$ perpendicular spectra. The sensitivities of these peaks are plotted for (C) $\alpha$-amylase and (D) lysozyme.

To further justify the fairness of this comparison, we analyzed the signal-tonoise ratio (SNR) of all basic component spectra, which was defined as the ratio of the mean value of a Raman peak to its standard deviation calculated from multiple repeated measurements. The SNR (data not shown) of all major peaks (4, 7, 8 and 9 of $\alpha$-amylase and $1,3,4,5,6,7,9$ and 10 of lysozyme) were comparable between polarized and traditional spectra. Moreover, most major peaks had an SNR of 10 or greater for both methods making them sufficiently strong to minimize the influence of noise on the final concentration estimations. 


\section{Criteria Derived Peak Combinations}

Sensitive peaks, selected based on our criteria, from the array of PRS data were used for selective concentration estimation of lysozyme and $\alpha$-amylase in a mixed solution. The accuracy of these estimates were characterized by the RMSE and compared to that obtained using data from standard RS.

Spectra obtained from $\alpha$-amylase and lysozyme shown in Figures $4.10 \mathrm{~A}$ and 4.10B contained an array of peaks from which most were used for NLLSR. The first peak of lysozyme located after $400 \mathrm{~cm}^{-1}$ was ignored because its intensity lacked correlation to change in the corresponding protein's concentration. Difficulty in accurate background removal due to the first peak's relatively small magnitude is what likely rendered it unusable. 
Table 3.6 Combinations of peak polarizations chosen for $\alpha$-amylase and lysozyme

$$
\begin{aligned}
& \begin{array}{ll|lllllllll}
\multicolumn{10}{c}{} & \multicolumn{10}{c}{\text { Peak Index \# }} \\
\cline { 3 - 10 } \alpha \text {-Amylase } & & & 2 & 3 & 4 & 5 & & & & \\
& \perp & 1 & & & & & 6 & 7 & 8 & 9
\end{array} \\
& \text { Peak Index \# } \\
& \begin{array}{ll|lllllllllll}
\cline { 3 - 5 } \text { Lysozyme } & \| & 1 & & 3 & 4 & 5 & 6 & 7 & 8 & & 10 \\
& \perp & & 2 & & & & & & & 9 &
\end{array}
\end{aligned}
$$

Further evaluation of sensitivity given in Figures $4.10 \mathrm{C}$ and $4.10 \mathrm{D}$ provided a new combination for polarizations given in Table 3.6. A comparison of concentration estimates using RS and PRS is given in Figure 3.11. Excellent estimates from the PRS method compared to RS are consistent with our premise that peaks with greater sensitivity improves accuracy and reproducibility of NLLSR. The root mean squared error of $\alpha$-amylase decreased from $6.7 \mathrm{~g} \mathrm{~L}^{-1}$ to $1.9 \mathrm{~g} \mathrm{~L}^{-1}$, and that of lysozyme decreased from $4.2 \mathrm{~g}$ $\mathrm{L}^{-1}$ to $1.6 \mathrm{~g} \mathrm{~L}^{-1}$ when the data used for estimation was switched from RS to PRS, respectively. Table 3.7 summarizes the RMSE achieved for each explored method.

Table 3.7 The root mean square error of concentration estimations for $\alpha$-amylase and lysozyme using traditional RS, PRS with our selection criteria and the globally optimal combination

\begin{tabular}{rcc} 
& \multicolumn{2}{c}{$\mathrm{RMSE} / \mathrm{g} \mathrm{L}^{-1}$} \\
\cline { 2 - 3 } Conventional RS & $\alpha$-Amylase & Lysozyme \\
\cline { 2 - 3 } PRS Selection Criteria & 1.9 & 4.2 \\
PRS Optimum Combination & 0.6 & 1.6 \\
&
\end{tabular}

The RMSE of RS and PRS were also compared using similar exposure times to show that the improvement in concentration estimation was primarily due to our selection criteria rather than the longer exposure time used in PRS measurements. For RS, two unpolarized spectra, which were obtained using a total integration time of 24 seconds, were averaged and input into the 
algorithm. For PRS, one spectrum with a combination of peaks from both the parallel and perpendicular spectra, which contains the same number of data points as either one of the polarized spectra thus involving an equivalent integration time of 22 seconds, was used as the input. The averaged RMSE of amylase and lysozyme for PRS spectra were 3.0 and 2.6, respectively. In contrast, the averaged RMSE of amylase and lysozyme for the RS spectra were 5.4 and 3.9, respectively. Given this result, the PRS using the proposed selection criteria generated lower RMSEs compared to traditional RS when the integration times were nearly equal. This observation clearly indicates that PRS shows the advantage in estimation accuracy over RS even in the case of comparable integration times.
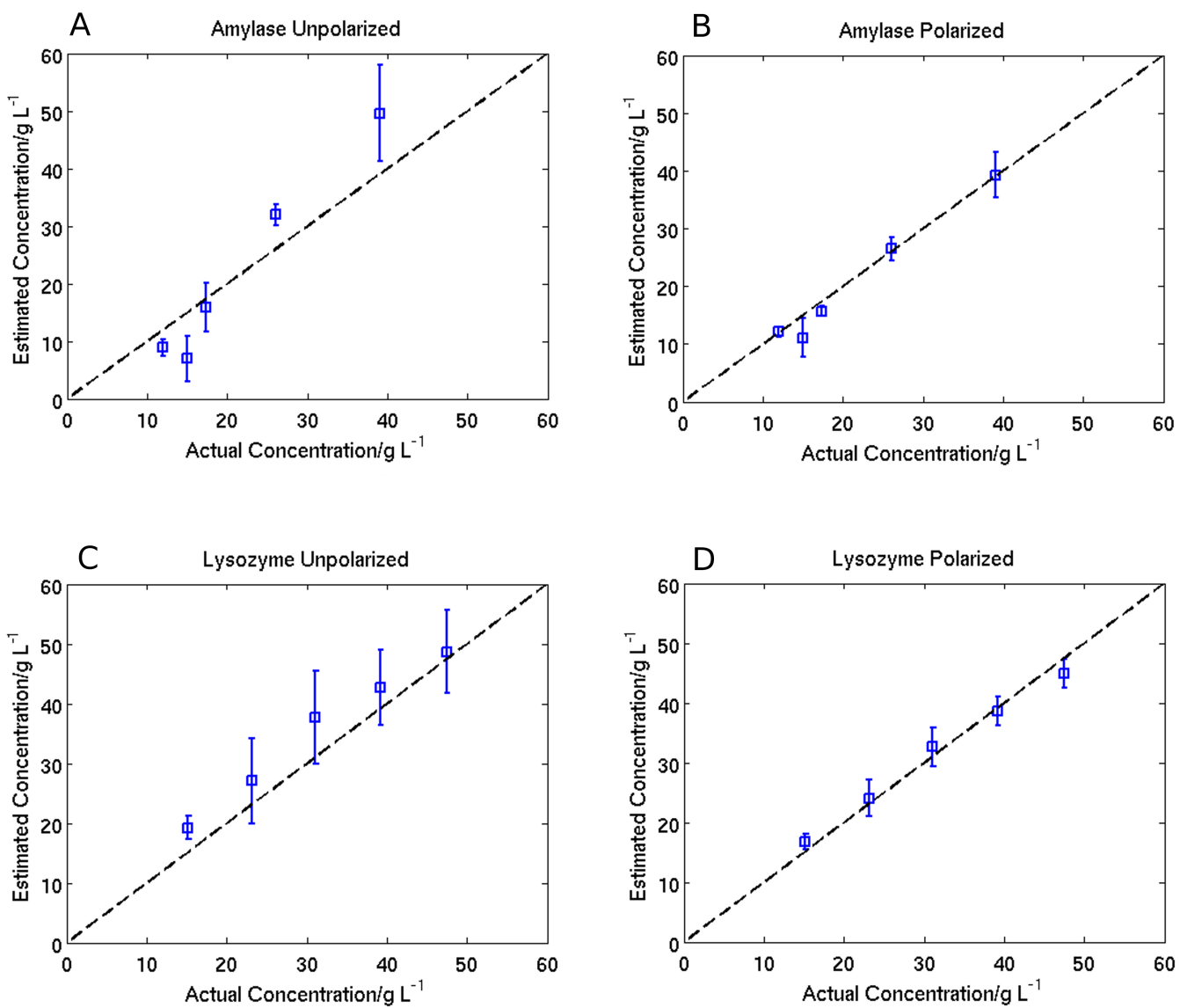

Figure 3.11 Concentrations estimated using traditional RS (A and C) and PRS (B and D) 


\section{Prediction Bias}

Correlation between the predicted concentrations of the first protein and actual concentration of the second protein was investigated. The concentration estimates derived from traditional RS appear to contain some bias and may imply some dependence of the amylase prediction on the amount of lysozyme and vice versa. The predicted concentration values were plotted against the known concentration of the other protein for RS and PRS in Figure 3.12 and Figure 3.13 , respectively. Since it is difficult to discern a correlation by mere visual inspection of these figures, the correlation coefficient (Spearman's correlation coefficient) was calculated for each predicted concentration value and summarized in Table 3.8 for both RS and PRS.
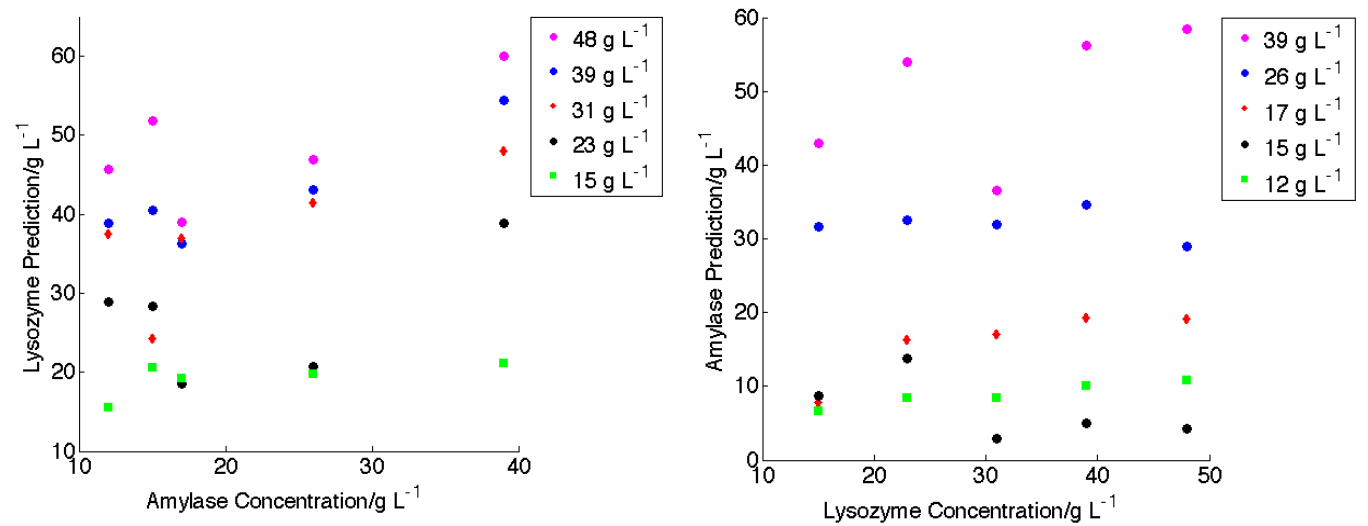

Figure 3.12 Correlation between predicted concentrations of the first protein versus the actual concentration of the second protein measured with traditional RS. Left: predicted lysozyme concentrations versus the known concentrations of amylase. Right: predicted amylase concentrations versus the known concentrations of lysozyme. The legends indicate which markers correspond to which predicted concentration. 

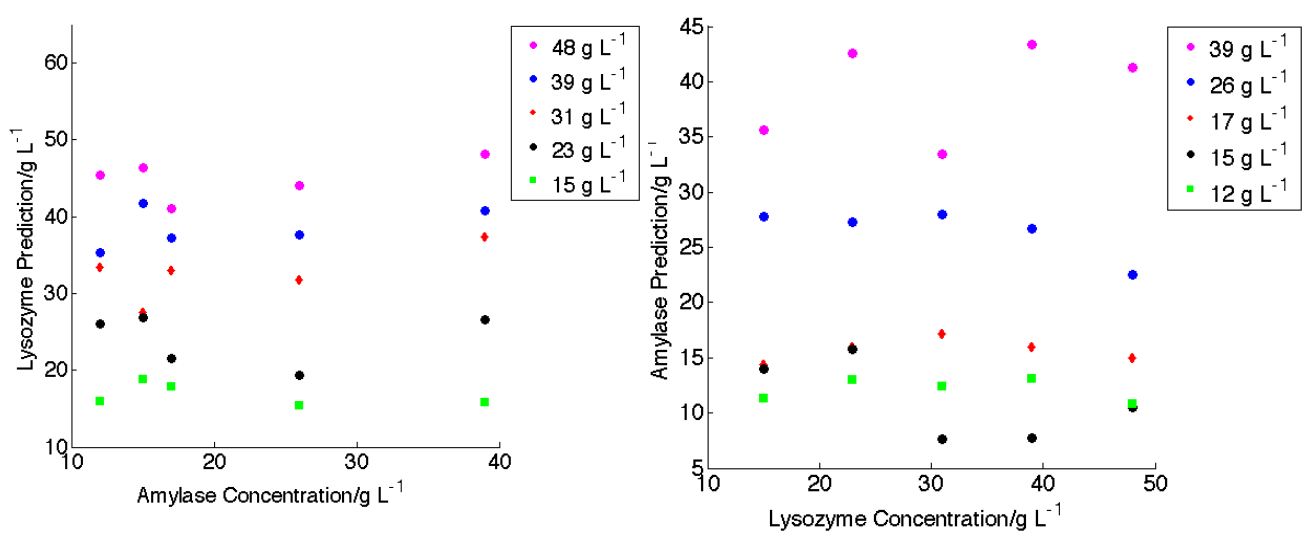

Figure 3.13 Correlation between predicted concentrations of the first protein versus the actual concentration of the second protein measured with PRS. Left: predicted lysozyme concentrations versus the known concentrations of amylase. Right: predicted amylase concentrations versus the known concentrations of lysozyme. The legends indicate which markers correspond to which predicted concentration.

Table 3.8 Summary of the correlation coefficient for each predicted concentration and the corresponding concentration of the second protein. The correlation coefficient for RS and PRS are both shown. Relatively high correlation coefficient values (close to 1) were highlighted in yellow. Values that increased when switching to PRS were highlighted in red.

\begin{tabular}{|c|c|c|c|c|c|}
\cline { 2 - 5 } \multicolumn{1}{c|}{} & \multicolumn{2}{c|}{$\begin{array}{c}\text { Correlation } \\
\text { Coeff. }\end{array}$} & \multicolumn{2}{c|}{$\begin{array}{c}\text { Correlation } \\
\text { Coeff. }\end{array}$} \\
\hline Amylase $\left(\mathrm{g} \mathrm{L}^{-1}\right.$ ) & RS & PRS & Lysozyme $\left(\mathrm{g} \mathrm{L}^{-1}\right)$ & RS & PRS \\
\hline 39 & 0.7 & 0.3 & 48 & 0.5 & 0.2 \\
\hline 26 & -0.1 & -0.7 & 39 & 0.7 & 0.4 \\
\hline 17 & 0.9 & 0.3 & 31 & 0.7 & 0.3 \\
\hline 15 & -0.6 & -0.5 & 23 & 0.1 & -0.2 \\
\hline 12 & 1 & -0.1 & 15 & 0.7 & -0.06 \\
\hline
\end{tabular}


On the whole, relatively high correlation coefficient values (close to 1) were found for some concentrations, and were highlighted in Table 3.8. This suggests the existence of some bias when using RS. These values, with exception of the two highlighted in red, decreased after switching to PRS, which implies that using PRS can decrease the concentration prediction dependency on the other protein's amount. This may likely be another factor behind the enhanced accuracy observed when using PRS.

\section{Globally Optimal Combination}

The globally optimal combination was obtained for comparison to the combination obtained using our sensitivity method. The globally optimal combination of polarized bands was determined, given by Table 3.9, after testing every possible combination to find which provided the lowest RMSE. Asterisks (*) indicate were the polarization was chosen opposite to that of the PRS results. A lower limit of $0.6 \mathrm{~g} \mathrm{~L}^{-1}$ was achieved for both proteins as shown earlier in Table 3.7. Figure 3.14 illustrates the concentration estimates using the globally optimal combination.

Table 3.9 Globally optimal combination of polarization peaks that yielded the lowest RMSE. Asterisks (*) indicate were the polarization was chosen opposite to that of the PRS results.

\begin{tabular}{|c|c|c|c|c|c|c|c|c|c|c|c|c|}
\hline \multirow{3}{*}{$\alpha$-Amylase } & & \multicolumn{11}{|c|}{ Peak Index \# } \\
\hline & $\|$ & & & 2 & $*$ & 4 & * & & & & & \\
\hline & $\perp$ & & 1 & & 3 & & 5 & 6 & 7 & & & 9 \\
\hline & & & & & & Peak & Inde & $\mathrm{x} \#$ & & & & \\
\hline Ime & & $*$ & 2 & * & & 5 & 6 & 7 & & 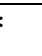 & & $*$ \\
\hline Lysozyme & & 1 & * & 3 & & & & & & 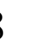 & 9 & 10 \\
\hline
\end{tabular}



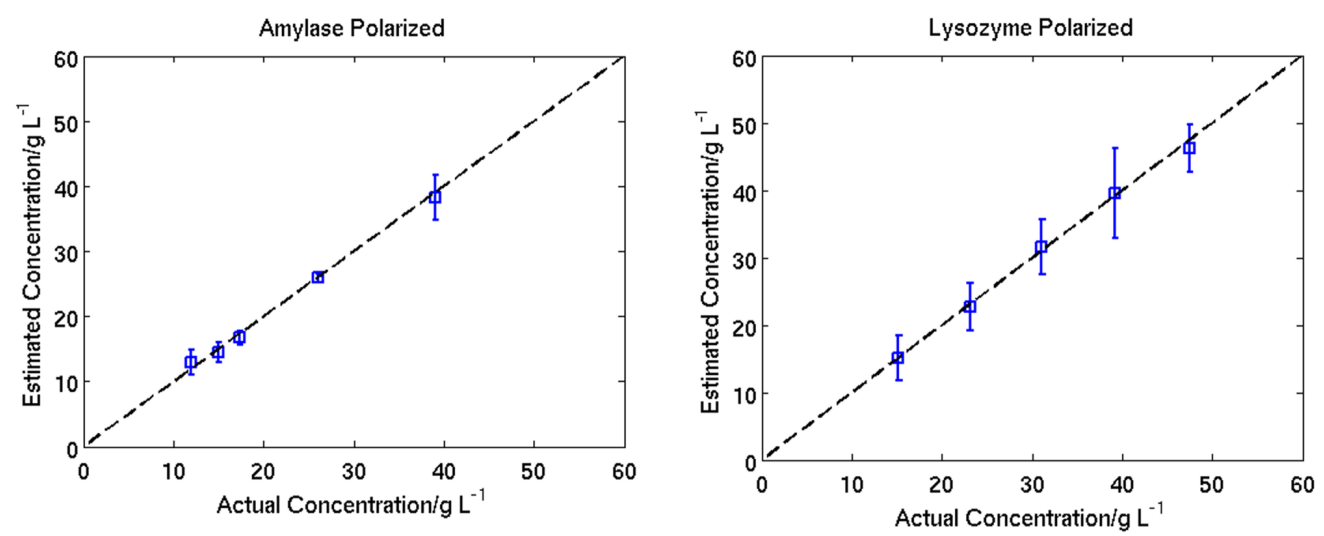

Figure 3.14 Concentrations estimated using the globally optimal combination

For amylase, the globally optimal combination is, for the most part, consistent with the optimal combination found using the sensitivity selection criteria described earlier, which indicates good effectiveness of the criteria. For lysozyme, only half (5 out of 10$)$ of the combinations agreed. In the case of lysozyme, peak 2 was chosen in the parallel polarization. Additionally, peaks 1, 3,8 and 10 were selected in the perpendicular polarization where peaks 1,3 , and 8 nearly vanished, and peak 10 reduced in magnitude. Switching to less intense peaks may have facilitated the background removal algorithm, which was shown earlier to have more difficulty at high peak intensities. As a result, switching to perpendicular polarization enabled more complete background subtraction so that less fluorescent background was inputted, and may have led to improved estimation accuracy. Another factor potentially creating these exceptions is the robustness of the background removal method to faithfully preserve peak height. For example, peak 3 of $\alpha$-amylase and peak 10 of lysozyme were exceptions likely due to their relatively short heights making them more prone to the influence of imperfect background removal. 


\section{Random Peak Comparison}

Given the discrepancy between the proposed method and the globally optimal combination, we compared the RMSE of the peak combination selected using the proposed criteria to those of 20 randomly generated combinations with the purpose of demonstrating that our method can reliably yield more accurate results than any randomly assembled combination of polarized bands. With the help of a random number generator, 20 randomly selected combinations were generated and divided evenly between the two proteins to give a total of ten trials for each protein. The RMSE for each trial was determined and shown in Table 3.10. In all ten trials, the RMSEs never fell below those obtained using our selection criteria, which substantiated the proposed method and provided assurance that it can generate more accurate estimates than a randomly chosen combination for the vast majority of the time.

Table 3.10 The root mean square error of randomly generated peak combinations

\begin{tabular}{|c|c|c|c|c|c|c|c|c|c|c|c|}
\hline & & \multicolumn{10}{|c|}{ Trial \# } \\
\hline & & 1 & 2 & 3 & 4 & 5 & 6 & 7 & 8 & 9 & 10 \\
\hline Amylase & 岕 & 4.3 & 2.9 & 2.0 & 4.9 & 2.5 & 7.0 & 3.1 & 3.0 & 2.5 & 2.0 \\
\hline Lysozyme & $\sum_{\propto}$ & 2.5 & 4.1 & 4.0 & 2.6 & 3.2 & 2.1 & 3.9 & 2.5 & 4.4 & 1.9 \\
\hline
\end{tabular}

\subsection{Conclusion}

Polarized Raman spectroscopy (PRS) was explored to enhance the quantitative accuracy of an aqueous mixture of $\alpha$-amylase and lysozyme. Raman peaks exhibiting greater sensitivity to change in their associated protein's concentration when viewed in the parallel or perpendicular configuration were used in non-linear least squares regression to estimate concentrations. In comparison to the concentration estimates derived from a conventional Raman spectroscopy configuration in which there is no differentiation in detected Raman polarization, results from our method using PRS showed significant 
improvement in quantification accuracy and reproducibility as reflected by the RMSE and standard deviation.

All possible combinations of peaks comprised of the parallel and/or perpendicular polarizations were also explored, and revealed a globally optimal combination that resulted in significant improvement of quantification accuracy in comparison to the two previous approaches. Evaluating all combinations had the unique advantage of minimizing data that was detrimental to the performance of protein quantification, which effectively neutralized other factors from compromising the quantitative enhancing power of peaks with greatest sensitivity. Lastly, our method was further substantiated when compared to the results of randomly generated combinations that consistently yielded lower accuracy.

Applying the proposed method to more complex protein mixtures containing more than two proteins would be possible since peak selection is based on the individual basic component spectra of each protein comprising the mixture. Bands would be chosen in the same way as shown for $\alpha$-amylase and lysozyme and applied in the same way to quantify the final mixture. However, in a multivariate system, the Raman photons may not add linearly, and require a more sophisticated prediction model.

As this study provided compelling evidence showing improved quantitative performance of this method, PRS has great promise to play a key role in future detection platforms for biological analyte quantification. If combined with Raman enhancement methods to detect proteins on a biological scale, such as SERS or CARS, PRS may be able to achieve a better detection limit and 
become a highly desirable technique in applications requiring cost effective and fast protein monitoring, such as tissue engineering, as an alternative to current protein detection methods such as Western blotting and enzyme linked immunosorbent assays. 


\section{Chapter 4 Surface Enhanced Raman Optical Activity for Detection and Quantification of Aqueous Protein Mixtures}

\subsection{Introduction}

Proteins are chemically identical by and large due to the finite number of existing amino acids and prosaic recurrence of amide bonds. Hence, scrutiny of their chemical makeup leads to a representation ill-suited for distinguishing different protein species and alternate morphologies of the same protein. To overcome this obstacle, attention must be shifted to structure - a trait that typifies a protein's role and behavior in living systems. Surface enhanced Raman optical activity (SEROA) is potentially well suited for this application as it can access structural information to enable the identification of individual protein species within mixtures qualitatively and quantitatively within minutes as opposed to several hours or days using state of the art methods such as Western blotting and ELISA. As formerly mentioned, ROA is an extremely weak phenomenon that would profoundly benefit from the dramatic enhancement of SERS to access low concentrations found in biological systems. ${ }^{140}$ Additionally, potentially damaging high laser power levels and long exposure times would be significantly reduced. Since the first report of ROA in 1973 by Barron et. al. ${ }^{141}$, only a handful of SEROA studies exist ${ }^{6,21,140,142-144}$ likely due to the rich challenges it poses. The most prominent challenge is the minimization of spectral artifacts that poses an immensely difficult task given the requirement of micron-precision spatial alignment of optical components as well as components of premium quality to reduce any unwanted stray birefringence. $^{24}$ More importantly is the optically altering effect of nanoparticles whereby the polarization state of the exciting beam is not 
necessarily maintained by the locally enhanced electromagnetic field sensed by the analytes. ${ }^{145}$

This project attempts to measure the SEROA spectrum of several individual proteins to help deconvolute their collective Raman signal when mixed in solution.

The following sections will describe the process of constructing and calibrating the ROA setup, preliminary results, a brief discussion, and future steps required to complete this project.

\subsection{Construction and Calibration of ROA optical setup}

A wide variety of obstacles arose throughout the course of construction and calibration, which will be discussed chronologically in the order they were dealt with.

\subsubsection{SCP-ROA}

Three optical schemes were explored, namely: SCP-ROA, ICP-ROA, and DCPI-ROA. The initial choice was SCP-ROA, shown schematically in Figure 4.1 with the exception of a linear polarizer in place of the polarized beam splitting cube (Figure 4.1A), since no circular polarization (CP) of the incident beam was necessary. Please note that the fiber launch, labeled as FL, describes a lens focusing the scattered radiation into a multimode fiber. Samples were excited by a $785 \mathrm{~nm}$ laser (Toptica XTRA II) with a power of about $100 \mathrm{~mW}$. Note: this laser wavelength was not ideal for this study due to a limitation of resources. Raman scattered light was detected using a Princeton Instruments Acton LS 785 spectrometer optimized for ultra-high sensitivity in the near-IR range from $750-1100 \mathrm{~nm}$. Scattered RCP and LCP light were selected by a $90^{\circ}$ 
rotation of the analyzer after conversion to linear polarization (LP) by a quarterwave plate (QWP). Although the ideal molecule of our first attempt at measuring ROA would either be achiral or have a known ROA spectrum, we began with measuring the SEROA of lysozyme using spherical AgNPs synthesized using the Lee and Miesel method. ${ }^{146}$ Details of AgNP synthesis and the flocculation procedure are provided in the following sections. Aggregated samples were placed within a $200 \mu \mathrm{L}$ well in a shallow 96-well plate. The laser spot was positioned in the middle of this volume to the best of our ability. Predictably, the result was incorrect for a variety of reasons, but the experiment revealed one major issue: the inherent instability of aggregated nanoparticles leading to erratic SERS signals. Subsequently, the SERS signal subsided entirely as all aggregates sank to the well's base. As a consequence, peak intensity and the overall baseline of the spectrum displayed erratic fluctuations throughout the exposure time. Since RCP and LCP light were measured in series, it was impossible to derive a pure SEROA spectrum since both component spectra contained a different history of fluctuations. Furthermore, as aggregates sank due to gravity, the signal intensity was noticeably weaker during the second exposure as increasingly fewer aggregates were present within the laser focal volume. 


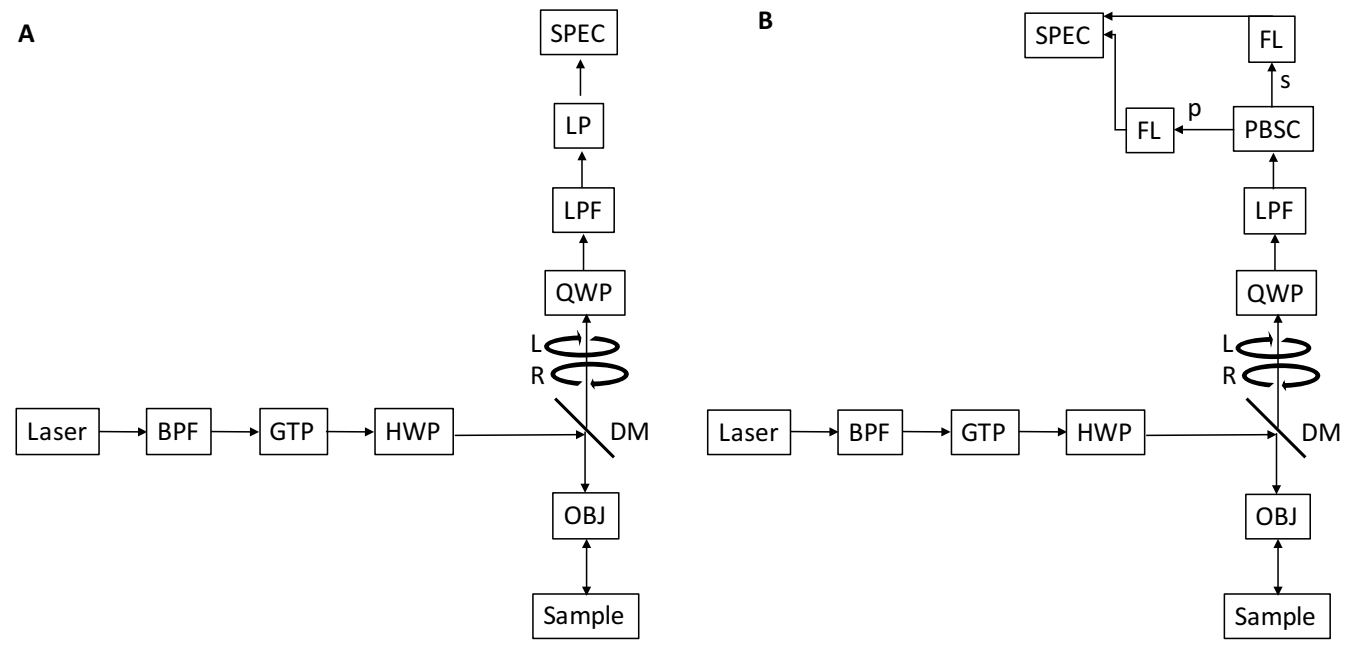

Figure 4.1 SCP-ROA optical diagram with a linear polarizer (LP) (A) and with polarized beam splitting cube (PBCS) (B). Band-pass filter (BPF), Glan-Taylor polarizer (GTP), half-wave plate (HWP), objective lens (OBJ), dichroic mirror (DM), quarter-wave plate (QWP), long-pass filter (LPF), fiber launch (FL) for s- and p-polarized states, and spectrograph (SPEC). 
A straightforward solution was to install a polarized beam splitting cube (PBSC) in lieu of the analyzer as seen in Figure 4.1B. This enabled simultaneous measurement of scattered RCP and LCP light of the same laser focal volume by means of a bifurcated fiber that collected light from both branches of the PBSC and imaged both channels onto the CCD concurrently. If successful, these fluctuations as well as any other transient fluctuations within the sample volume would cancel upon subtraction after having calibrated for the transmittance difference between the two channels. ${ }^{6}$ Interestingly, the fluctuations differed significantly where the decrease in fluorescence baseline in one channel would cause an increase in the other and vice versa. This may be attributed to the locally enhanced EM field polarization dependence on the angle of the excitation beam relative to the aggregates' orientation ${ }^{142,145,147}$ and other unknown factors. Although we could have sought ways to stabilize the aggregates, there was a much more fundamental problem of inadmissible crosstalk between the two channels. Due to optical aberration known as field astigmatism inherent to Czerny-Turner spectrometers, the slit images of each channel became broad and distorted towards the left and right ends of the CCD (along the wavelength axis), which is sometimes known as the "bow tie" effect. ${ }^{148}$ In the center, however, the channels' images were thin, straight, and clearly resolved. ${ }^{148}$ The resulting overlap made subtraction impossible to calculate the ROA signal except for within a the narrow range of wavenumbers in the center. As a result, this approach was ultimately discontinued since we were forced to omit the majority of spectral data and sacrifice vast amounts of invaluable structural information.

However, before switching to another approach, we still attempted to measure the ROA of urea and chloroform within the narrow spectral window. 
Unfortunately, all results were erroneous in large part due to uncorrected spectral artifacts. A major artifact was caused by the impossibility of aligning the excitation beam plane of polarization to be precisely $45^{\circ}$ relative to the fastaxis of the QWP. In theory, the scattered LP light would convert to CP light and be equally divided by the PBSC causing the Raman LP light to cancel after subtraction (again after reconciling the different efficiencies of both channels). Considering the presence of $\mathrm{CP}$ in the excitation beam and optical rotation of the sample chamber, which is minute but sufficient to overwhelm the true ROA signal, such an alignment was not achievable. ${ }^{25}$

In the commercially available system ${ }^{6,12}$, this problem is avoided by total depolarization of the excitation beam using a half-wave plate (HWP) spinning at high rpm, as mentioned in Chapter 1, to achieve a time-averaged polarization state of zero. Moreover, an additional HWP is inserted in the excitation beam path for half of the exposure time to convert any RCP to LCP light and vice versa to cancel out any artifacts they would contribute. These highly customized optical mounts and specialized electronic controllers were unavailable and our efforts to construct our own, by consulting our local machine shop, were unsuccessful. Our attention then turned to ICP-ROA (Figure 4.2) and DCPI-ROA (Figure 4.3A). 


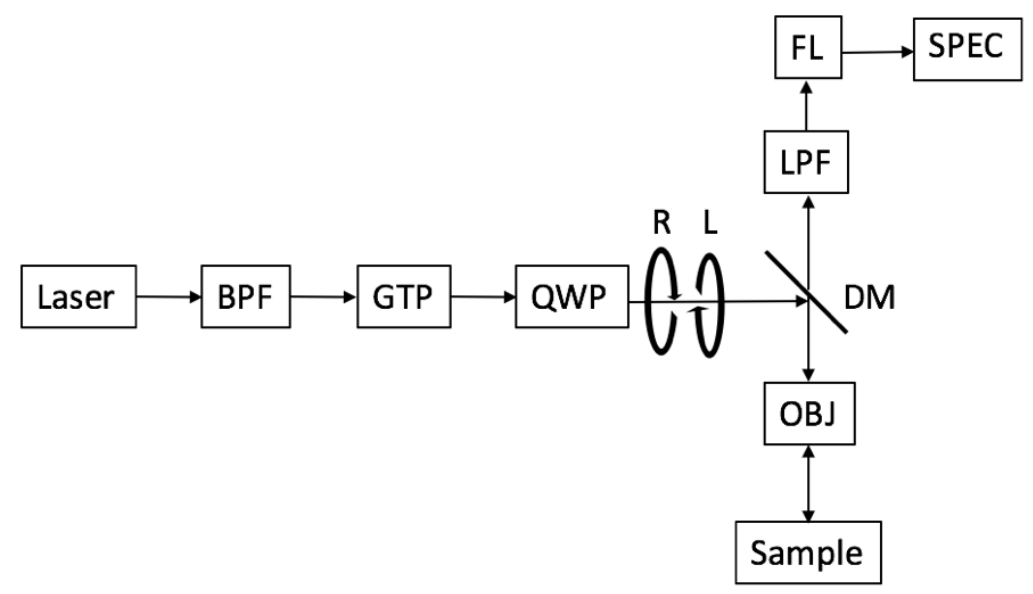

Figure 4.2 ICP-ROA optical diagram. Band-pass filter (BPF), Glan-Taylor polarizer (GTP), quarter-wave plate (HWP), objective lens (OBJ), dichroic mirror (DM), long-pass filter (LPF), fiber launch (FL), and spectrograph (SPEC)

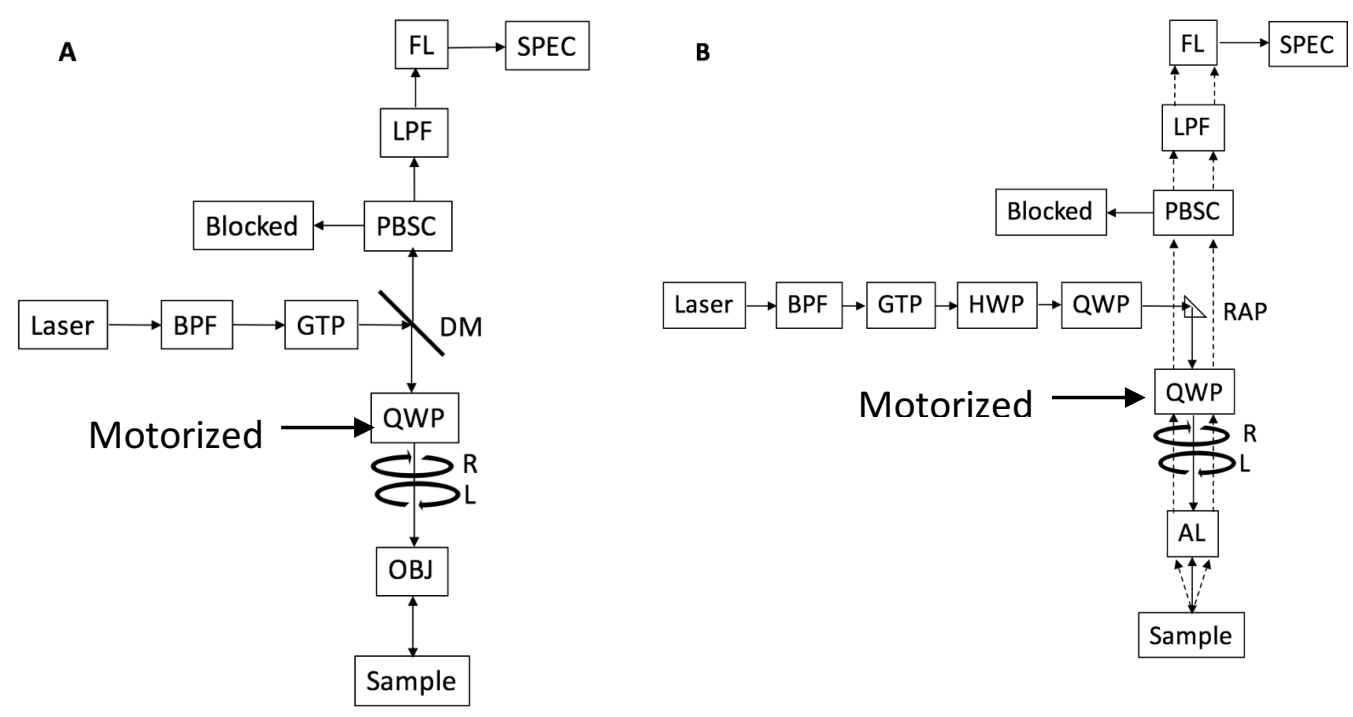

Figure 4.3 $\mathrm{DCP}_{\mathrm{I}}-\mathrm{ROA}$ optical diagram (A) and final working $\mathrm{DCP}_{\mathrm{I}}-\mathrm{ROA}$ optical diagram (B). Band-pass filter (BPF), Glan-Taylor polarizer (GTP), quarter-wave plate (QWP), half-wave plate (HWP), objective lens (OBJ), aspheric lens (AL), right-angle prism (RAP), dichroic mirror (DM), polarizing beam splitter cube (PBSC), long-pass filter (LPF), fiber launch (FL), and spectrograph (SPEC) 


\subsubsection{ICP-ROA}

At this stage, a commonly used standard of ROA, (+)- $\alpha$-pinene and its enantiomer (-)- $\alpha$-pinene, became available. ICP-ROA was quickly dismissed after numerous failed attempts at generating the ROA of $\alpha$-pinene, which were largely attributed to the poor quality of circularly polarized excitation light. Circularly polarized excitation light could not be generated such that the magnitude of the orthogonal EM components of the elliptical beam deviated by $1 \%$ or less. The least deviation achieved was around $10 \%$ most likely due to the fact the QWP was achromatic and not centered at $785 \mathrm{~nm}$. Currently, no QWP centered at $785 \mathrm{~nm}$ commercially exists as an off-the-shelf item.

\subsubsection{DCPI-ROA}

Attention was then turned to $\mathrm{DCP}_{\mathrm{I}}-\mathrm{ROA}$ as it was becoming increasingly clear that spectral artifacts were the major cause of problems. This configuration boasts unique advantages among all others as having the least susceptibility to artifacts, minimum parent Raman intensity, and reduced Rayleigh background intensity. $^{6,16,25}$ Initial attempts were also met with failure until it was determined that various components were causing changes to the polarization of both the exciting beam and scattered radiation. Firstly, the dichroic mirror (DM) was replaced by a right-angle prism after determining that such mirrors have polarization and wavelength dependent efficiencies due to its dielectric coating. In other words, passing through the DM caused all circularly polarized scattered radiation to turn elliptical and created bias in our measurements. Furthermore, the objective lens used also had polarization altering properties as it had a phase contrasting feature. In fact, any optic with any coating such as anti-reflective (AR) coatings was found to introduce contaminating polarization states reaching the sample. Although minute, the presence of these anomalies 
is sufficient to create significant spectral artifacts after long exposures using high power levels making it impossible to discern any delicate ROA features. Despite these changes, the incident beam was still not circularly polarized given the excitation beam polarization was altered by a right-angle prism due to the nature of total internal reflection. ${ }^{149}$ To compensate, a previously reported method was employed that involved the addition of a QWP in the excitation beam that could be micro-adjusted to compensate for the unwanted polarization changes throughout the setup. ${ }^{25}$ After laborious manual adjustments with trial and error, a fairly decent ROA spectra of $(+)-\alpha$-pinene was produced, shown in Figure 4.4, that was in close agreement with previous reports, one of which is shown in Figure 4.5 by the curve labeled ' $\mathrm{DCP}_{\mathrm{I}}$. ${ }^{6}$, 16, 150, 151 Incident light, emitted by laser of $785 \mathrm{~nm}$ (Toptica XTRA II), with about $300 \mathrm{~mW}$ reached the sample with an exposure time of 4 hours per polarization hand. Spectra was recorded by a spectrometer (Shamrock 303, Andor Technology, Belfast, UK) connected to a super cooled CCD optimized for NIR detection (DU920P-BRDD, Andor Technology, Belfast, UK). Cosmic ray rejection was enabled. See Figure 4.3B for an optical diagram of the finalized setup.

An analysis of the CID spectrum, shown in Figure 4.4, confirms the authenticity of all the ROA peaks since the vast majority were on the order of $10^{-3}$ of the parent Raman spectrum. However, a few exceptions with values that were greater than $10^{-2}$ orders of magnitude of the parent Raman spectrum were found between 1000 and $1600 \mathrm{~cm}^{-1}$. Clearly, some ROA values were not the result of the sample's chirality, and most likely caused by stray birefringence and other sources of spectral artifacts that remained. The overall drift of the CID spectrum indicated further fine tuned alignment was still required. Locations for some selected and valid ROA peaks are presented in Table 4.1. 
Table 4.1 CID values of selected ROA peaks

\begin{tabular}{cc}
\hline Raman Shift $\left(\mathrm{cm}^{-1}\right)$ & CID \\
\hline 605.8 & $3.3 \times 10^{-3}$ \\
658.6 & $7.9 \times 10^{-3}$ \\
922.2 & $6.3 \times 10^{-3}$ \\
950.7 & $5.7 \times 10^{-3}$ \\
1435 & $7.7 \times 10^{-3}$
\end{tabular}

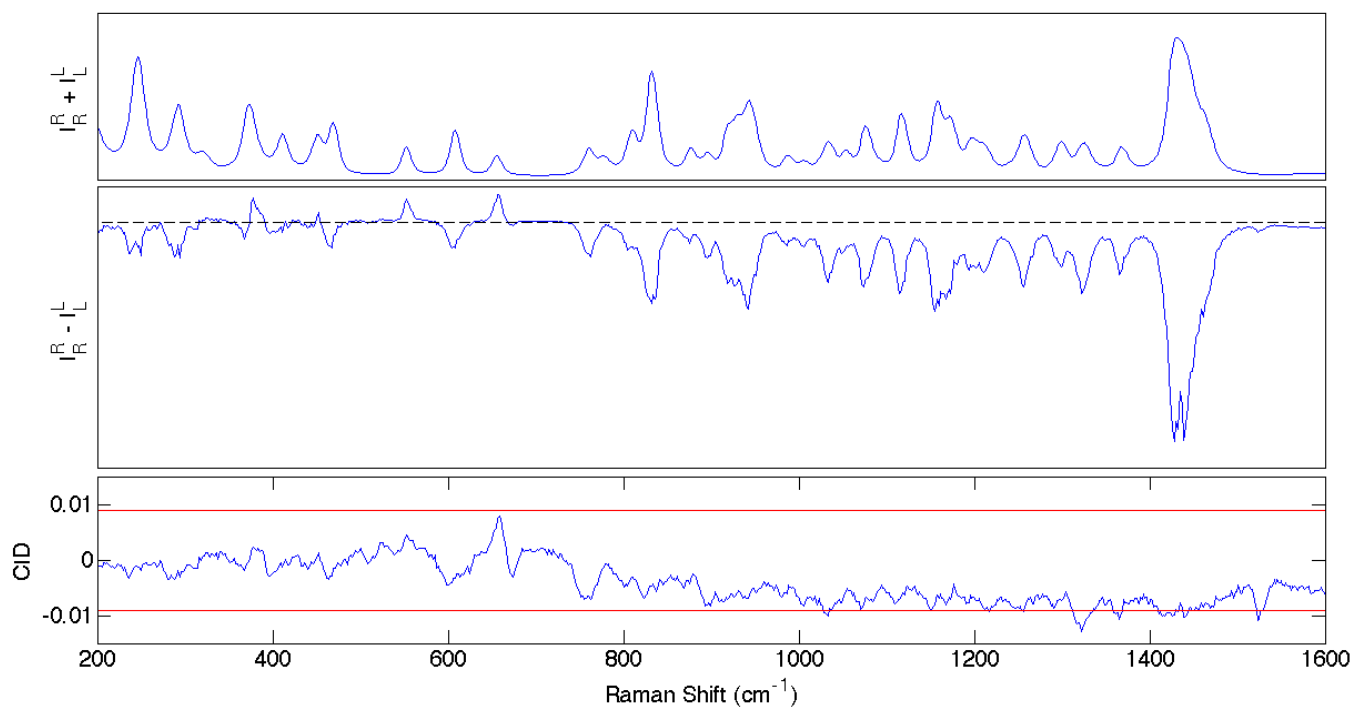

Figure 4.4 From top to bottom: the parent Raman, ROA (zero baseline indicated by dashed black line), and CID spectrum of (+)- $\alpha$-pinene. Values of $9 \times 10^{-3}$ and $-9 \times 10^{-3}$ are shown in red in the CID spectrum to highlight regions that were on the order of $10^{-3}$ to $10^{-5}$ of the parent Raman spectrum. 


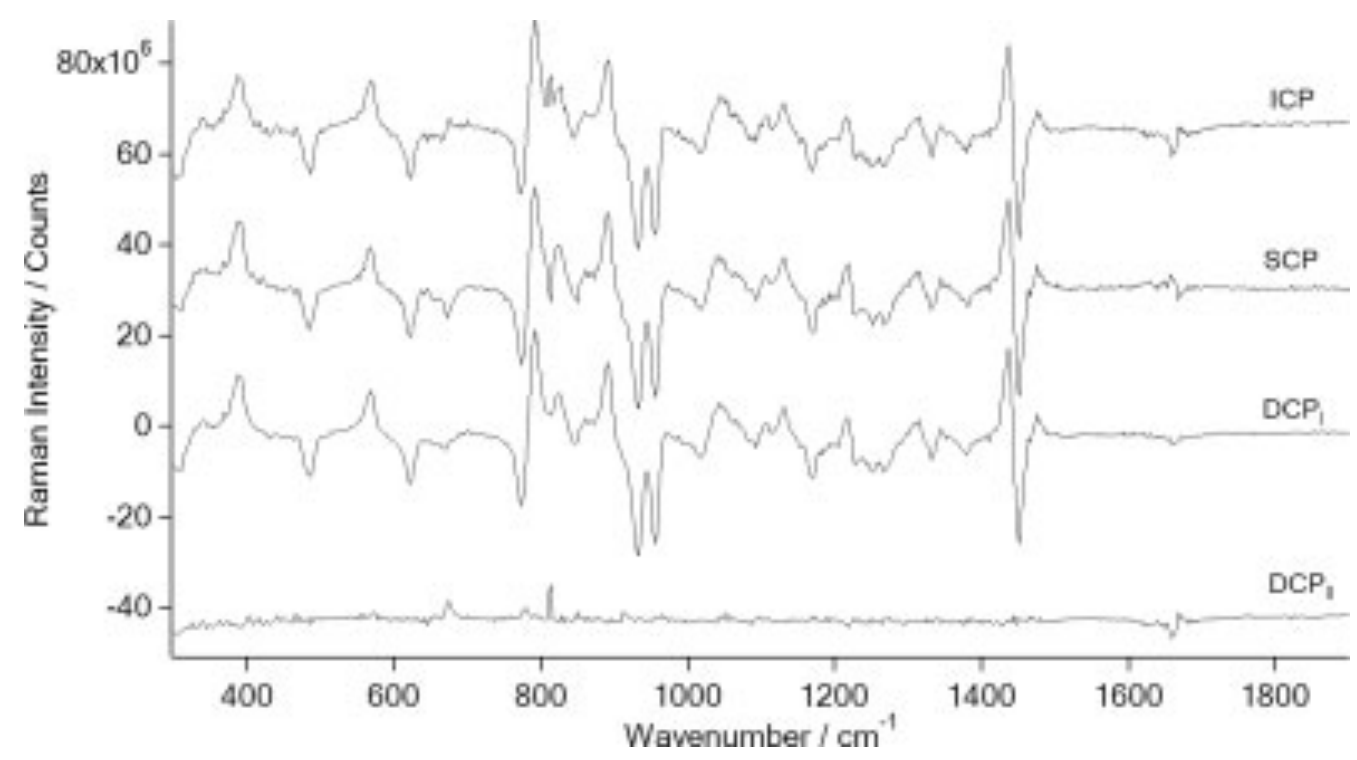

Figure 4.5 Measured ROA of (+)- $\alpha$-pinene in all four forms of ROA detection geometries. Adapted from "Simultaneous acquisition of all four forms of circular polarization Raman optical activity: results for $\alpha$-pinene and lysozyme," by H. Li and L. Nafie, 2012, Journal of Raman Spectroscopy, 43, p. 89-94. Copyright 2011 by John Wiley \& Sons, Ltd. Adapted with permission. 


\subsection{SERS and SEROA}

Attempts to obtain SEROA spectra of cytochrome c using silver nanoparticle and silver nanostars (AgNS) were ongoing during the construction and alignment stages of the SCP and ICP configurations. Needless to say, the SEROA results were all erroneous, confirmed by CID values in great excess of $10^{-3}$ and no similarity with published spectra. However, the parent SERS spectra were easily obtainable and provided valuable information about the performance of nanostars and the benefits of shorter excitation wavelengths.

\subsubsection{Silver Nanostar Synthesis}

Silver nanostars (AgNS) were explored to reduce SEROA exposure times to less than 30 minutes as have been previously reported with standard spherical AgNPs. ${ }^{140,143}$ Garcie-Leis et. al. ${ }^{44}$ purported that AgNS can still generate extremely strong SERS signals without aggregation since they exhibit plasmon resonance in the near-IR due to their size and irregular morphology.

All synthesis reaction flasks and accompanying glassware were soaked in aqua

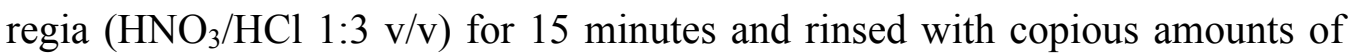
Milli Q water. Nanostars were then synthesized by combining hydroxylamine $\left(500 \mu \mathrm{L}, 6 \times 10^{-2} \mathrm{M}\right)$ and $\mathrm{NaOH}(500 \mu \mathrm{L}, 50 \mathrm{mM})$ in a round bottom flask. $\mathrm{AgNO}_{3}(9 \mathrm{~mL}, 1 \mathrm{mM})$ was added in one step followed by the addition of trisodium citrate $(100 \mu \mathrm{L}, 1 \% \mathrm{w} / \mathrm{v})$. Stirring was continued for 48 hours to allow nanostars to fully form. Although the resulting nanostars were a success, the resulting $10 \mathrm{~mL}$ of AgNS colloid was of insufficient quantity for conducting SERS measurements on a large array of protein concentrations. The volume 
was then scaled up to $700 \mathrm{~mL}$, but did not initially result in nanostar formation. After numerous attempts, it was determined that gradual addition, accomplished by controlled pouring, of a mixed hydroxylamine and $\mathrm{NaOH}$ solution to a stirring solution of $\mathrm{AgNO}_{3}$ (followed by addition of trisodium citrate) lead to the formation of crudely shaped nanostars, which were then explored for use in SERS. Both TEM and UV-VIS absorbance spectra are shown in Figure 4.7. At first glance, the original synthesis method produced relatively smooth surfaces that agree with Garcia-Leis et. al. ${ }^{44}$ Interestingly, the scaled up batch had a bumpy surface that suggests the existence of multiple nucleation sites. This strange and unexpected topography may have been caused by an overly rapid reaction rate caused by fast addition of the reducing agent solution. Despite this, the nanoparticles still resembled nanostars and exhibited a characteristic UV-VIS spectrum. Future implementation of a fluidic delivery system with a controllable flow-rate would be highly desirable to improve the reproducibility of this method.

\subsubsection{Spherical AgNP Synthesis}

$\mathrm{NaOH}(90 \mathrm{~mL}, 3.3 \mathrm{mM})$ was diluted 100 times with Milli Q water in a round bottom flask. Under rapid stirring, hydroxylamine hydrochloride (10.4 mg) was added and left to stir until fully dissolved. Finally, $\mathrm{AgNO}_{3}(10 \mathrm{~mL}, 10 \mathrm{mM})$ was added in one step and left to stir for 15 minutes. The resulting colloid displayed a plasmon resonance at around $410 \mathrm{~nm}$ indicating an average diameter of around $30 \mathrm{~nm}$. 


\subsubsection{SERS and SEROA of Cytochrome C with AgNS}

Cytochrome c (product number: C2037) from bovine heart was purchased from Sigma-Aldrich. Briefly, AgNS colloid $(180 \mu \mathrm{L})$ was mixed with cytochrome c $(10 \mu \mathrm{L}, 1 \mathrm{mg} / \mathrm{mL})$ followed by addition of $\mathrm{Na}_{2} \mathrm{SO}_{4}(20 \mu \mathrm{L}, 1 \mathrm{M})$. The colloid immediately darkened upon mixing with the protein solution, indicating aggregation, and darkened further after addition of salt. A $10 \mu \mathrm{L}$ drop of this mixture added to a flat aluminum substrate for Raman analysis. The sample was irradiated by a laser operating at $785 \mathrm{~nm}$ light (Toptica XTRA II) at about $80 \mathrm{~mW}$ with an exposure time of 30 seconds. Spectra was recorded by a spectrometer (Shamrock 303, Andor Technology, Belfast, UK) connected to a super cooled CCD optimized for NIR detection (DU920P-BR-DD, Andor Technology, Belfast, UK). Although not shown in the diagram, the optical setup was equipped such that it could be switched to act as a microscope for targeting aggregates. This addition was designed as a removable auxiliary to avoid affecting Raman experiments.

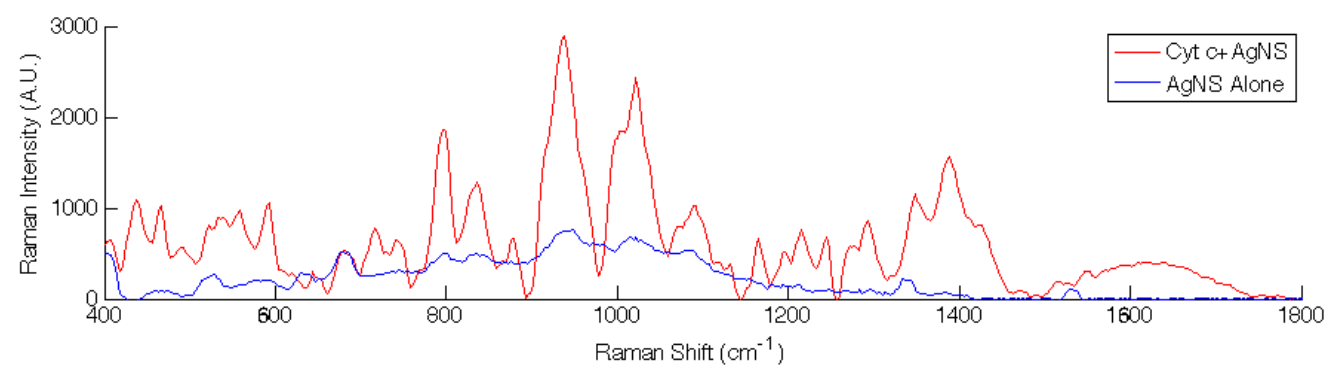

Figure 4.6 Raman spectrum of cytochrome c enhanced by AgNS and of AgNS alone after aggregation 
Spectra, one of which shown in Figure 4.6, revealed an unprecedented wealth of peaks that mostly conflicted with published results by Johannessen et al. ${ }^{143}$ reproduced in Figure 4.10. This is likely the result of nanostars' vastly irregular morphology, relative to spheres, that enabled the enhancement within protein areas that are otherwise inaccessible by spherical nanoparticles. This morphology may also have affected the adsorption characteristics of proteins causing shifts in peak location. Spectra of aggregated nanoparticles sans protein showed there was contribution by the AgNS in the SERS spectrum possibly due to the presence of citrate or hydroxylamine as a result of not centrifuging the AgNS to remove excess hydroxylamine. Although the intensity of bands was impressive, it was most prudent to replicate the results by Johannessen et. al. ${ }^{143}$ by following their procedure using spherical nanoparticles for validating the setup's preparedness. 

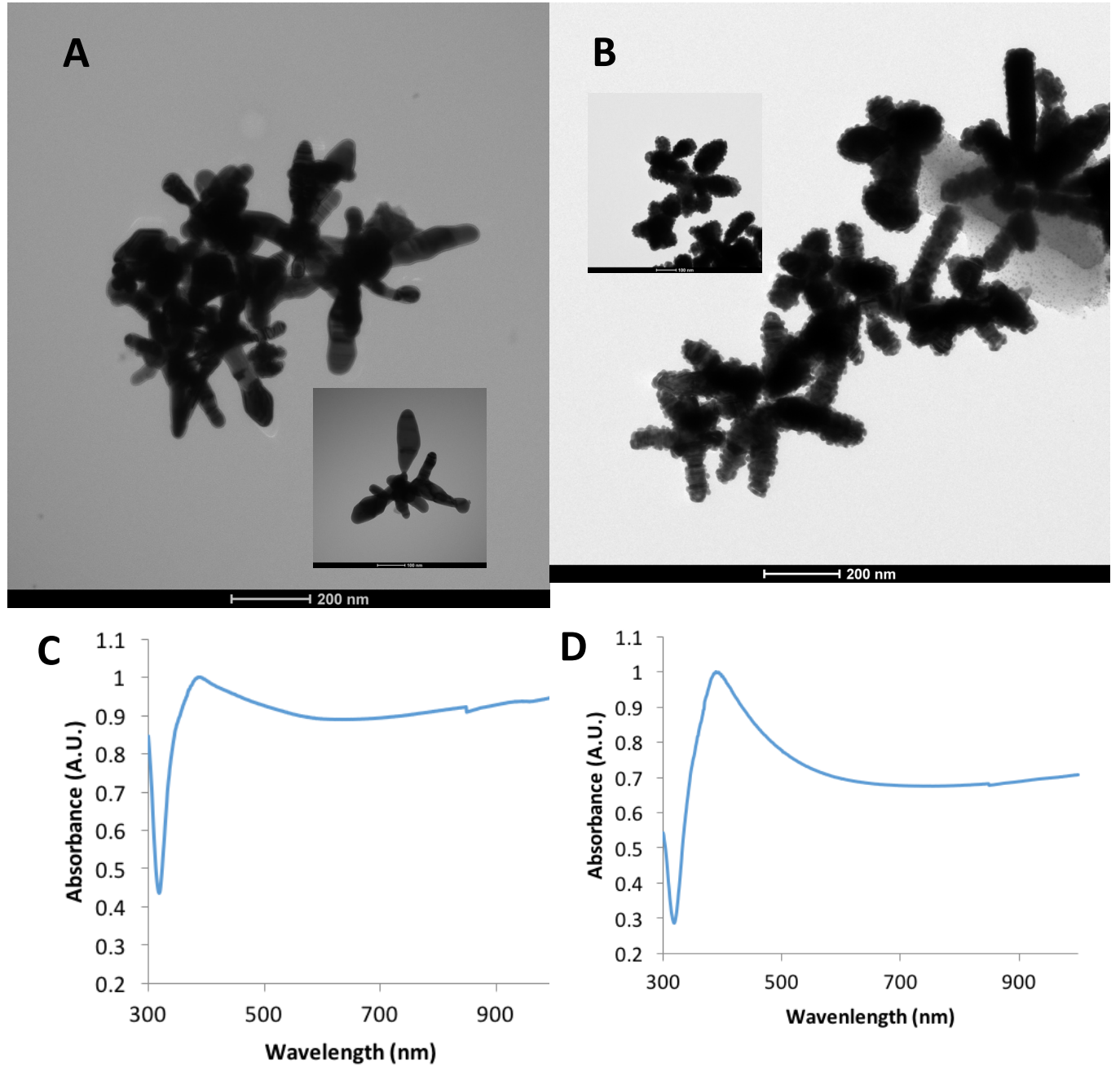

Figure 4.7 TEM micrograph of (A) small scale synthesis (inlay scale: $100 \mathrm{~nm}$ ) and (B) large scale synthesis of silver nanostars (inlay scale: $100 \mathrm{~nm}$ ). The respective UV-VIS spectra of both small (C) and large (D) scale batches are also presented.

\subsubsection{SERS and SEROA of Cytochrome C with Spherical AgNPs}

Protein and silver colloid were combined in the same way as done with AgNS.

In this instance, both $785 \mathrm{~nm}$ and $633 \mathrm{~nm}$ excitation radiation were used.

Spectra measured using $785 \mathrm{~nm}$ poorly matched that reported by Johannessen et al. ${ }^{143}$ (Figure 4.10) due to their use of $532 \mathrm{~nm}$ excitation light. However, after 
changing to $633 \mathrm{~nm}$, agreement was significantly improved owing to the activation of resonance as seen in Figure 4.8. The $\mathrm{x}$-axis was reversed to facilitate comparison with the published data. 


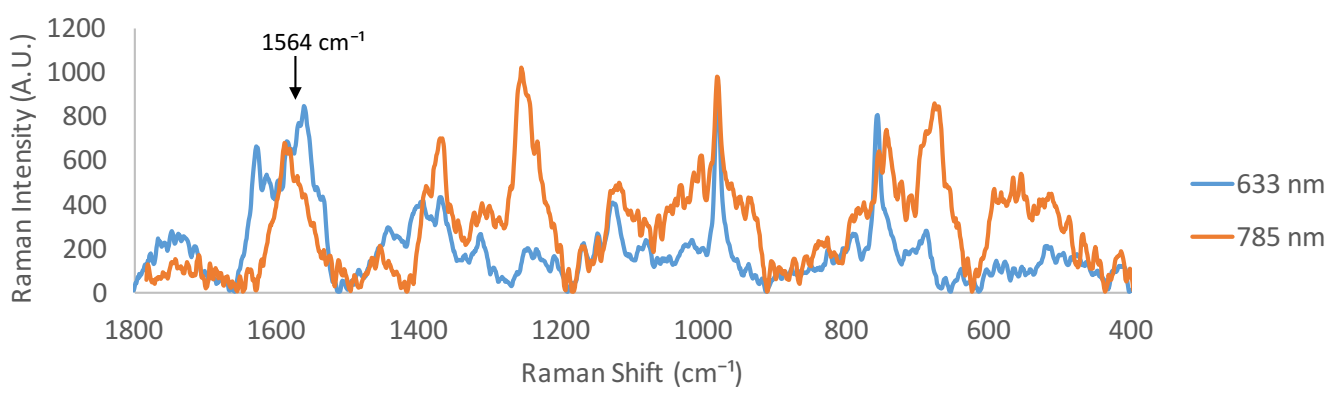

Figure 4.8 Cytochrome $\mathrm{c}$ and AgNPs SERS spectrum using 633 and $785 \mathrm{~nm}$ excitation

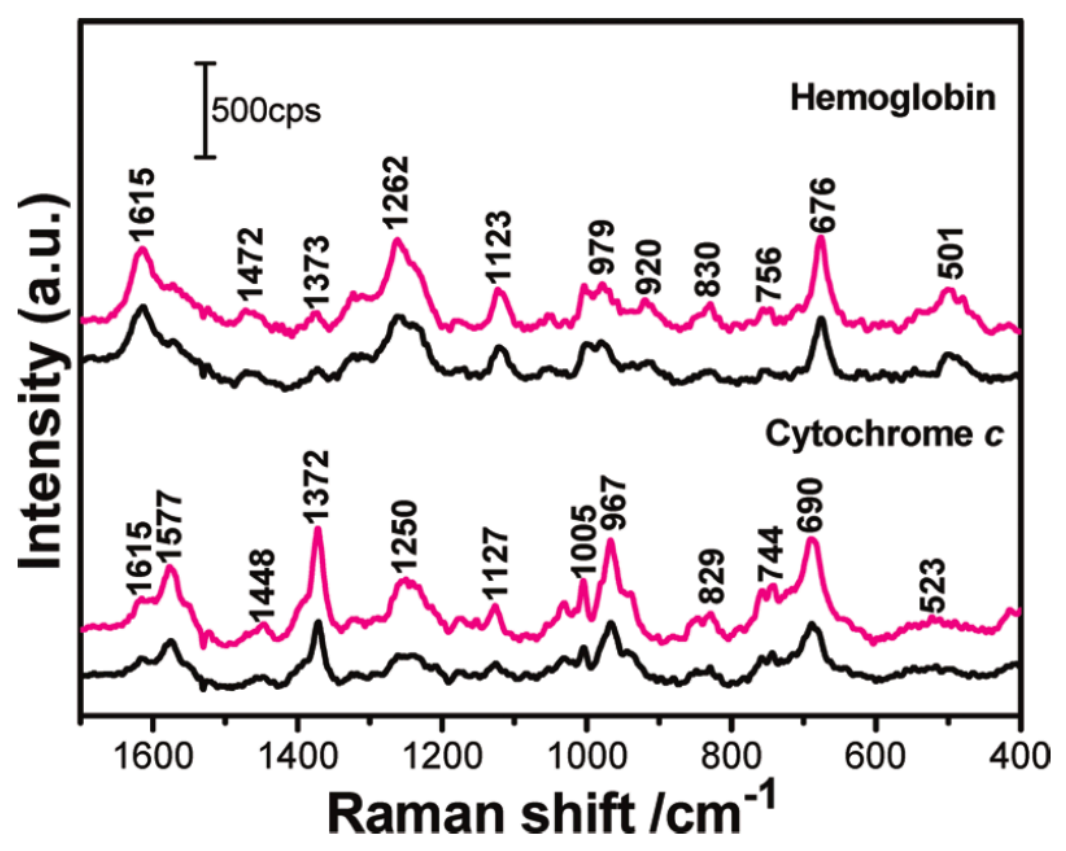

Figure 4.9 SERS spectra of hemoglobin and cytochrome. Adapted from "Label-free highly sensitive detection of proteins in aqueous solutions using surface-enhanced Raman scattering," by X. Han, G. Huang, and B. Zhao, 2009, Analytical Chemistry, 81, p. 3329-3333. Copyright 2009 by the American Chemical Society. Adapted with permission. 


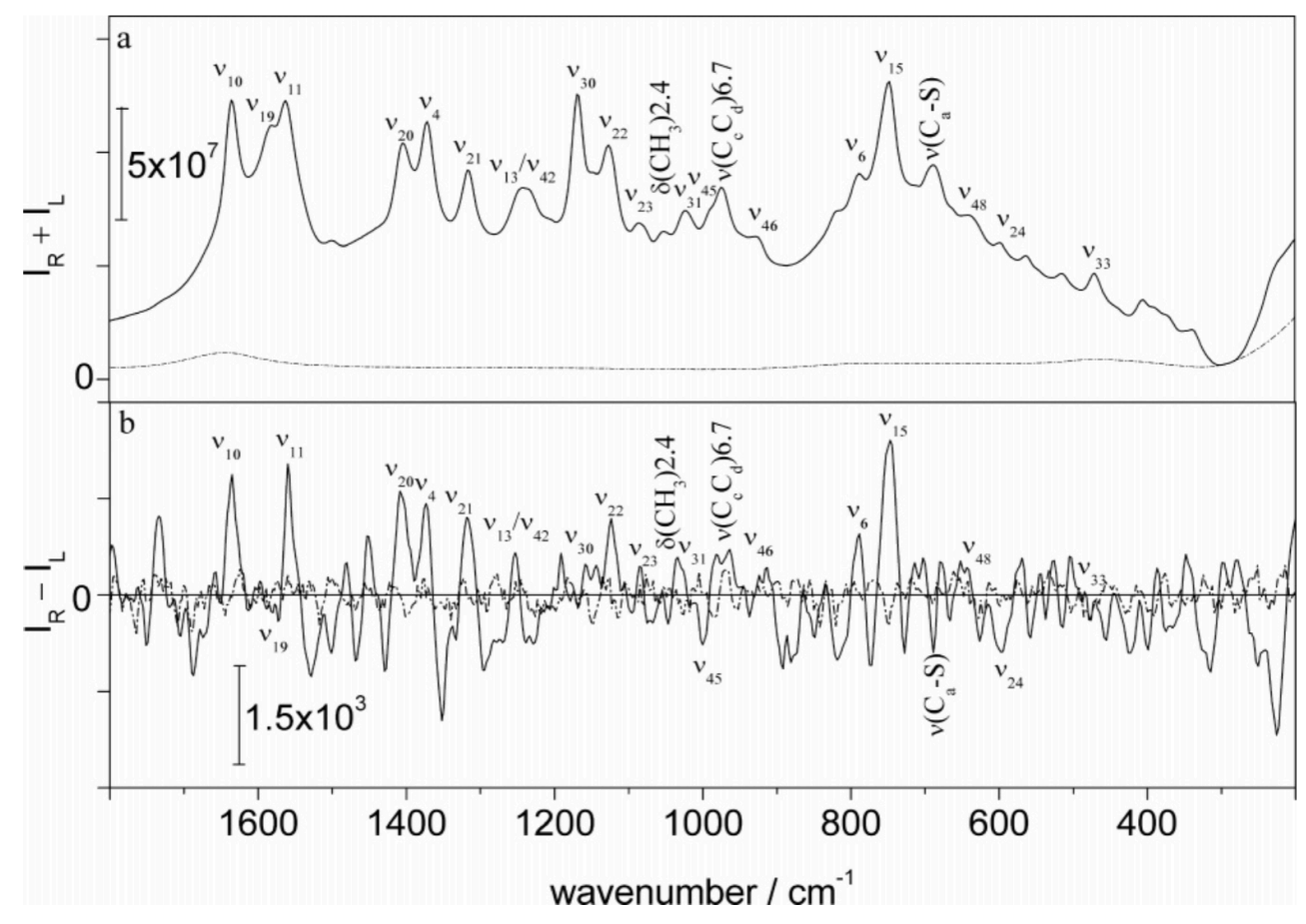

Figure 4.10 SERRS (a) and SERROA (b) spectra of cytochrome c in aqueous/colloidal solution. Control spectra of colloid and water mixtures are included in both figures (dotted lines). Adapted from "Resonance Raman Optical Activity and Surface Enhanced Resonance Raman Optical Activity Analysis of Cytochrome c," by C. Johannessen, P. White, and S. Abdali, 2007, Journal of Physical Chemistry, 111, p7771-7776. Copyright 2007 by the American Chemical Society. Adapted with permission.

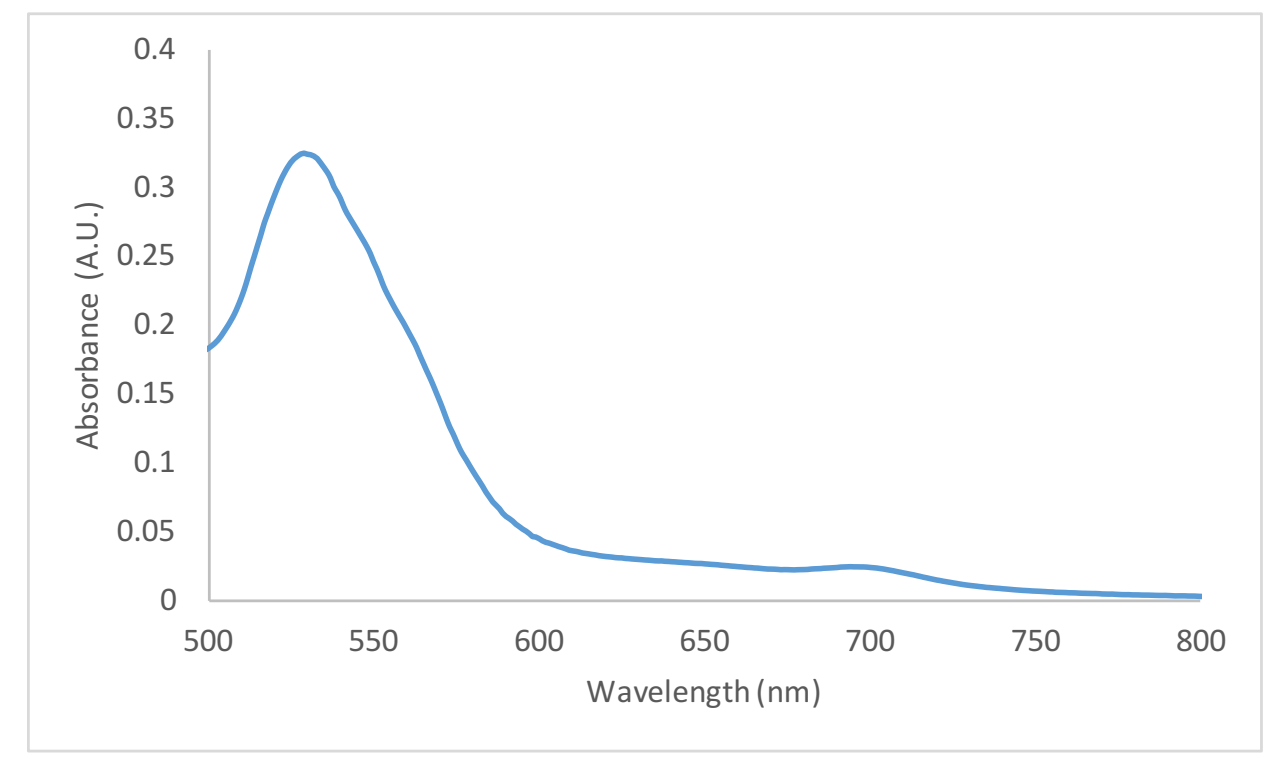

Figure 4.11 UV-VIS absorbance of cytochrome $\mathrm{c}$ in solution 
Cytochrome c, like all proteins containing porphyrin, displays strong absorbance bands in the visible region ranging approximately from 400 to 750 $\mathrm{nm}$. Cytochrome $\mathrm{c}$ has a peak absorbance at around $532 \mathrm{~nm}$ as shown in Figure 4.11. Excitation at this wavelength triggers an electronic transition which gives rise to the resonance Raman (RR) effect and enhances Raman scattering by several orders of magnitude. When combined with SERS, the effect is called Surface Enhanced Resonant Raman scattering (SERRS). Therefore, the study by Johannessen et. al. ${ }^{143}$ benefited from the enormous enhancement provided jointly by these two effects meaning they actually reported the SERRS and SERROA spectra of cytochrome c. Excitation using $633 \mathrm{~nm}$ also evoked resonance since the protein shows absorbance at this location. As result, it is not surprising, in addition to the contribution of the $1 / \lambda^{4}$ dependence, that close agreement was found. Unfortunately, no resonance is active at $785 \mathrm{~nm}$ resulting in a degraded spectrum where finer details were overlapped by broader peaks as is the case, for example, at around $1564 \mathrm{~cm}^{-1}$. Fortunately, the results using $785 \mathrm{~nm}$ had excellent agreement with the SERS spectra reported by Han et. al. ${ }^{45}$, reproduced in Figure 4.9, whom used the same excitation wavelength on cytochrome c and citrate capped AgNPs.

\subsection{Discussion}

Our results have verified claims that $532 \mathrm{~nm}$ is an ideal choice for laser excitation. In our case, $633 \mathrm{~nm}$ would be preferable to maximize the amount of information contained within the spectra. Unfortunately, a minimum of 300 $\mathrm{mW}$ is required for alignment and calibration using the pinene standards. Sadly, the only available $633 \mathrm{~nm}$ laser can achieve a maximum output of $21 \mathrm{~mW}$ from 
its front end. After passing through numerous optics within the setup, this power was reduced to around $10 \mathrm{~mW}$, which was not sufficient for even measuring SERROA. Consequently, $785 \mathrm{~nm}$ was the only remaining option at our disposal where about $300 \mathrm{~mW}$ reaching the sample was achieved in freespace mode. ROA instruments have been constructed using $785 \mathrm{~nm}$ and other near-IR wavelengths. However, they do not have the RR advantage. Larger AgNPs were explored in order to achieve a post-aggregation resonance band around $800 \mathrm{~nm}$ nearer to $785 \mathrm{~nm}$ to compensate, but this made no noticeable improvement in sensitivity. Briefly, these larger AgNPs were synthesized by slow and controlled addition of glucose to a silver nitrate solution at basic $\mathrm{pH}$; the resulting LSPR band was located at $427 \mathrm{~nm}$ indicating an average diameter of $52 \mathrm{~nm}$.

Given the absence of porphyrin that limits resonance to the deep UV region, performing SERROA measurements in the deep UV range is an attractive idea given the strong absorption maxima between 275 and $280 \mathrm{~nm}$ exhibited by the aromatic amino acids tryptophan and tyrosine. ${ }^{152}$ But increased chance of photodegradation by UV light may present too great an obstacle. Moreover, finding plasmonic materials that support SERS in the UV remains a major challenge where enhancement factors of only up to 100 have been reported. ${ }^{153}$ Despite absence of resonance at $785 \mathrm{~nm}$, SEROA spectra of such proteins may still be within reach when considering the use of nanostars or other SERS substrates with morphologies optimized for proteins in solution. Although the resulting spectra cannot be directly compared to previous reports, they are verifiable by calculation of the CID for each suspected SEROA peak. 
Direct contact with nanoparticles can cause proteins to bind strongly to the surface and vastly alter their structure. This is a concern worth noting since the importance of preserving native structure goes without saying. The high similarity between SEROA and ROA of cytochrome c presented by Johannessen et al. ${ }^{143}$ strongly suggested that no significant structural changes occurred. This is most likely due to a monolayer of chlorine anions ${ }^{154}$ adsorbed onto the surface of each nanoparticle, which is a consequence of the synthesis method used, namely the Leopold and Lend1 ${ }^{155}$ method where hydroxylamine hydrochloride is the reducing agent. Stabilizing surface ions that are more weakly bound to the surface, such as sulfate and citrate, are not ideal since target molecules can easily displace them. ${ }^{45,154}$ In one particular study, Xu et. al. ${ }^{36}$ demonstrated SERS detection of protein in their native structure by replacing citrate with strongly binding halide ions: iodine, bromine, and chlorine. Interestingly, Abdali et. al. ${ }^{140}$ has reported SEROA of myoglobin using citrate-capped AgNPs and showed high similarity between SEROA and ROA spectra. Although similar, it may be explained by the short exposure time used (5 minutes) in which a structural change was still in its nascent stages and therefore negligible. It may also be the case that myoglobin in particular cannot readily bind and subsequently displace the citrate anions given its relatively neutral charge at $\mathrm{pH} 7$. A discussion of future work is given in Chapter 6. 


\section{Chapter 5 Light and Temperature Sensitive Polymers for Modulated Surface Enhanced Raman Scattering}

\subsection{Introduction}

Pursuant to selective detection of proteins against a background of such molecules, a modulated signal approach was attempted. In essence, the distance between a target protein molecule and a surface plasmon is periodically lengthened and shortened by means of a stimuli responsive polymer sensitive to light or heat. By selecting a single protein using its associated antibody, its modulated SERS signal could be recognized against a background of other proteins that maintain a steady signal. This would enable the detection of single protein markers important in blood diagnosis and stem cell differentiation without the complex separation methods already mentioned. $^{156}$ A modulated signal, if of sufficiently high frequency to overcome spectral noise, also offers an added advantage of heightened sensitivity allowing access to the nano and, potentially, picomolar range despite a preponderant presence of other molecules that may otherwise mask a target protein's signal. Furthermore, the intrinsic Raman spectra after enhancement rather than a Raman reporter may yield useful information about protein conformation.

To that end, creation of a photoresponsive polymer incorporating azobenzene, a photosensitive molecule, was attempted. It was posited that azobenzene functionalized monomers incorporated into a linear polyacrylic acid chain would impart it with the ability to reversibly change in hydrophobicity in response to external light. Under visible light, the polymer coil becomes 
hydrophobic and agglomerates upon itself. Under UV light, the polymer regains its hydrophilicity and re-expands. Attachment of a target protein to one end of the chain while the other end is anchored to a plasmonic surface could, in principle, modulate the SERS of the attached protein in solution provided that the protein is brought close enough and that the polymer does not interfere with LSPRs. Put succinctly, SERS could be photonically switched on and off at will.

Another well known and studied polymer, poly(N-isopropylacrylamide) or polyNIPAM for short, was also explored for modulated SERS. This polymer becomes hydrophobic beginning at around $32^{\circ} \mathrm{C}$ which is known as the lower critical solution temperature (LCST) at which point it precipitates. At temperatures below the LCST, polyNIPAM is re-hydrated and returns to solution.

For simplicity, one end of the polymers was covalently attached to biotin for binding specifically to avidin. This was a prudent choice given that the biotinavidin interaction is the strongest known non-covalent bond making for convenient linkage. It also required straightforward chemistry to attach biotin to the end of the polymer chain by formation of an amide linkage.

This project was carried out in collaboration with the Institute of Chemical and Engineering Sciences (ICES) remotely located on Jurong Island. All synthesis reactions were performed at the ICES polymer lab supervised by Dr. He Tao. Dr. He, who was still an ICES member during my time there, was instrumental 
in establishing our collaboration with ICES. During my limited time there, I was fortunate to work with Dr. Alexander William Jackson who mentored me in methods of small molecule and polymer synthesis as well as essential lab techniques in organic chemistry. He also provided invaluable advice in guiding our direction of research.

In this work, we attempted to achieve modulated SERS with the said polymers attached to silver and gold spherical nanoparticles while in solution. Modulating the solubility state of the polymer would in turn modulate the colloid's state of aggregation. We also evaluated this scheme when nanoparticles were immobilized onto a glass substrate.

\subsection{Chemical Synthesis}

All polymer synthesis was performed using reversible addition-fragmentation chain transfer (RAFT) polymerization. This method was chosen because it can form polymers with narrow polydispersity ${ }^{157,158}$ and provided a convenient way to modify both ends of the polymer chain to suit our needs. All synthesized molecules were verified by NMR spectroscopy. Synthesis of monomers and small molecules was monitored using thin-layer chromatography (TLC) and purified by column chromatography. Polymers were purified by repeated precipitation in hexane. All chemicals were available at ICES while only 4hydroxyazobenzene and acryolyl acid were purchased from Sigma-Aldrich and Merck, respectively. In the following section describing synthesis procedures, each chemical component will be accompanied by a number in bold that corresponds to a reaction flowchart provided within the text. 


\subsubsection{Light Sensitive Polymer Synthesis}

\section{Azobenzene-Containing Monomer}

4-hydroxyazobenzene (1) and triethylamine were dissolved in tetrahydrofuran (THF) in a round bottom flask. After cooling to $0^{\circ} \mathrm{C}$, acryloyl acid (2) was added to a drop funnel containing a small volume of THF, after which the entire mixture was added drop-wise into the reaction chamber. The reaction was stirred for 1 hour. The resulting azobenzene-containing monomer (3) was purified by filtration followed by removal of the solvent using a rotary evaporator. Refer to Figure 5.1.

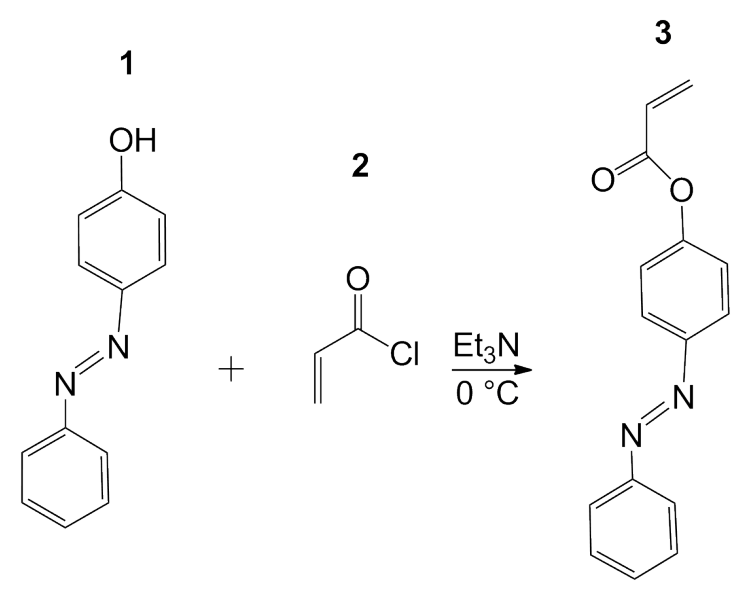

Figure 5.1 Azobenzene containing monomer synthesis

\section{Light Sensitive Copolymer}

The polymerization reaction was attempted twice in 1,4-dixoane and dimethylformamide (DMF).

2-(Dodecylthiocarbonothioylthio)-2-methylpropionic acid (DDMAT) (4), azobenzene-containing monomer (3), acrylic acid (9), and the initiator azobisisobutyronitrile (AIBN) were dissolved in either 1,4-dioxane or DMF within a Schlenk tube. Stabilizing agents present in the acrylic acid stock 
solution were removed by quickly passing through a small silica gel column. The azobenzene monomers were chosen to have a molar equivalence of $10 \mathrm{x}$ and arcylic acid of 90x relative to DDMAT to achieve a degree of polymerization (DP) of 100 . The mixture was degassed by the standard freezethaw method (using liquid nitrogen) and finally maintained in a nitrogen gas atmosphere. The reaction was stirred overnight at $70^{\circ} \mathrm{C}$ in an oil bath. After completion, the reaction was halted by freezing in liquid nitrogen. Polymers were purified by repeated precipitation in hexane and decanting. Filtering could not be done since the polymers, having a cumbersomely thick and oily form, became inseparable from filter paper. Refer to Figure 5.2 for a flowchart of synthesis.
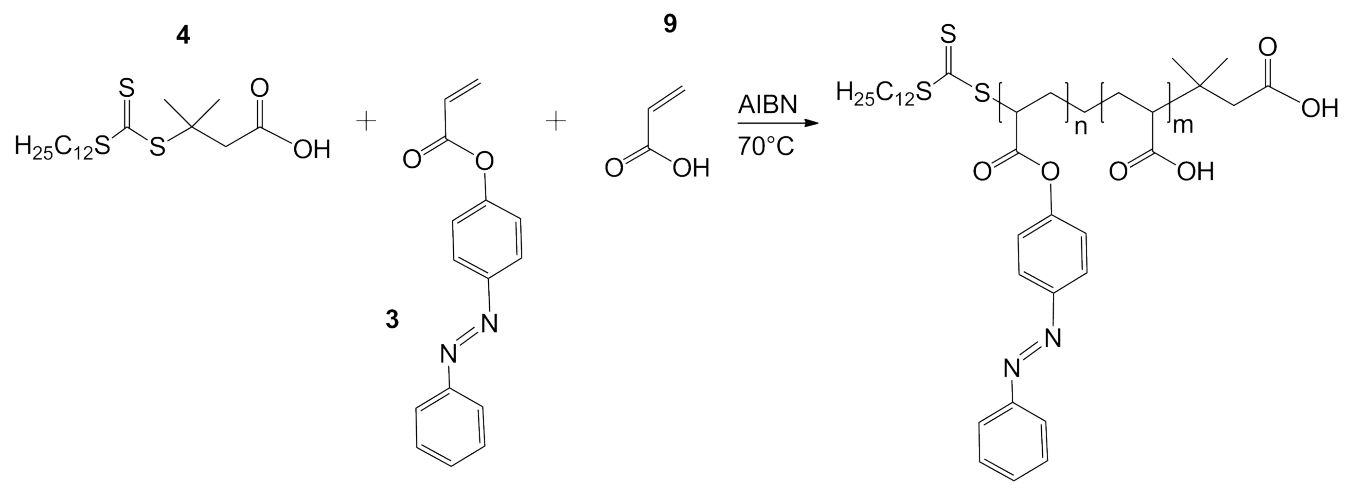

Figure 5.2 RAFT Synthesis of photoresponsive polymer

\subsubsection{Light Sensitive Polymer Results and Discussion}

All attempts at polymerization of the light sensitive polymer only achieved a DP of 30 despite trying 1,4-dixoane and DMF as solvents. The final DP was determined by NMR spectroscopy. An additional attempt where the acrylic acid was purified by distillation also resulted in the same stunted polymerization. Reasons leading to this failure are numerous and may include a non-ideal choice of solvent. It may be that the reactants had more affinity for 
the solvent than to themselves, which could have impeded the polymerization process. The azobenzene-containing monomers' collective effect on the entire polymer chain as it extended may have also caused the process to stop at $\mathrm{DP}=30$. Full control over DP was essential for determining the optimum length for modulated SERS.

Considering the vast number of variables involved in this problem, the insufficient time allotted to us at ICES, and the absence of a proof of concept with regard to modulating the SERS signal in this fashion, it was decided to abandon photoresponsive polymers. This conclusion was reached after much deliberation and by strong recommendation by our ICES collaborators who asserted the unworkability of this approach given the restraints of our situation. Temperature sensitive polymers became the next focus of our research since they have been widely studied and can be synthesized expediently.

\subsubsection{Temperature Sensitive Polymer}

\section{Biotinylation of RAFT Chain Transfer Agent}

DDMAT (4), N-boc-3-aminopropanol (5), N,N'-dicyclohexylcarbodiimide (DCC), and 4-dimethylaminopyridine (DMAP) were combined in chloroform and allowed to react for two days. The resulting product (6) was then deprotected by treatment with trifluoroacetic acid (TFA) in dichloromethane (DCM) to install an amine functional group onto the DDMAT molecule (7).

The biotin (10) carboxylic acid group was activated by N-hydroxysuccinimide (NHS) (8). Both biotin and NHS were dissolved in hot DMF. DCC was added 
and the reaction was allowed to proceed overnight. Crystalized byproducts were removed by filtration and the solvent removed by evaporation. (11) and (4) were dissolved in DMF and triethyamine was added. The reaction was left to run overnight followed by solvent removal and column chromatography. Refer to Figure 5.3 for a flowchart of synthesis.
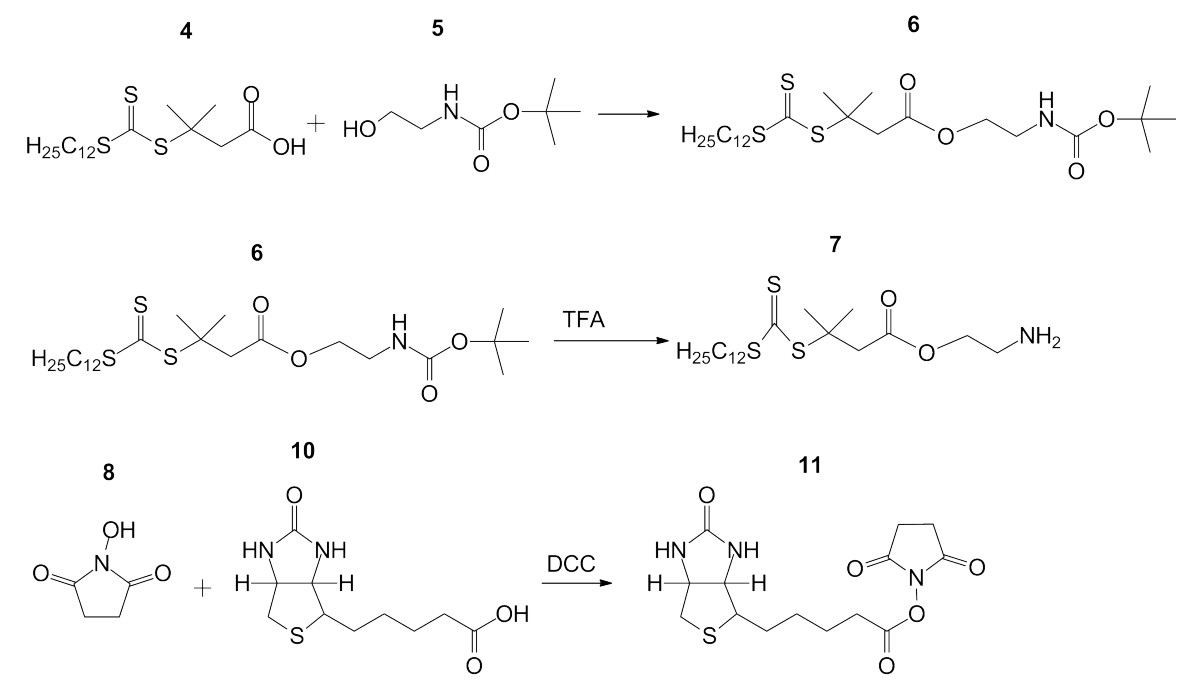

11

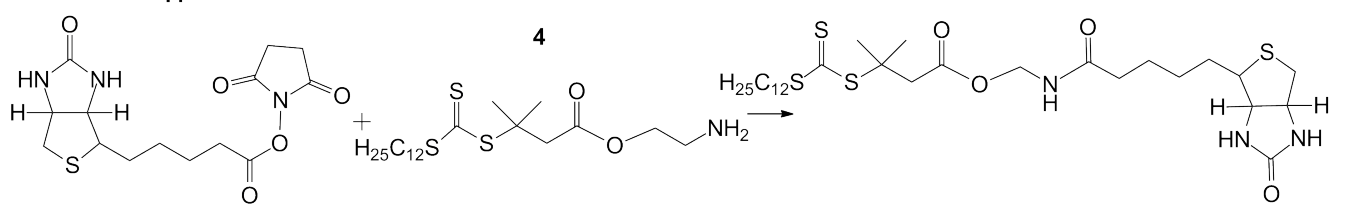

Figure 5.3 Biotinylation of RAFT chain transfer agent

\section{PolyNIPAM Synthesis}

Synthesis of polyNIPAM was performed using RAFT as was done with the light sensitive polymer. Briefly, biotinylated-DDMAT (12) was combined with $\mathrm{N}$-isopropylacrylamide (NIPAM) along with AIBN. NIPAM was added to a molar equivalence of 200x relative to biotinylated-DDMAT to target a DP of 200 and approximate molecular weight of $21 \mathrm{kDa}$. Refer to Figure 5.4 for a flowchart of synthesis. 


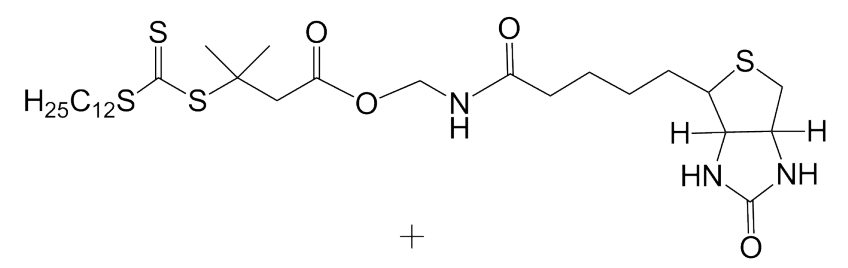

13

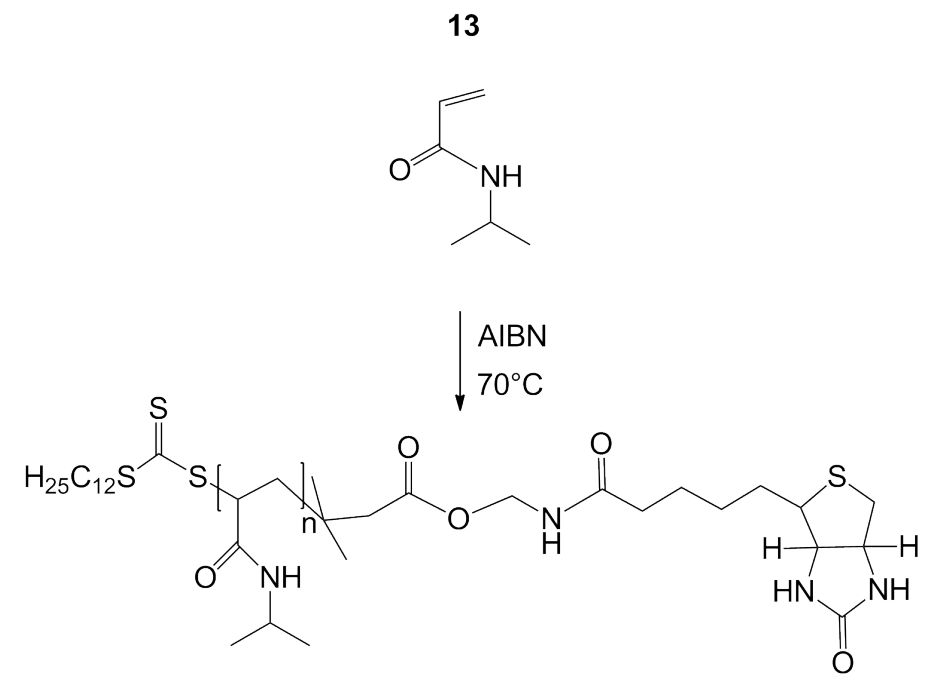

Figure 5.4 RAFT synthesis of polyNIPAM

A thiol was created on the leftmost end of the polymer by a cleavage reaction. Briefly, polyNIPAM-biotin was combined with 3-amino-1-propanol in THF at $60{ }^{\circ} \mathrm{C}$ and left stirring overnight. The vessel was first degassed and then maintained in a nitrogen atmosphere during the reaction. Refer to Figure 5.5 for the final result step.

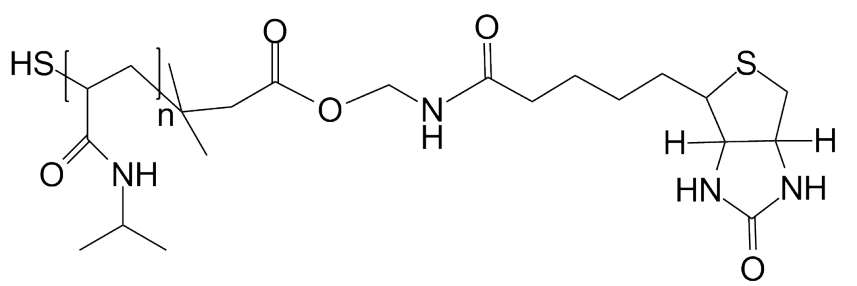

Figure 5.5 Thiolated and biotinylated polyNIPAM 


\subsubsection{AgNP Synthesis}

AgNPs were synthesized using the Lee and Meisel method. ${ }^{146}$ In this method, citrate serves as both the reducing and capping agent, which is a weakly bound anion easily displaced by incoming thiols. In a typical reaction, $\mathrm{AgNO}_{3}(17 \mathrm{mg})$ was dissolved in milliQ water $(100 \mathrm{~mL})$ and brought to a rapid boil under vigorous stirring. Trisodium citrate $(1 \% \mathrm{w} / \mathrm{v}, 2 \mathrm{~mL})$ was added gradually and the reaction was allowed to reflux for about 1 hour.

\subsubsection{AuNP Synthesis}

AuNPs of various sizes were synthesized using the seeded growth method reported by Bastus et. al. ${ }^{159}$ The entirety of the procedure will not be explicated here due to its lengthiness. Seed AuNPs were first synthesized in a citrate solution boiling at $100{ }^{\circ} \mathrm{C}$. Subsequently, the temperature was reduced to $90{ }^{\circ} \mathrm{C}$. Gold salt and additional sodium citrate was gradually added in discreet steps to create each size-generation (G0, G1, and so forth). All sizes synthesized are tabulated in Table 5.1, which includes the hydrodynamic diameter, polydispersity index (PDI), and position of the UV-VIS absorption peak. The concentration for each batch was estimated using the UV-VIS peak intensity value (provided it was below 1) and the corresponding extinction coefficient that is readily available on Sigma-Aldrich's website. The hydrodynamic diameter and PDI were determined using dynamic light scattering (DLS). Intensity data from DLS is presented in Figure 5.6. 
Table 5.1 DLS of size and PDI, and UV-VIS LSPR peak location of each size generation of AuNPs

\begin{tabular}{|c|c|c|c|}
\hline Designation & Size (nm) & PDI & UV-VIS LSPR (nm) \\
\hline Seed & 19.7 & 0.126 & 522 \\
\hline G0 & 31.8 & 0.069 & 523 \\
\hline G1 & 41.7 & 0.074 & 526 \\
\hline G2 & 48.2 & 0.081 & 529 \\
\hline G3 & 55.3 & 0.096 & 533 \\
\hline G4 & 65.8 & 0.111 & 538 \\
\hline
\end{tabular}

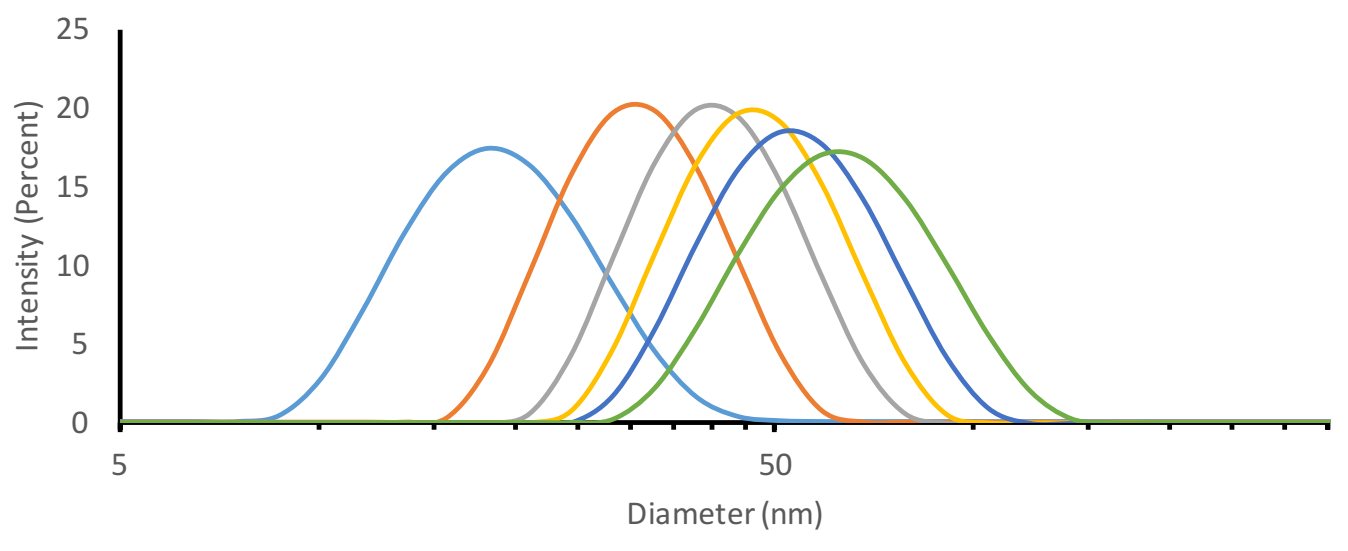

- Seed $-\mathrm{G} 0-\mathrm{G} 1-\mathrm{G} 2-\mathrm{G} 3-\mathrm{G} 4$

Figure 5.6 DLS intensity curves of each size generation of AuNPs 


\subsubsection{Conjugation of polyNIPAM with Nanoparticles}

Synthesized polyNIPAM was added in large excess directly to AuNPs ( $d \approx 55$ $\mathrm{nm})$ colloid and allowed to react for 48 hours while stirring. Excess polymer chains were removed by centrifugation twice (2000 g, $30 \mathrm{~min})$ and resuspended in four solutions: water, $\mathrm{KI}(100 \mathrm{mM}), \mathrm{NaCl}(50 \mathrm{mM})$, and PBS (1x, pH 7.5). See Figure 5.7.

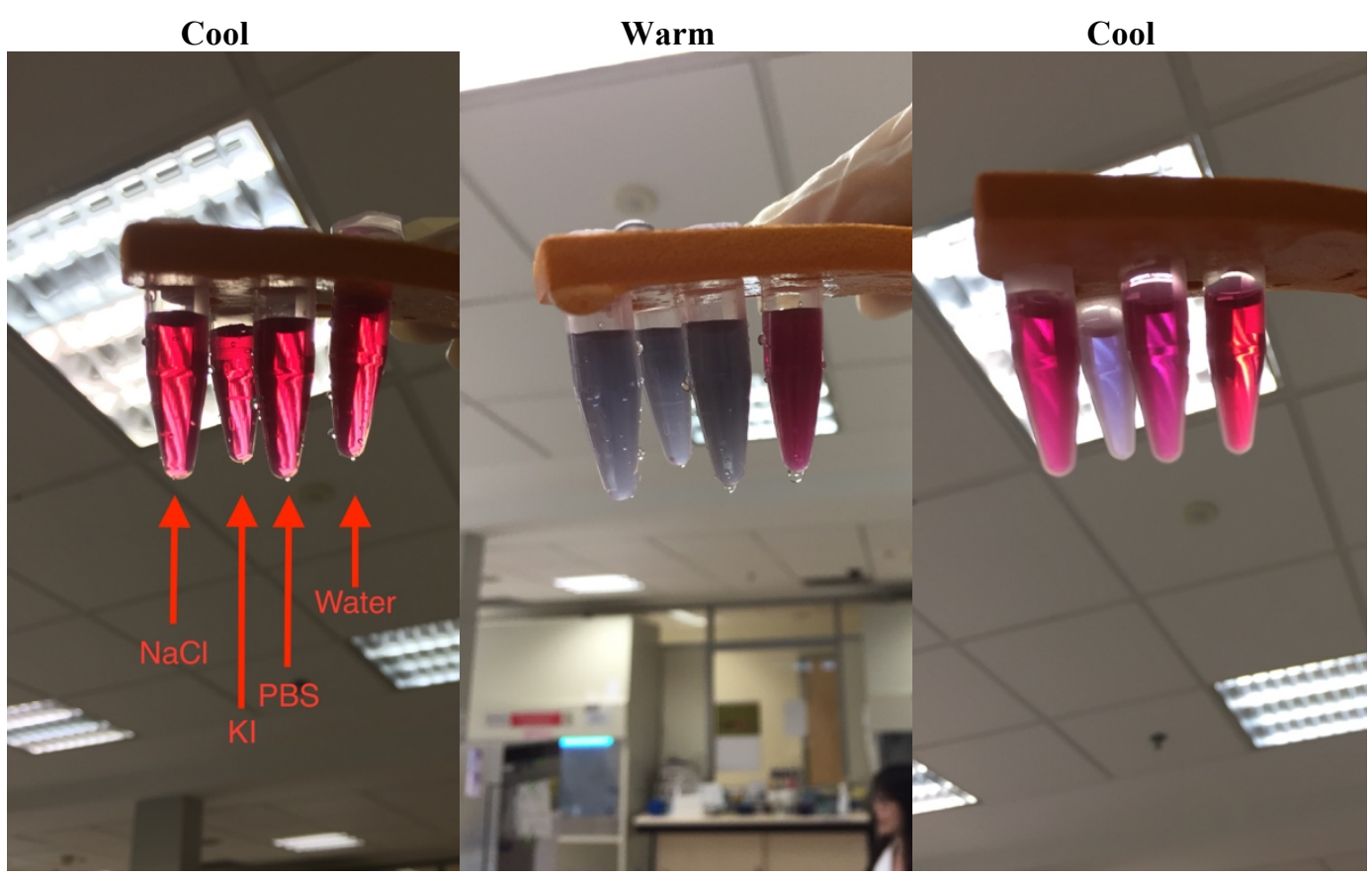

Figure 5.7 Reversible aggregation of polyNIPAM functionalized AuNPs

Upon heating to about $40{ }^{\circ} \mathrm{C}$ and cooling in an ice bath for 60 seconds, nearly all displayed a reversible color change from bright red (dispersed state) to dark purple (aggregated state). The colloid in water, however, displayed less aggregation (as shown by its color not having achieved a dark purple) indicating greater stability in a solution of low ionic strength. It was therefore not surprising when this colloid fully regained its original dispersed state below the LCST. In contrast, aggregation could not be reversed with KI perhaps due to its high concentration. By visual inspection, the colloids in $\mathrm{NaCl}$ and PBS 
did not appear to return fully to the original bright red hue upon cooling below the LCST, which indicated that some aggregates remained. Nevertheless, these results were consistent with previous reports in which aggregation only occurred in the presence of salt. ${ }^{160,161}$ The UV-VIS absorbance profile of the colloid in $\mathrm{NaCl}$ before heating (RT 1), after heating (W 1), and after cooling (RT 2) was consistent with observations as shown in Figure 5.8. Heating created a new plasmon peak at $642 \mathrm{~nm}$ that favored excitation with $633 \mathrm{~nm}$ to induce resonance. After returning to RT, the plasmon peak did not return fully to $539 \mathrm{~nm}$, in both position and intensity, and still showed slightly elevated absorbance after $550 \mathrm{~nm}$ and onward connoting the remnants of aggregates.

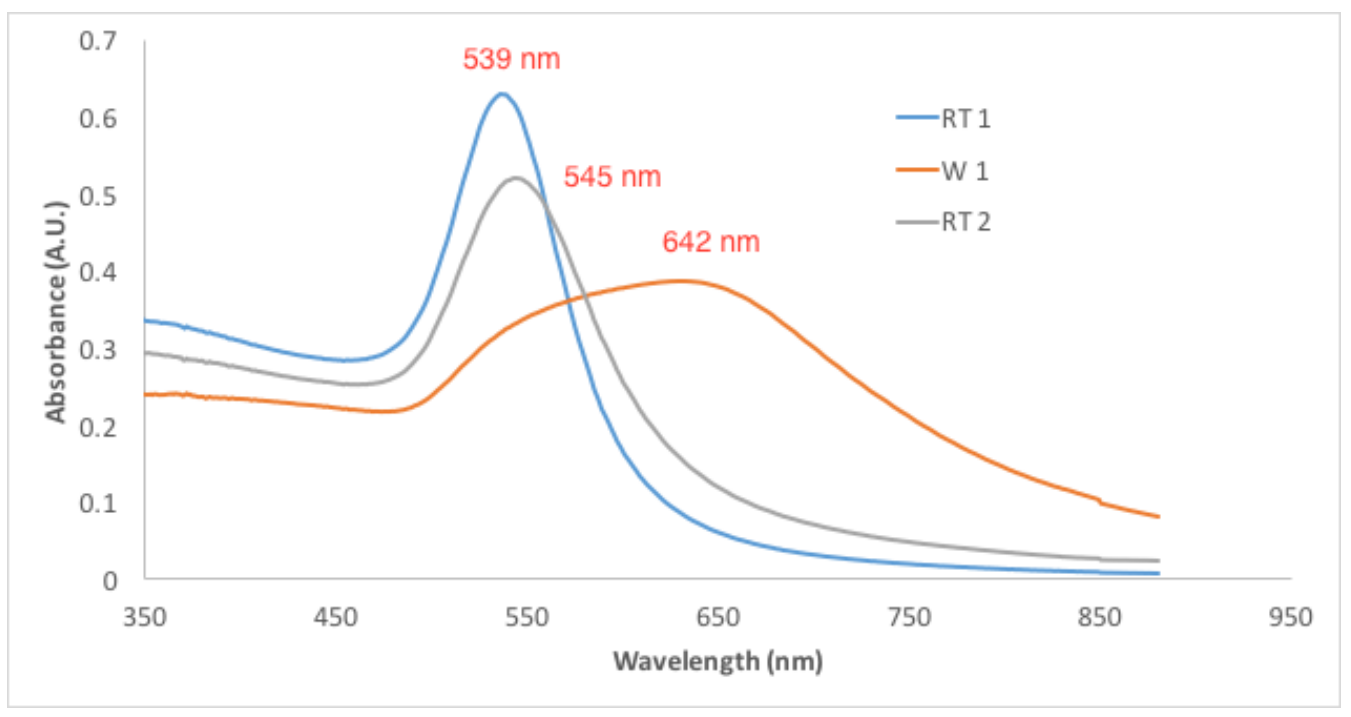

Figure 5.8 UV-VIS spectra before heating (RT 1), after heating (W 1), and after cooling (RT 2)

Similar results were found when using AgNPs where the solution darkened markedly from a light green to gray as seen in Figure 5.9. Returning to RT did not fully restore the original green hue as reflected by the UV-VIS absorbance spectra. Although a broad range of new resonance bands were created after heating, no distinct plasmon peak was formed which suggests aggregates with a greater range of size distribution, relative to that of AuNPs, had formed. 
Reversal of aggregation was also much less pronounced than that of gold. This may be due to the higher polydispersity of the silver colloid and/or the presence of non-spherical morphologies such as rods.

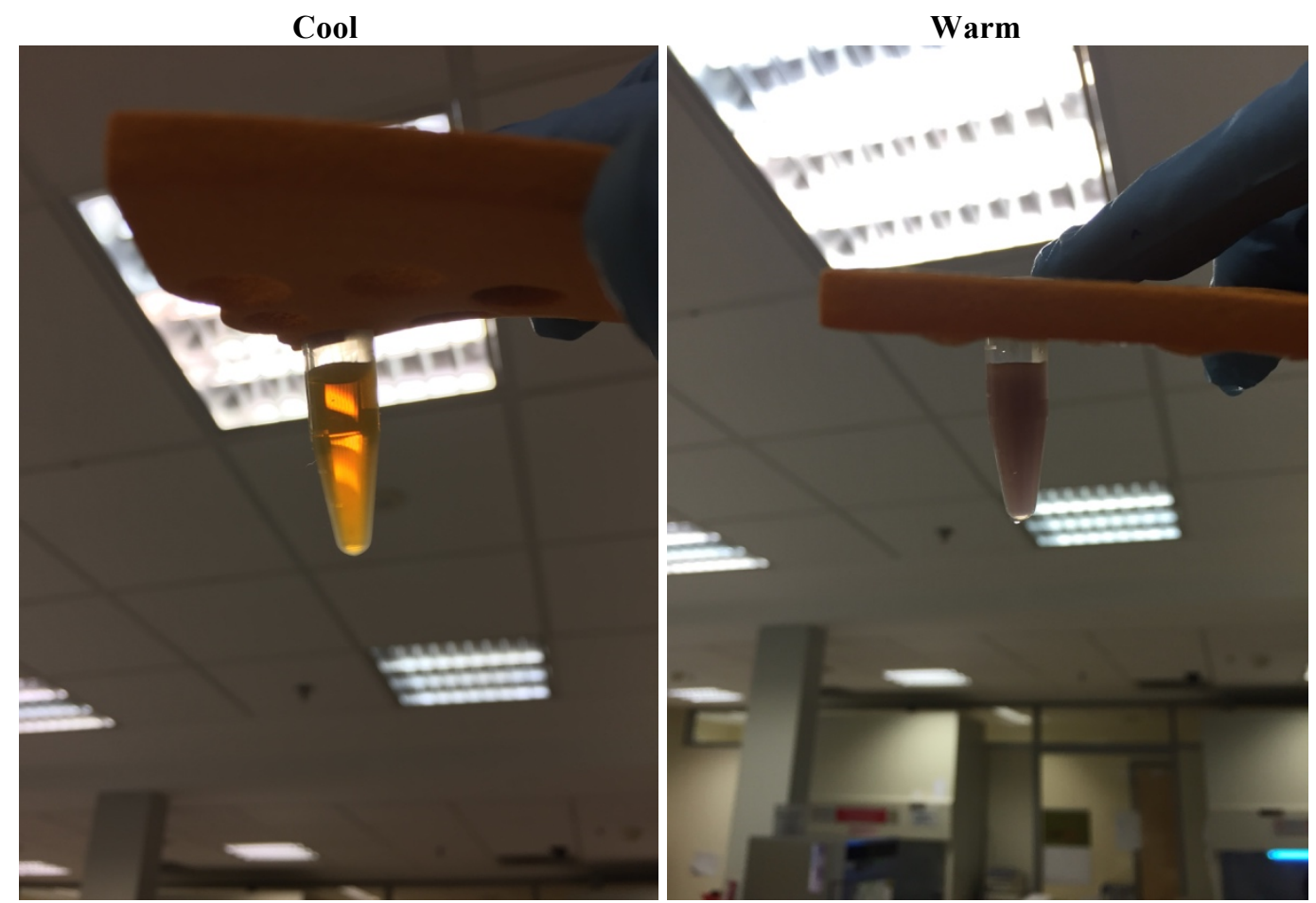

Figure 5.9 Heat induced aggregation of polyNIPAM functionalized AgNP.

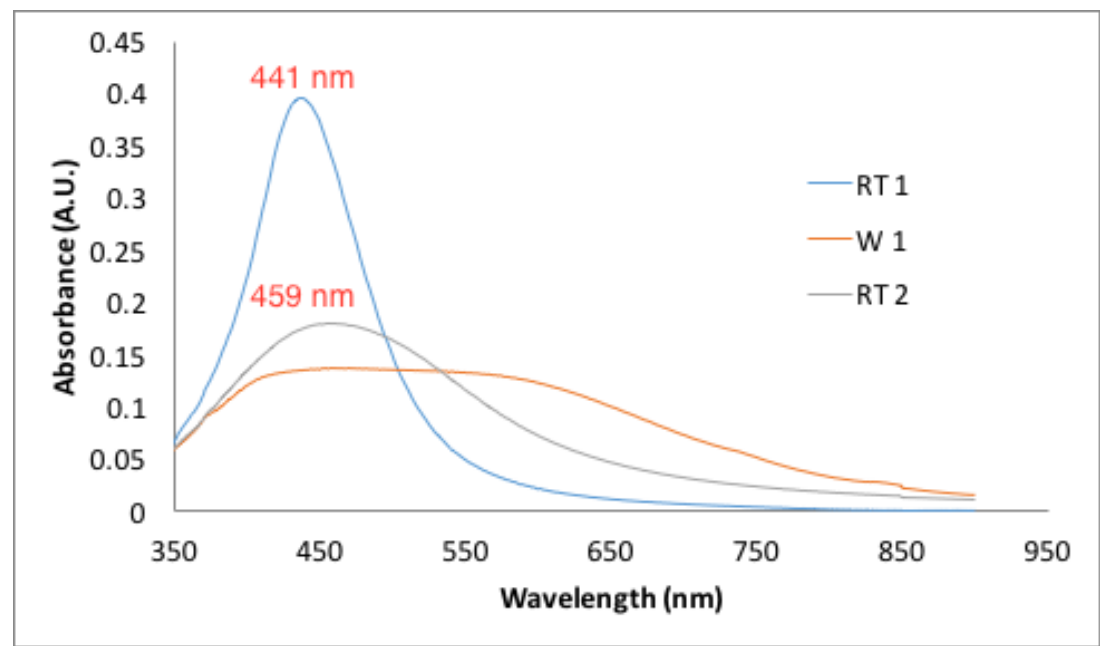

Figure 5.10 UV-VIS spectra of polyNIPAM functionalized AgNPs before heating (RT 1), after heating (W 1), after cooling (RT 2) 


\subsection{Modulated SERS in Colloid}

Modulated SERS was first attempted on AuNPs fully coated with polyNIPAMbiotin chains in $\mathrm{NaCl}$ solution $(50 \mathrm{mM})$. A small volume $(100 \mu \mathrm{L})$ was added to a small PCR tube that was suspended in a makeshift water bath depicted in Figure 5.11. An adjustable hotplate equipped with a feedback thermometer was set to $50{ }^{\circ} \mathrm{C}$ to raise the temperature of the sample. The sample was cooled by powering off the hotplate and replacing the warm water with ice-water via a pipette gun. Samples were allowed to equilibrate for two minutes after switching between cold and warm temperatures. Returning the water bath to 50 ${ }^{\circ} \mathrm{C}$ required approximately 15 minutes resulting in a total cold/hot cycle time of about 21 minutes when also accounting for the time needed to replace the water with ice-water. Samples were excited with about $10 \mathrm{~mW}$ at $633 \mathrm{~nm}$ with an integration time of 20 seconds. Spectra were recorded using an in-house optical setup connected to an Acton SP-2150i spectrometer coupled to a PIXIS 400 CCD (Princeton Instruments) with transmission efficiency optimized in the desired spectral range (633 to $725 \mathrm{~nm}$ ).

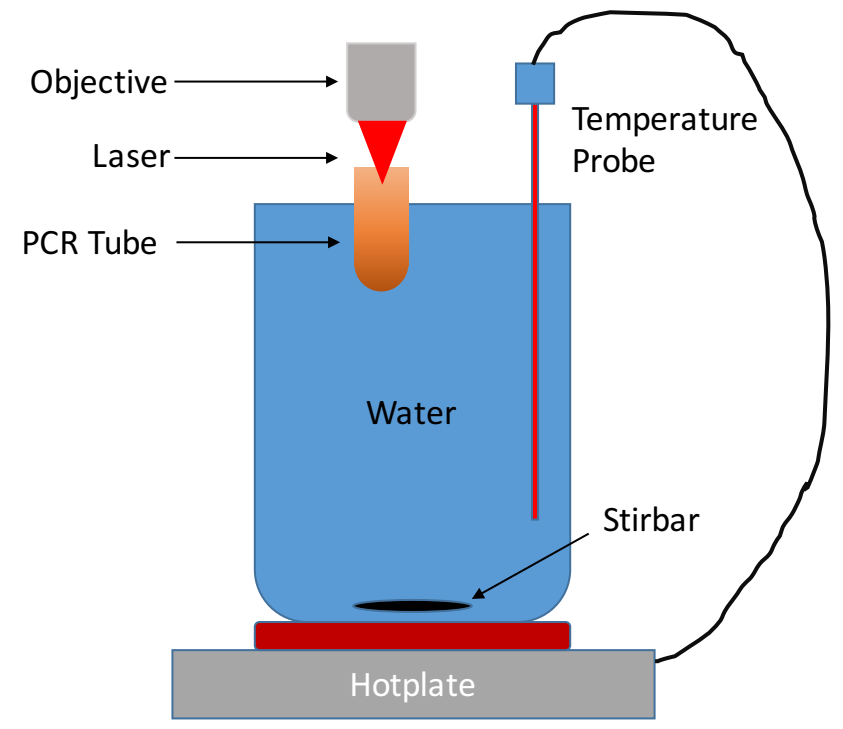

Figure 5.11 Makeshift water bath for modulated SERS 
Unfortunately, spectra of polyNIPAM functionalized AuNPs yielded no discernable peaks as seen in Figure 5.12. However, broad peaks centered at 480,808 , and $1415 \mathrm{~cm}^{-1}$ are observable. Raising the temperature and inducing an aggregated state did not cause the emergence of new peaks that may be related to chemical bonds within the polymer or to the biotin end group. The only change found was a slight increase in the fluorescent baseline without any change in spectral shape. Enhancement of fluorescence ${ }^{162}$ due to nanoparticle aggregation and/or infrared radiation emitted by the warm sample ${ }^{4}$ were likely to have caused this. Clearly, signals from the attached polymers were not enhanced as expected. More importantly, Raman peaks associated with the biotin end-group, which is the attachment site for the target protein, avidin, were absent.

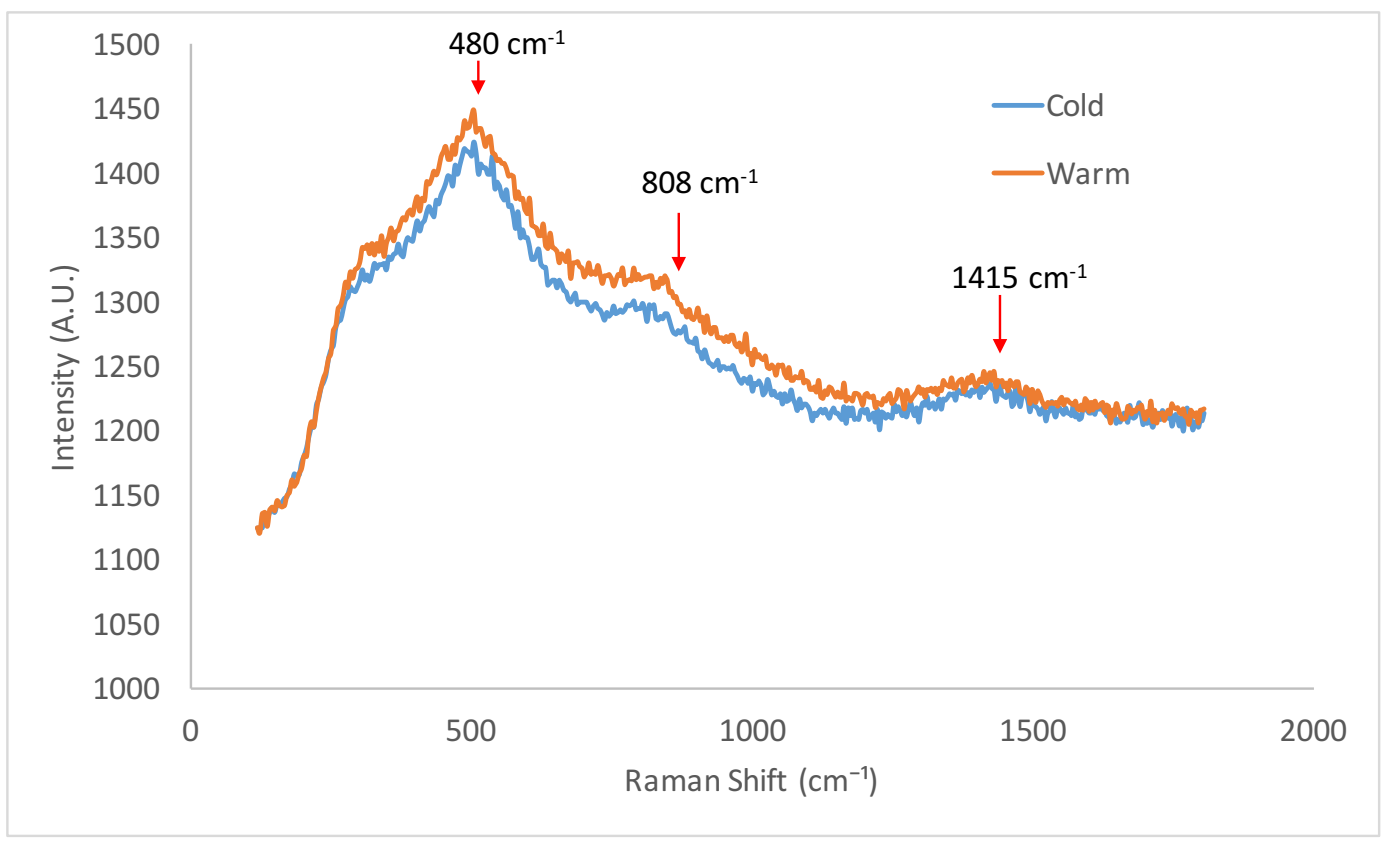

Figure 5.12 SERS spectra of polyNIPAM functionalized AuNP colloid before and after heating

It was conjectured that nanoparticle surfaces were overcrowded causing interference of surface plasmon excitation, which led us to explore smaller 
quantities. Unfortunately, using less polymer compromised the colloid's stability that was essential after centrifugation, to remove any unbound chains, which led to irrevocable aggregation. Attention was then turned to silver, however, AgNPs yielded similar results to that of gold as seen in Figure 5.13 even after increasing the exposure time up to 2 minutes.

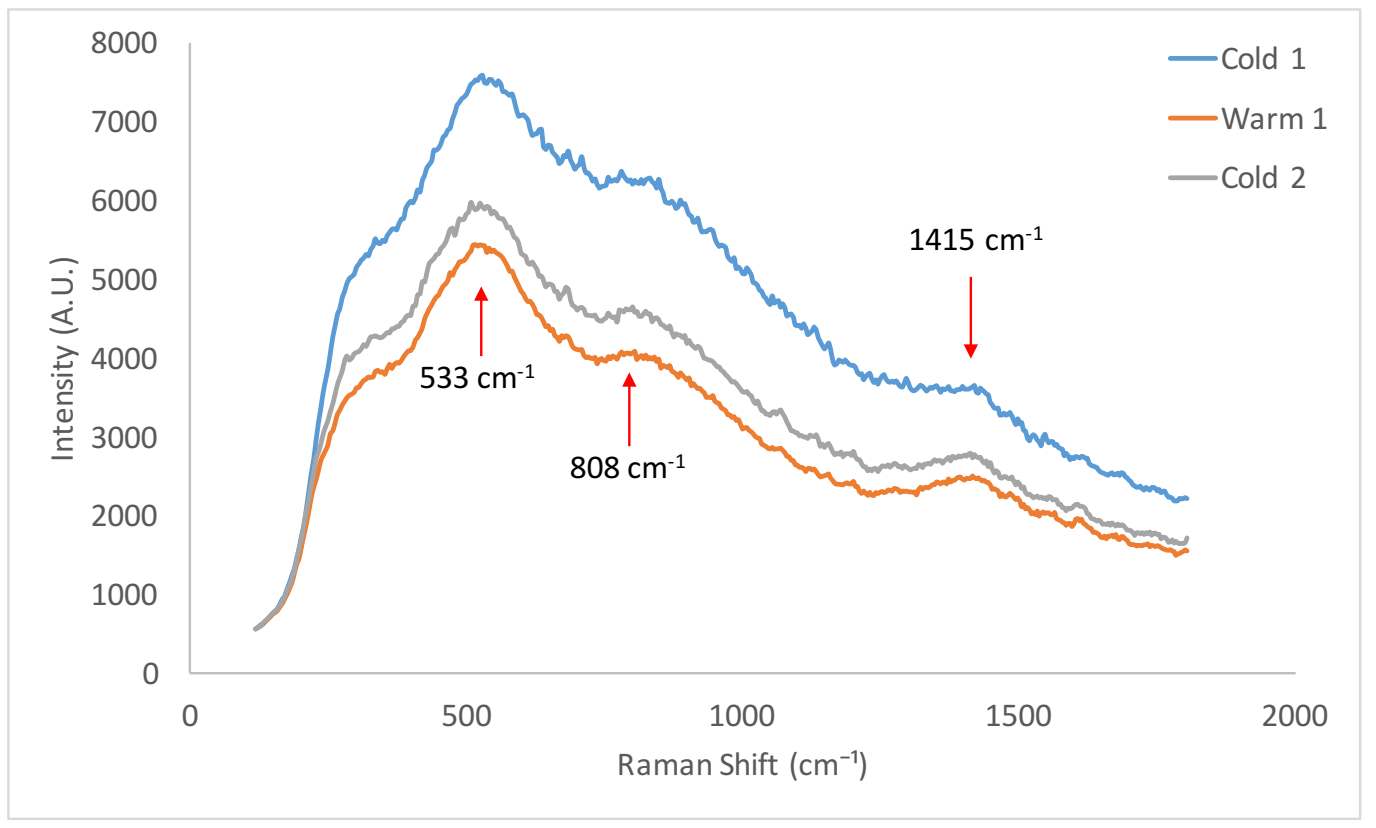

Figure 5.13 SERS spectra of polyNIPAM functionalized AgNP colloid before and after heating

The level of solution within the sample tube was found to decrease gradually as the experiment progressed possibly due to heating by the laser and the open exposure of the solution to the surroundings. Completing two cold/hot cycles required approximately 42 minutes, which was an impractical length of time in which the water had ample opportunity to spontaneously evaporate. Upon reevaluation of this approach, it was also determined that there was high potential for non-specific modulation of SERS since all proteins present in solution would be entrapped between nanoparticles after aggregation in exactly the same way as described in the previous chapter. The only difference was that aggregation could be reversed below the LCST. It was ultimately decided to 
abandon this approach in favor of immobilization of the nanoparticles onto the surface of a microscope slide. This approach eliminated the need for reversible aggregation and the delicate problem of maintaining colloid stability. The enhancing effect of aggregation, due to coupling of local EM fields, would still exist since the nanoparticles would be densely packed on the surface which is essentially a state of aggregation.

Another variable was the polymer chain length, which according to estimates by DLS, was too long such that the analyte could not come near enough to the plasmonic surface; ideally it would be less than $10 \mathrm{~nm}$ away. ${ }^{163}$ The overall diameter of $60 \mathrm{~nm}$ AuNPs fully saturated by polyNIPAM was estimated by DLS as the temperature was incremented from 4 to $34{ }^{\circ} \mathrm{C}$. The sample cuvette was allowed to reach equilibrium with the temperature adjustable sample blockholder for about two minutes for each temperature point. A clear trend was observed where the overall hydrodynamic diameter (HDD) began to decrease as the temperature approached the LCST approximately located at $32{ }^{\circ} \mathrm{C}$ as shown by the inlay in Figure 5.14. At $34{ }^{\circ} \mathrm{C}$, the colloid was completely destabilized and massive aggregates began forming causing an upsurge in the mean and standard deviation of HDD. Just before this tipping point, the approximate polymer length was calculated to be about $16 \mathrm{~nm}$. Although this approximation was calculated using the HDD which differs slightly from the actual diameter, we found it prudent to synthesize a shorter polymer chain to ensure exposure of the attached protein to the surface plasmon. To this end, polyNIPAM of DP=67 (now referred to as N67) was synthesized and used in all further experiments. 


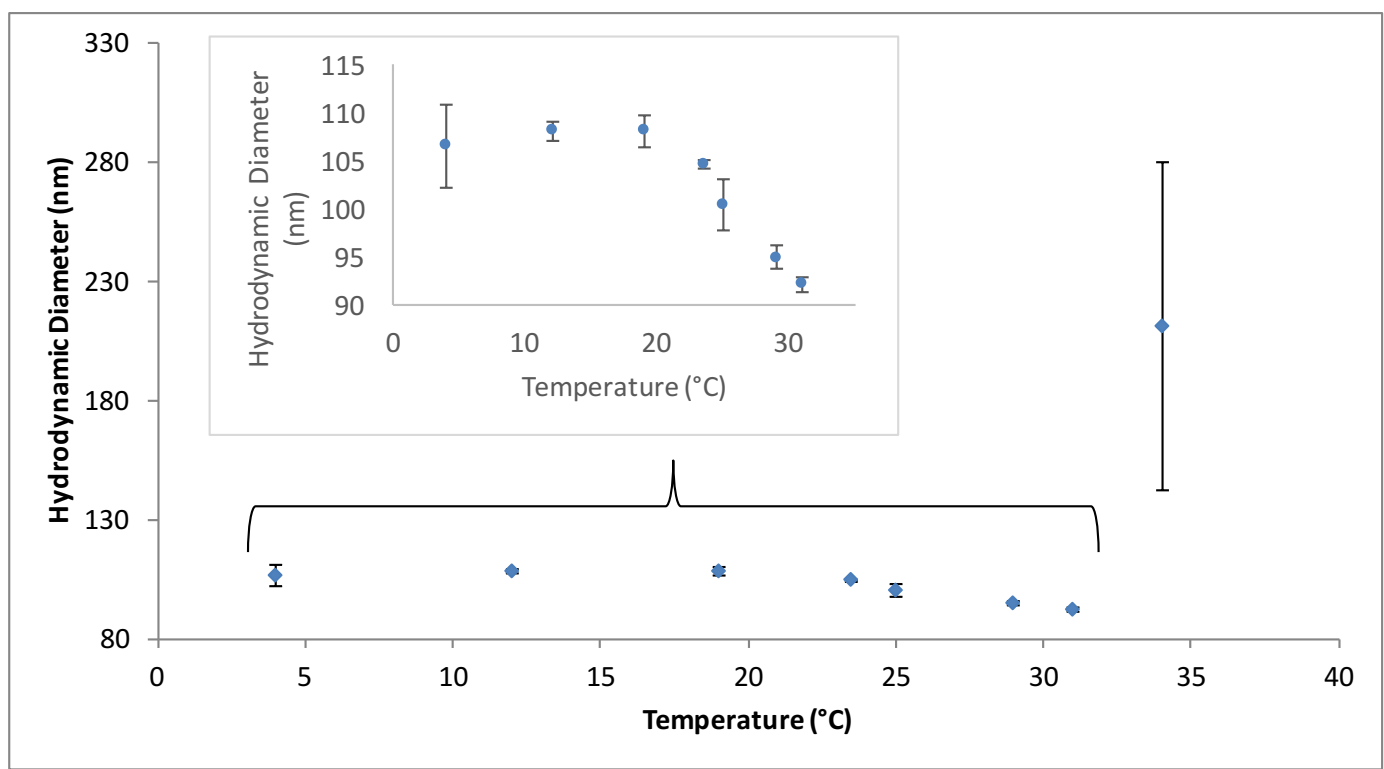

Figure 5.14 DLS polyNIPAM functionalized AuNPs as temperature was varied. Inlay: a closeup view of data points before aggregation to clearly see the gradual change in HDD with temperature

\subsection{AgNP Immobilization on Glass Substrate and Flow Cell Construction}

Glass coverslips were soaked in aqua regia for 15 minutes, rinsed with copious amounts of milliQ water, and dried in an oven. The coverslips were then plasma cleaned for five minutes to hydroxylate the surface followed by soaking in a solution of $N^{1}$-(3-Trimethoxysilylpropyl)diethylenetriamine $(5 \mathrm{mM})$ dissolved in toluene for 10 minutes and dried under nitrogen flow. See Figure 5.15 to view the preparation process. The silver colloid was rinsed and concentrated two-fold by centrifugation ( $3000 \mathrm{~g}$ for $30 \mathrm{~min}$ ) and $10 \mu \mathrm{L}$ of the resulting colloid was deposited onto each slide and allowed to dry overnight for nanoparticles to locate and bond with free amide groups. For this purpose, citrate-capped AgNPs were used for reasons thoroughly discussed in the previous chapter. 

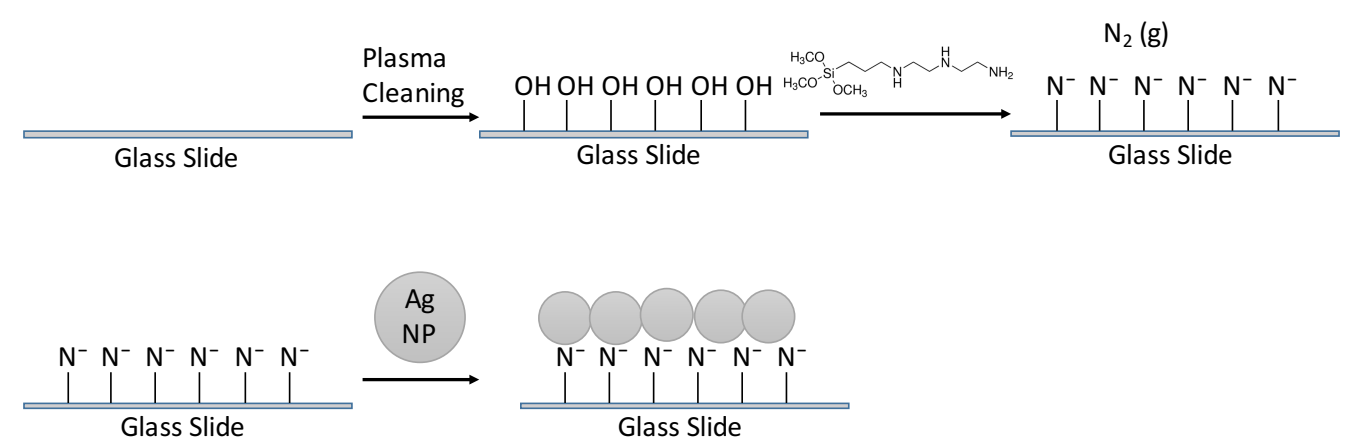

Figure 5.15 Preparation of immobilized AgNP surface

The surface was gently rinsed with Milli Q water to remove any unbound nanoparticles and finally dried in an oven. The circular patch of immobilized nanoparticles was enclosed within a flow cell by means of double sided tape and a quartz coverslip (see Figure 5.16). For a cross-sectional view, see Figure 5.17. Solution was initially drawn through the flow cell by capillary action and subsequently by absorbent cloth. Both open ends were sealed by grease during Raman measurement to prevent solution from leaving. The temperature was modulated by a custom made hollow-aluminum box through which warm and cold water could circulate by means of a peristaltic pump (see Figure 5.18). Warm water was maintained at $70^{\circ} \mathrm{C}$ using an adjustable hotplate and cold water was drawn from a separate beaker containing ice cubes. Switching between both sources required physically relocating the tubing to the desired source. The temperature of the metal box was estimated using an infrared thermometer. Direct measurement of the sample's temperature, however, could not be reliably ascertained by this method. 


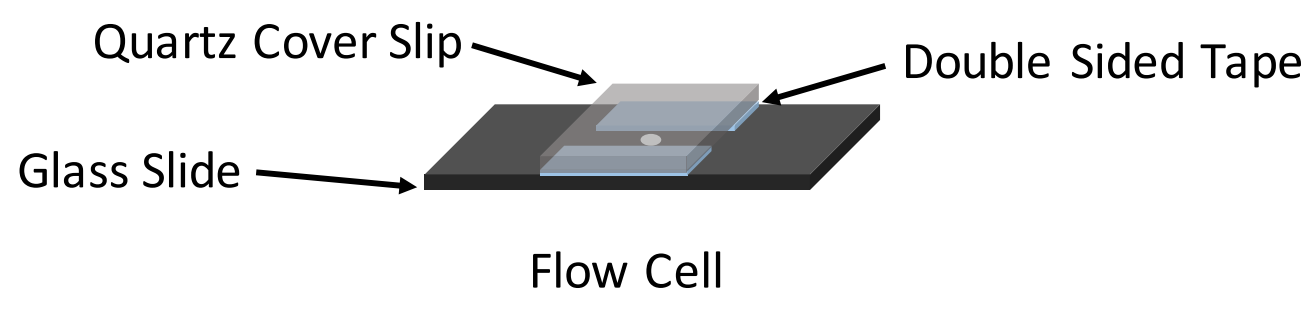

Figure 5.16 3D flow cell schematic

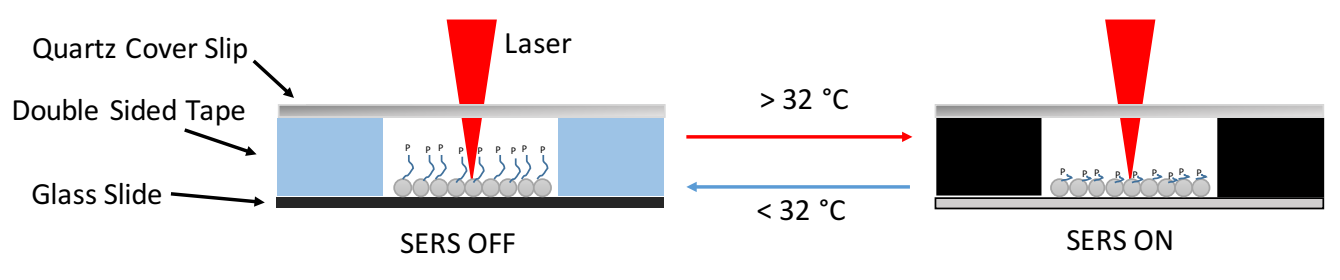

Figure 5.17 Flow cell cross-sectional view

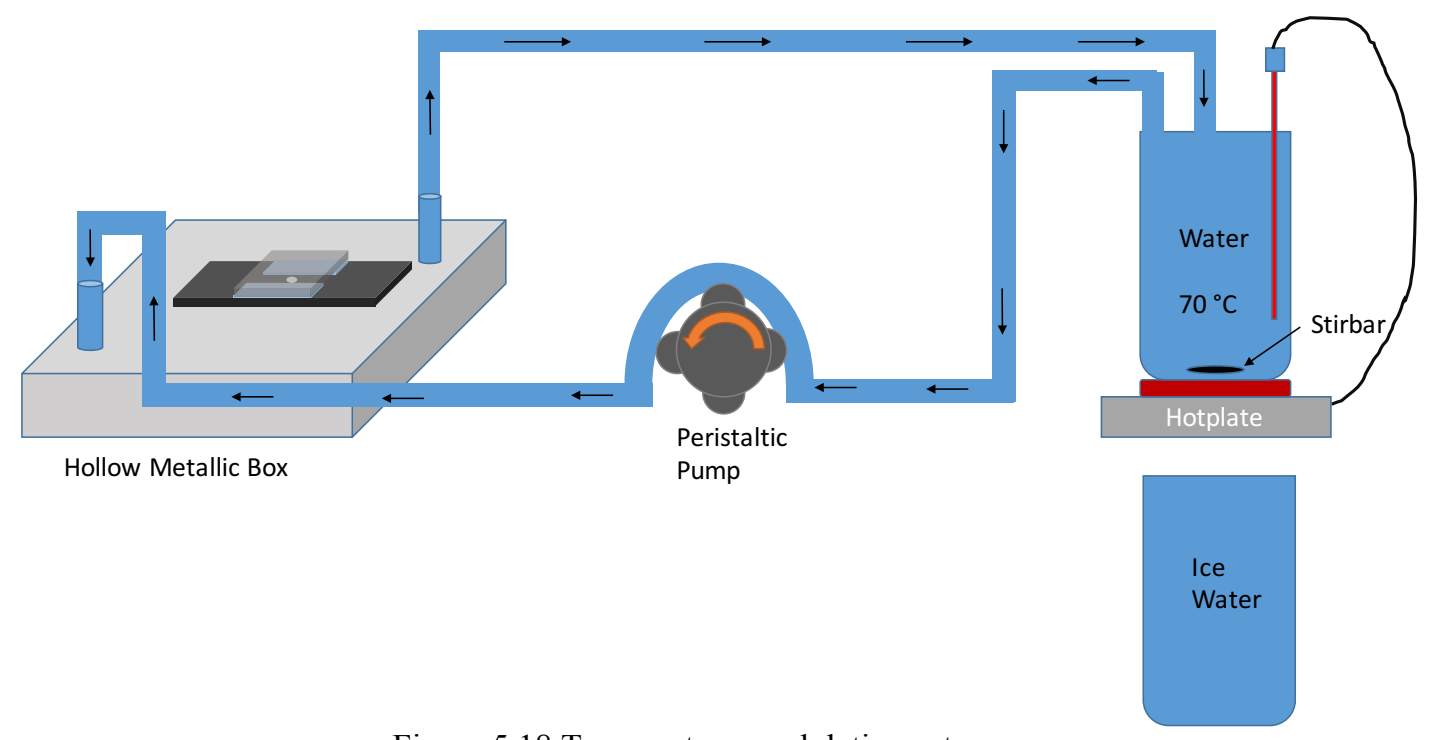

Figure 5.18 Temperature modulation setup

\subsection{Modulated SERS on Immobilized Nanoparticle Surface}

N67 $(1 \mathrm{mg} / \mathrm{mL})$ was added to the flow cell and allowed to incubate for 12 hours at $4{ }^{\circ} \mathrm{C}$ (to reduce the rate of evaporation and to maximize solubility for increased accessibility to the thiol end group). Subsequently, excess N67 was 
flushed out with Milli Q water followed by addition of a small molecule (SM) 2-mercaptoethanol $(0.03 \% \mathrm{w} / \mathrm{v}$ aqueous solution, $100 \mathrm{uL})$ to passivate any exposed nanoparticle surfaces; this was allowed to incubate for about 1 hour. The flow cell was sealed at both ends with grease and the surface was irradiated by the excitation laser source for Raman measurement. Samples required about ten minutes to equilibrate after switching temperatures followed by a Raman measurement using a 5 second exposure time and about $10 \mathrm{~mW}$ of power reaching the sample. This provided a detectable signal while minimizing the laser's thermal contribution. The temperature was considered stabilized after the temperature probe on the hotplate returned to the set temperature of $70{ }^{\circ} \mathrm{C}$, which required about 10 minutes. The temperature during the cooling phase was estimated by an infrared thermometer pointed at the metal box. During the equilibration phase, the laser beam was blocked to prevent it from being another heat source for the sample. Data was recorded for 14 hot/cold cycles. 


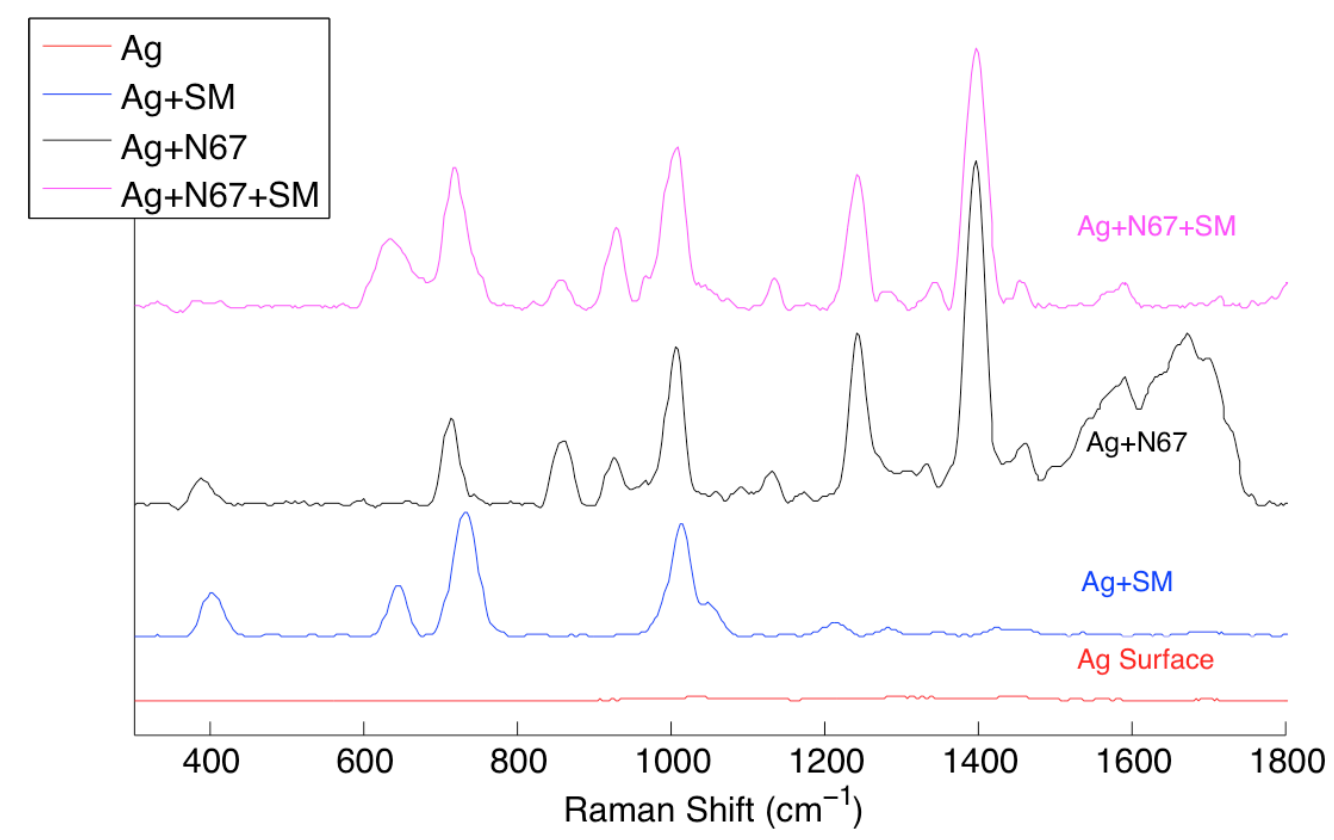

Figure 5.19 Individual spectra of bare $\mathrm{Ag}$ surface (red), Ag surface+SM (blue), Ag surface+N67 (black), and Ag surface+N67+SM (magenta)

Spectra of the bare silver surface $(\mathrm{Ag}), \mathrm{Ag}+\mathrm{SM}, \mathrm{Ag}+\mathrm{N} 67$, and $\mathrm{Ag}$ after treatment with both N67 and the SM are shown in Figure 5.19. The contribution of the SM can be seen at $632 \mathrm{~cm}^{-1}$ and indicated it did not totally displace bound N67 and that the surface was successfully passivated. The temperature of this sample was then modulated between cold and warm states and yielded the results found in Figure 5.20. Blue points indicate cool and red points indicate warm states of the selected peaks located at $632,720,852$, and $1403 \mathrm{~cm}^{-1}$. Since the SM is not temperature sensitive, there should be no change in its SERS intensity during temperature modulation. The expected increase in SERS intensity after heating and subsequent decrease after cooling was unfortunately not displayed by the remaining peaks. The data indicated no correlation between SERS intensity and solution temperature. 


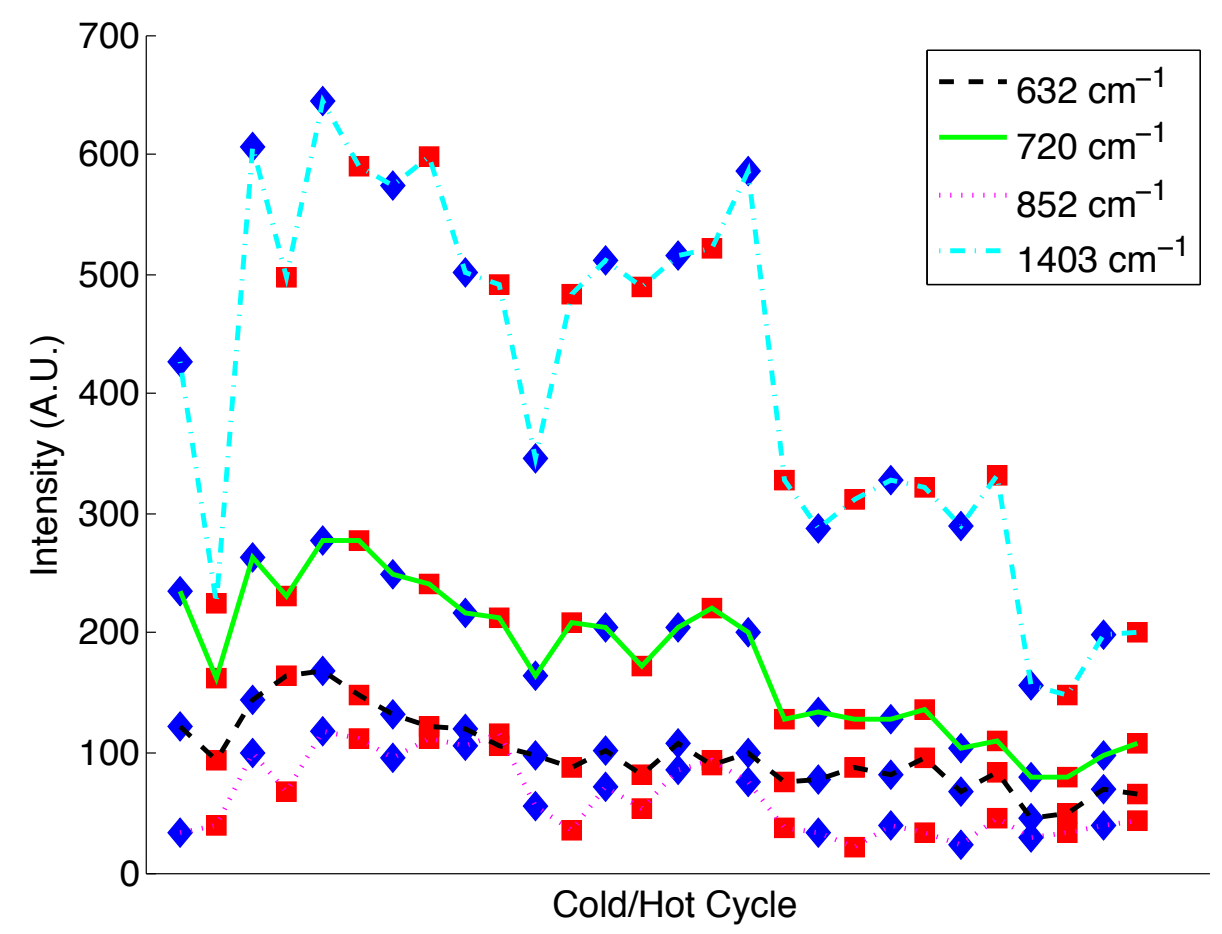

Figure 5.20 Band intensities of selected peaks at cold (blue marker) and warm (red marker) states

Despite no sign of modulated SERS, detection of avidin $(1 \mathrm{mg} / \mathrm{mL}, 100 \mathrm{uL})$ was attempted which was introduced after addition of the SM and allowed to incubate for 15 minutes at $4{ }^{\circ} \mathrm{C}$. Unbound avidin was flushed out using an excess of Milli Q water. Results displayed nearly no presence of the added avidin even after a longer incubation time of 30 minutes. Data shown in

Figure 5.21 is after 30 minutes of incubation using the same power and exposure time settings as mentioned earlier. Comparison of the protein by itself (in solution) and that of N67 shows they are spectrally similar making it difficult to discern the two. A single peak at $1540 \mathrm{~cm}^{-1}$ was observed that appeared to originate from the added avidin. However, the intensity of this band is relatively weak and would serve as the sole indicator of the protein's presence, which would be insufficient in more complex mixtures where other proteins would also make spectral contributions. In addition, the SERS intensity of this 
band did not show any increase after heating while other bands counterintuitively showed a decrease. In light of this, experimentation was discontinued.

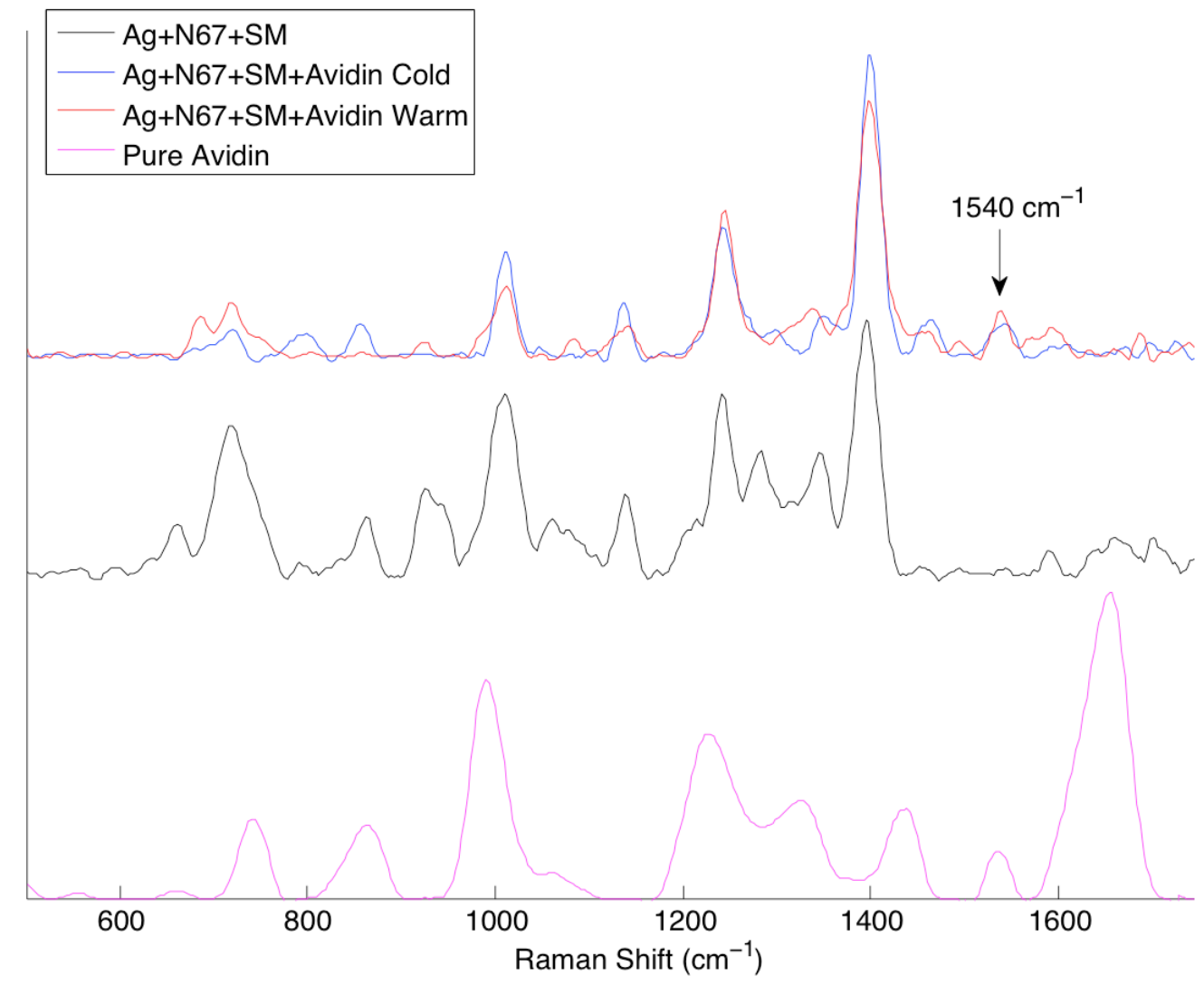

Figure 5.21 Raman spectra of Ag+N67+SM+Avidin Cold (top blue) and Ag+N67+SM+Avidin Warm (top red). Spectra of pure avidin and $\mathrm{Ag}+\mathrm{n} 67+\mathrm{SM}$ are also presented for comparison. 


\subsection{Discussion and Conclusion}

The use of a photosensitive and thermosensitive polymer for modulated SERS of protein was thoroughly explored. The original endeavor to develop and synthesize a photoresponsive polymer was quickly met with extraordinary obstacles that required a dedicated venue with sufficient resources for performing an unprecedented number of synthesis trials that is not possible in our current laboratory. Only in this way could we determine how to enable full polymerization (if at all possible), determine the correct ratio of hydrophilic to hydrophobic monomers (after accounting for the effect of biotinylation and the addition of a thiol end group), and find the optimum chain length for obtaining modulated SERS signals. The only definitive conclusion drawn from the experiments performed is that RAFT polymerization cannot reach completion using the acrylic acid and azobenzene monomers within DMF or dioxane that are common organic solvents in these types of synthesis reactions. ${ }^{164,} 165$ Unfortunately, the arrangement with ICES was limited and inflexible due in large part to the tight security measures imposed on Jurong Island that prohibits non-ICES personnel from remaining within the facility after business hours and during weekends. Furthermore, a dedicated fume hood for running the reactions was only available to full time ICES employees who took priority, which required us to wait until the person in charge had finished experiments. As a result, development of a photoresponsive polymer was deemed unworkable in such a setting and inapt within the limited time of a doctorate program. Even so, we were reasonably circumspect about proceeding further since this was likely an insurmountable challenge even if all the aforementioned resources were at hand. 
Switching to a thermoresponsive polymer avoided the complex chemistry challenges associated with engineering a novel polymer and greatly simplified our efforts to show a proof of concept. However, this approach's greatest drawback was the slow speed by which temperature can be modulated. Although the synthesis was straightforward, polyNIPAM in a colloidal solution proved ineffective in generating SERS signals as shown by recorded spectra taken in both the dispersed and aggregated states. Fortunately, a more robust arrangement using immobilized nanoparticles seemed to provide spectra with much greater detail. However, no repeatable modulation could be achieved most likely due to the inherent random coil conformation of polymer chains.

Despite the rehydration of the polymers below the LSCT, each chain would reexpand in an unpredictable manner as one can imagine how a random coil would behave in solution. One may visualize the situation where a polymer chain is anchored to an immobilized nanoparticle surface in aqueous solution. When transitioning to the hydrophobic state, the polymer agglomerates upon itself bringing more of its constituent monomers downward and closer to the nanoparticles. However, returning to the hydrophilic state does not guarantee it will return to its original conformation since the manner of re-expansion is unpredictable under the influence of Brownian motion and possibly uneven distribution of temperature. For example, a chain might re-expand in a more lateral manner leaving the majority of the length of each chain near the plasmonic surface and maintaining a strong SERS signal. Moreover, the close proximity between polymers and metal may incite bonding via the adsorption 
of nitrogen atoms present within each monomer subunit that may cause sticking to the surface and restrict modulation. In other instances, a chain may expand in a more vertical fashion, bringing most of the chain farther away from the surface. Brownian motion influencing the movement of these chains in the hydrophilic state was also not considered as it alone may cause erratic SERS signals. Furthermore, the idea of using a polymer chain is specious at best given the chemical similarity with biological polymers, in this case protein. Both molecules share the same basic chemical bonds such as amides and carboncarbon bonding. Therefore, it was not surprising to find high similarity between the spectra of polyNIPAM and that of pure avidin, which only hampered efforts in identifying the protein's signal. In principle, the SERS of both the polymer and the avidin would modulate, making them impossible to separate. Moreover, the simple fact that the polymer is nearest to the plasmonic surface would mean the polymer's SERS signal would be more intense than the that of the protein making detection more difficult.

Granted, one peak did appear to have originated from avidin, however, only one peak is the bare minimum required to positively identify avidin in the presence of a multitude of other proteins. Upon the addition of heat, this peak displayed no increase in intensity whatsoever. Perhaps another protein exhibiting peaks not shared by the polymer would allow us to investigate more peaks, but this would require chemically binding the antibody to the polymer, which is not only costly, but would be extremely difficult to purify since organic solvents that denature antibodies cannot be used. Using dialysis tubing is one possibility, however, this would need to be done in the absence of oxygen to prevent the 
formation of disulfide bonds among polymer chains. The shorter polyNIPAM polymer was attempted in a colloidal solution, however, its addition to nanoparticle solution led to irreversible aggregation, which is common when adding small stabilizing molecules such as mercaptoacetic acid directly. ${ }^{166}$ Ligand exchange was attempted using PEG2000, but the final colloid exhibited no change in aggregation before and after heating, which indicated N67 could not be successfully conjugated to the nanoparticles used. Please refer to Chapter 6 for a discussion of future work. 


\section{Chapter 6 Conclusions and Future Directions}

\subsection{Conclusions}

This dissertation presented three major studies utilizing Raman spectroscopy for the detection and quantification of proteins. All studies shared a common goal of achieving fast, minimally invasive and cost effective protein detection to overcome the disadvantages of current methods. Specifically, polarized Raman spectroscopy, Raman optical activity, and modulated SERS were explored. Most notably, this thesis has contributed to Raman based biomedical diagnostics by showing PRS, a technique exclusively used for structural analysis of crystalized substances, to be applicable in multiplexed protein quantification in solution. The following presents the conclusions of these studies.

The first study demonstrated how polarized Raman spectroscopy can be used to significantly enhance quantitative accuracy in an aqueous binary mixture containing $\alpha$-amylase and lysozyme. This was achieved by exploiting peaks whose intensity were found to have greater sensitivity to change in protein concentration when viewed from either the parallel or perpendicular configuration. Inputting these selected bands into non-linear least square regression not only showed increased quantitative accuracy but also reproducibility in comparison to traditional Raman spectroscopy. Since calculating the concentration of each protein was based on each protein component spectrum, this method was readily extensible to more complex mixtures by simply recording each component spectrum. If combined with Raman enhancement methods to access biologically relevant concentrations, 
polarized Raman spectroscopy may play an important role in future biomolecular detection platforms.

The second study is not at a stage at which many conclusions may be drawn. Grappling with the immense difficulty of constructing an ROA setup stood as the most complex phase of this work. However, a working setup was successfully constructed and the ROA spectra of (+)- $\alpha$-pinene were shown obtainable using $785 \mathrm{~nm}$ excitation without automated correction routines, but was found highly impractical given the correction required about three straight full days of trial and error of making minute adjustments. Although previous studies have published SERROA spectra of myoglobin and cytochrome c, it remains to be seen after implementing an electro-optic modulator for an automated correction routine if the SEROA of proteins is obtainable at $785 \mathrm{~nm}$ excitation.

Lastly, with respect to the creation of a photoresponsive polymer, it was determined that RAFT polymerization using the monomers and solvents discussed could not reach full polymerization beyond a DP of 20. Grappling with this problem was beyond our purview and the scope of our abilities given the limitations of our collaboration as well as the vast number of variables involved. Lack of a proof of concept and uncertainty of the solution to the polymerization problem gave strong motivation to conclude this approach.

Attention was turned to thermoresponsive polymers that are well known and, therefore, easy to synthesize. The results indicated this approach is unworkable 
since definitive spectra could not be obtained in a colloidal state. However, polyNIPAM was shown to effectively and reliably modulate the state of aggregation of gold nanoparticle colloids. With respect to SERS, although definitive spectra were obtainable, no dependence of signal intensity and temperature could be found. Moreover, given the high similarity between the polymer and protein chemical composition, our ability to identify the spectral contribution of the protein was severely impaired. These results strongly indicated some inherent flaws of this polymer-based approach especially when considering its random coil behavior which cannot repeatedly re-extend into solution with the same conformation. All results strongly indicated that the exploitation of thermoresponsiveness of polymers for achieving modulated SERS was unfeasible in both a colloidal system and on immobilized nanoparticles.

\subsection{Future Direction}

The natural future direction of the polarized Raman study described in Chapter 4 is to continue with the circularly polarized Raman setup to measure Surface Enhanced Raman Optical Activity. Two key modifications to the optical setup are reorientation to mimic an inverted microscope and implementation of an electro-optic modulator (EOM) for automated correction. Once completed, the SEROA spectra of proteins and protein mixtures will be obtained for enhanced quantification, relative to traditional Raman spectroscopy, of each protein species. Details are described as follows. 
The most important modification is the re-orientation of the optical train to resemble an inverted microscope. This enables the placement of the sample cuvette above the collecting lens so the aggregates that land on the bottom can be easily targeted for extended periods. A potentially more facile alternative is procuring a relatively flat cuvette measuring no more than a few millimeters in height to allow the laser focal volume to reach aggregates residing at the bottom. However, the presence of more stable aggregates may still drift about and cause transient fluctuations if they traverse the laser focal volume. In any case, fluctuations are bound to occur (largely owing to Brownian motion ${ }^{167}$ ), which demand the implementation of an automated correction routine that uses a nonresonant electro-optic modulator $(\mathrm{EOM})$ for rapid modulation between $\mathrm{CP}$ states. This would additionally allow us to fine-tune the incident polarization to minimize artifacts while also opening the possibility of implementing an automated self-correcting optical routine without which ROA is difficult to measure devoid of distortion when considering the re-adjustments required when interrogating new positions within the sample cuvette. ${ }^{6}$ Moreover, ambiguity of the local EM field polarization sensed by analytes due to irregularities among hot-spots could possibly be compensated by an EOM. ${ }^{6}$

Successful use of an EOM has been demonstrated in previous ICP-ROA reports where RCP and LCP incident radiation is modulated and electronically synchronized to a spectrometer. ${ }^{12,151,168}$ Modulation has been reported to be between ten and few hundred $\mathrm{Hz}$ to minimize the time between exposures of RCP and LCP states to reduce the influence of ephemeral fluctuations. ${ }^{169}$ All the respective exposures are summed to provide the final spectrum. To date, 
there is no known report of a modulation scheme in DCP-ROA for addressing the instability of colloidal aggregates, which would represent a new step forward in this field.

Once fine-tuning is complete and ROA of both (+)- $\alpha$-pinene and (-)- $\alpha$-pinene are successfully measured, the SEROA and parent SERS of various proteins will be measured individually and then in mixtures. The concentration of each protein within each mixture will be quantified using SEROA and SERS data to determine if using structurally sensitive SEROA spectra instead of chemically sensitive SERS spectra to deconvolute protein mixtures can significantly enhance quantitative accuracy.

The modulation mechanism based on polymer chains described in Chapter 5 was found unworkable, and has incited us to explore new modulation mechanisms. A potentially promising approach uses molecular motors that have been shown to generate rotary motion when exposed to light at the nanoscale. ${ }^{170}$ Such an approach, in principle, would not only enable highly reproducible modulation due to the precise nature of the motion generated, but also allows for protein specificity that is not possible in colloidal systems. Moreover, as the previous statement alluded to, the issues brought on by the behavior of random coil polymer chains that created sporadic enhancement rather than a consistently modulated one would be entirely avoided.

Given the rotary motion is isolated to a single plane, a surface comprised of immobilized nanoparticles would be too crudely structured to enable switching 
between a position when the analyte is distant from a plasmonic surface (creating low enhancement) and a position when it is near (creating high enhancement). Addressing this problem best involves the exploration and development of a highly ordered nanoscale plasmonic structure or micropatterned arrays such that the relative position and orientation of molecules and plasmonic surfaces are tightly controlled. Such surfaces have been fabricated by lithography methods common to semiconductor processing and other chemical means. $^{41,171-174}$

However, numerous obstacles still remain in terms of chemically modifying molecular motors with thiols or other binding groups for adsorption to a plasmonic surface. More importantly are the chemical modification for binding to protein analytes, if at all possible. Such changes may disrupt the motors' function, and would require extensive research and experimentation to determine the feasibility of this approach. 


\section{Author's Publications}

Academic Journals

1. Perlaki, C. M., Liu, Q., \& Lim, M. (2014). Raman Spectroscopy Based Techniques in Tissue Engineering-An Overview. Applied Spectroscopy Reviews, 49(7), 513-532.

2. Perlaki, C., Lim, S., \& Liu, Q. (2015). Polarized Raman spectroscopy for enhanced quantification of protein concentrations in an aqueous mixture. Journal of Raman Spectroscopy, 46(9), 744-749.

3. Chen, K., Perlaki, C., Xiong, A., Preiser, P., \& Liu, Q. (2016). Review of Surface Enhanced Raman Spectroscopy for Malaria Diagnosis and a New Approach for Detection of Single Parasites in the Ring Stage. IEEE Journal of Selected Topics in Quantum Electronics, 22(4), 6900509.

4. Wei, D., Chen, S., Hong, YH., Perlaki, C., \& Liu, Q. (2016) Fast wide-field Raman spectroscopy imaging based on simultaneous multi-channel image acquisition and Weiner estimation. Optics Letters, 41(12), 2783-2786

5. Liu, W., Ong, YH., Yu, XJ., Ju,J., Perlaki, CM., Liu, LB., \& Liu, Q. (2016) Snapshot depth sensitive Raman spectroscopy in layered tissues. Optics Express, 24, 28312-28325

Conferences and Presentations

1. Perlaki, C., Lim, S., \& Liu, Q. (2014) Polarized Raman Spectroscopy for Enhanced Protein Quantification in a Binary Mixture. Paper presented at the $6^{\text {th }}$ International Symposium on Microchemistry and Microsystems, Singapore

2. Perlaki, C., \& Liu, Q. (2016) Surface Enhanced Raman optical activity for characterization of aqueous protein mixtures. Paper presented at SPIE Photonics West, San Francisco, CA

3. Liu, Q. Presented by Perlaki, C. (2016) Development of Fast Spectroscopic Raman Imaging for Biomedical Applications. Paper presented at AsiaPacific Laser Symposium, Jeju Island, South Korea 


\section{References}

1.Ferraro JR, Nakamoto K, Brown CW. Introductory Raman spectroscopy. 2nd ed. Amsterdam ; Boston: Academic Press; 2003.

2.Koningstein JA. Introduction to the theory of the Raman effect. Dordrecht,: Reidel; 1972.

3.Long DA. The Raman effect : a unified treatment of the theory of Raman scattering by molecules. Chichester ; New York: Wiley; 2002.

4.Vandenabeele P. Practical Raman spectroscopy : an introduction. The Atrium, Southern Gate, Chichester, West Sussex, United Kingdom: Wiley; 2013.

5.Larkin P. Infrared and Raman Spectroscopy; Principles and Spectral Interpretation: Elsevier Science; 2011.

6.Nafie LA. Vibrational optical activity : principles and applications. Chichester, West Sussex: Wiley; 2011.

7.Wen ZQ, Hecht L, Barron LD. Beta-Sheet and Associated Turn Signatures in Vibrational Raman Optical-Activity Spectra of Proteins. Protein Sci. 1994;3(3).

8.Barron LD. Molecular light scattering and optical activity. 2nd ed. Cambridge, UK ; New York: Cambridge University Press; 2004.

9.Barron LD, Wen ZQ, Hecht L. Vibrational Raman Optical-Activity of Proteins. Journal of the American Chemical Society. 1992;114(2).

10.Barron LD, Hecht L, Ford SJ, Bell AF, Wilson G. Vibrational Raman Optical-Activity Biopolymers. J Mol Struct. 1995;349.

11.Barron LD, Escribano JR. Stokes-Antistokes Asymmetry in Natural Raman Optical-Activity. Chem Phys. 1985;98(3).

12.Barron LD, Buckingham AD. Vibrational optical activity. Chem Phys Lett. 2010;492(4-6).

13.Barron LD. From cosmic chirality to protein structure: Lord Kelvin's legacy. Chirality. 2012;24(11).

14.Barron LD, Vrbancich J. Natural vibrational Raman optical activity. Structural Chemistry. Berlin, Heidelberg: Springer Berlin Heidelberg; 1984. p. 151-82.

15.Barron LD, Zhu FJ, Hecht L. Raman optical activity: An incisive probe of chirality, and of biomolecular structure and behaviour. Vib Spectrosc.

2006;42(1). 
16.Li HG, Nafie LA. Simultaneous acquisition of all four forms of circular polarization Raman optical activity: results for a-pinene and lysozyme. $J$ Raman Spectrosc. 2012;43(1).

17.Yu GS, Nafie LA. Isolation of Preresonance and out-of-Phase Dual CircularPolarization Raman Optical-Activity. Chem Phys Lett. 1994;222(4).

18.Yu G-S, Freedman TB, Nafie LA. Dual circular polarization Raman optical activity of related terpene molecules: Comparison of backscattering DCPI and right-angle ICP spectra. J Raman Spectrosc. 1995;26(8-9).

19.Spencer KM, Freedman TB, Nafie LA. Scattered circular polarization Raman optical activity. Chem Phys Lett. 1988;149(4).

20.Abdali S, Blanch EW. Surface enhanced Raman optical activity (SEROA). Chem Soc Rev. 2008;37(5).

21.Abdali S. Observation of SERS effect in Raman optical activity, a new tool for chiral vibrational spectroscopy. J Raman Spectrosc. 2006;37(12).

22.Barron LD, Hecht L, Blanch EW, Bell AF. Solution structure and dynamics of biomolecules from Raman optical activity. Prog Biophys Mol Bio.

2000;73(1).

23. Mutter ST, Pour SO, Blanch EW. Raman Optical Activity. Encyclopedia of Analytical Chemistry: John Wiley \& Sons, Ltd; 2006.

24.Hecht L, Jordanov B, Schrader B. Mueller,Stokes Treatment of Artifacts in Natural Raman Optical-Activity. Appl Spectrosc. 1987;41(2).

25.Che DP, Nafie LA. Theory and Reduction of Artifacts in Incident, Scattered, and Dual Circular-Polarization Forms of Raman Optical-Activity. Appl Spectrosc. 1993;47(5).

26.Berova N, Nakanishi Kj, Woody R. Circular dichroism : principles and applications. 2nd ed. New York: Wiley-VCH; 2000.

27.Hug W. Optical Artifacts and Their Control in Raman Circular Difference Scattering Measurements. Appl Spectrosc. 1981;35(1).

28.Hug W. Virtual Enantiomers as the Solution of Optical Activity's Deterministic Offset Problem. Appl Spectrosc. 2003;57(1).

29.Kneipp K, Kneipp H, Kneipp J. Surface-enhanced Raman scattering in local optical fields of silver and gold nanoaggregates-from single-molecule Raman spectroscopy to ultrasensitive probing in live cells. Acc Chem Res. 2006;39(7).

30.Qian X, Peng XH, Ansari DO, Yin-Goen Q, Chen GZ, Shin DM, et al. In vivo tumor targeting and spectroscopic detection with surface-enhanced Raman nanoparticle tags. Nat Biotechnol. 2008;26(1). 
31.Polubotko AM. The dipole-quadrupole theory of surface enhanced Raman scattering. New York: Nova Science Publishers; 2009.

32.Campion A, Kambhampati P. Surface-enhanced Raman scattering. Chemical Society Reviews. 1998;27(4).

33. Sharma B, Frontiera RR, Henry A-I, Ringe E, Van Duyne RP. SERS: Materials, applications, and the future. Materials Today. 2012;15(1-2).

34.Aroca R. Surface enhanced vibrational spectroscopy. Hoboken, NJ: Wiley; 2006.

35.Amendola V, Meneghetti M. Size Evaluation of Gold Nanoparticles by UVvis Spectroscopy. J Phys Chem C. 2009;113(11).

36.Xu LJ, Zong C, Zheng XS, Hu P, Feng JM, Ren B. Label-Free Detection of Native Proteins by Surface-Enhanced Raman Spectroscopy Using IodideModified Nanoparticles. Analytical chemistry. 2014;86(4).

37.Dong SY, Wang G, Wang W, Zhang Z, Zheng J. The role of cluster in surface-enhanced raman scattering (SERS). Applied Physics B. 1989;49(6).

38.Fornasiero D, Grieser F. Analysis of the visible absorption and SERS excitation spectra of silver sols. The Journal of Chemical Physics. 1987;87(5).

39.Laserna JJ, Cabalin LM, Montes R. Effect of substrate optical absorption on surface-enhanced Raman spectrometry on colloidal silver. Analytical chemistry. 1992;64(17)

40.Munro CH, Smith WE, Garner M, Clarkson J, White PC. Characterization of the Surface of a Citrate-Reduced Colloid Optimized for Use as a Substrate for Surface-Enhanced Resonance Raman Scattering. Langmuir. 1995;11(10).

41.Gunnarsson L, Bjerneld EJ, Xu H, Petronis S, Kasemo B, Kall M. Interparticle coupling effects in nanofabricated substrates for surface-enhanced Raman scattering. Appl Phys Lett. 2001;78(6).

42.Su KH, Wei QH, Zhang X, Mock JJ, Smith DR, Schultz S. Interparticle coupling effects on plasmon resonances of nanogold particles. Nano Letters. 2003;3(8).

43.Wang M, Benford M, Jing N, Cote G, Kameoka J. Optofluidic device for ultra-sensitive detection of proteins using surface-enhanced Raman spectroscopy. Microfluid Nanofluid. 2009;6(3).

44.Garcia-Leis A, Garcia-Ramos JV, Sanchez-Cortes S. Silver Nanostars with High SERS Performance. J Phys Chem C. 2013;117(15). 
45.Han XX, Huang GG, Zhao B, Ozaki Y. Label-Free Highly Sensitive Detection of Proteins in Aqueous Solutions Using Surface-Enhanced Raman Scattering. Analytical chemistry. 2009;81(9).

46.Martin I, Smith T, Wendt D. Bioreactor-based roadmap for the translation of tissue engineering strategies into clinical products. Trends in biotechnology. 2009;27(9).

47.Lim M, Ye H, Panoskaltsis N, Drakakis EM, Yue X, Cass AEG, et al. Intelligent bioprocessing for haemotopoietic cell cultures using monitoring and design of experiments. Biotechnology advances. 2007;25(4).

48.Plazcek MR, Chung I-M, Macedo HM, Ismail S, Mortera-Blanco T, Lim M, et al. Stem cell bioprocessing: Fundamentals and principles. Journal of the Royal Society, Interface. 2008;6(32).

49.Jell G, Swain R, M. Stevens M. Raman Spectroscopy: A Tool for Tissue Engineering. In: Matousek P, Morris MD, editors. Emerging Raman Applications and Techniques in Biomedical and Pharmaceutical Fields. Biological and Medical Physics, Biomedical Engineering. London, UK: Springer Berlin Heidelberg; 2010. p. 419-37.

50.Mather ML, Morgan SP, Crowe JA. Meeting the needs of monitoring in tissue engineering. Regenerative medicine. 2007;2(2).

51.Owen Ca, Notingher I, Hill R, Stevens M, Hench LL. Progress in Raman spectroscopy in the fields of tissue engineering, diagnostics and toxicological testing. Journal of Materials Science: Materials in Medicine. 2006;17(11).

52.Scadden DT. The stem-cell niche as an entity of action. Nature. 2006;441(7097).

53. Thomas GJ, Jr. Raman spectroscopy of protein and nucleic acid assemblies. Annu Rev Biophys Biomol Struct. 1999;28.

54.Tuma R. Raman spectroscopy of proteins: from peptides to large assemblies. Journal of Raman Spectroscopy. 2005;36(4).

55.Dukor RK. Vibrational Spectroscopy in the Detection of Cancer. In: Chalmers JM, Griffiths PR, editors. Handbook of Vibrational Spectroscopy. Chichester, UK: John Wiley \& Sons, Ltd; 2002. p. 3335-61.

56.Movasaghi Z, Rehman S, Rehman Iu. Raman Spectroscopy Can Detect and Monitor Cancer at Cellular Level: Analysis of Resistant and Sensitive Subtypes of Testicular Cancer Cell Lines. Applied Spectroscopy Reviews. 2012;47(7).

57.Pascut FC, Kalra S, George V, Welch N, Denning C, Notingher I. Noninvasive label-free monitoring the cardiac differentiation of human embryonic stem cells in-vitro by Raman spectroscopy. Biochimica et biophysica acta. $2013 ; 1830(6)$. 
58.Pascut FC, Goh HT, Welch N, Buttery LD, Denning C, Notingher I.

Noninvasive detection and imaging of molecular markers in live cardiomyocytes derived from human embryonic stem cells. Biophysical journal. 2011;100(1).

59.Chan JW, Lieu DK, Huser T, Li RA. Label-free separation of human embryonic stem cells (hESCs) and their cardiac derivatives using Raman spectroscopy. Analytical chemistry. 2009;81(4).

60. Schulze HG, Konorov SO, Caron NJ, Piret JM, Blades MW, Turner RFB. Assessing differentiation status of human embryonic stem cells noninvasively using Raman microspectroscopy. Analytical chemistry. 2010;82(12).

61.Downes A, Mouras R, Bagnaninchi P, Elfick A. Raman spectroscopy and CARS microscopy of stem cells and their derivatives. Journal of Raman spectroscopy JRS. 2011;42(10).

62.Uzunbajakava N, Lenferink a, Kraan Y, Volokhina E, Vrensen G, Greve J, et al. Nonresonant confocal Raman imaging of DNA and protein distribution in apoptotic cells. Biophysical journal. 2003;84(6).

63.Uzunbajakava N, Lenferink a, Kraan Y, Willekens B, Vrensen G, Greve J, et al. Nonresonant Raman imaging of protein distribution in single human cells. Biopolymers. 2003;72(1).

64. Shamsaie A, Jonczyk M, Sturgis J, Paul Robinson J, Irudayaraj J. Intracellularly grown gold nanoparticles as potential surface-enhanced Raman scattering probes. J Biomed Opt. 2007;12(2).

65.Liu Z, Hu C, Li S, Zhang W, Guo Z. Rapid intracellular growth of gold nanostructures assisted by functionalized graphene oxide and its application for surface-enhanced Raman spectroscopy. Analytical chemistry. 2012;84(23).

66. Miller CE, Thompson RP, Bigelow MR, Gittinger G, Trusk TC, Sedmera D. Confocal imaging of the embryonic heart: how deep? Microscopy and microanalysis : the official journal of Microscopy Society of America, Microbeam Analysis Society, Microscopical Society of Canada. 2005;11(3).

67.Bonnier F, Knief P, Meade aD, Dorney J, Bhattacharya K, Lyng FM, et al., editors. Collagen matrices as an improved model for in vitro study of live cells using Raman microspectroscopy. Clinical and Biomedical Spectroscopy and Imaging II; 2011 May 24-26; Munich, Germany.

68.Jones JR, Vats A, Notingher I, Gough JE, Tolley NS, Polak JM, et al. In Situ Monitoring of Chondrocyte Response to Bioactive Scaffolds Using Raman Spectroscopy. Key Engineering Materials. 2005;284-286.

69.Kunstar A, Otto C, Karperien M, van Blitterswijk C, van Apeldoorn A. Raman microspectroscopy: a noninvasive analysis tool for monitoring of 
collagen-containing extracellular matrix formation in a medium-throughput culture system. Tissue engineering Part C, Methods. 2011;17(7).

70.Kunstar A, Leferink AM, Okagbare PI, Morris MD, Roessler BJ, Otto C, et al. Label-free Raman monitoring of extracellular matrix formation in threedimensional polymeric scaffolds. Journal of the Royal Society, Interface / the Royal Society. 2013;10(86).

71.Nandagawali ST, Yerramshetty JS, Akkus O. Raman imaging for quantification of the volume fraction of biodegradable polymers in histological preparations. Journal of biomedical materials research Part A. 2007;82(3).

72.Moimas L, De Rosa G, Sergo V, Schmid C. Bioactive porous scaffolds for tissue engineering applications: investigation on the degradation process by Raman spectroscopy and scanning electron microscopy. Journal of applied biomaterials \& biomechanics : JABB. 2006;4(2).

73.van Apeldoorn AA, van Manen HJ, Bezemer JM, de Bruijn JD, van Blitterswijk CA, Otto C. Raman imaging of PLGA microsphere degradation inside macrophages. Journal of the American Chemical Society. 2004;126(41).

74.Luk JZ, Cooper-White J, Rintoul L, Taran E, Grondahl L. Functionalised polycaprolactone films and 3D scaffolds via gamma irradiation-induced grafting. Journal of Materials Chemistry B. 2013;1(33).

75.Rim NG, Kim SJ, Shin YM, Jun I, Lim DW, Park JH, et al. Mussel-inspired surface modification of poly(L-lactide) electrospun fibers for modulation of osteogenic differentiation of human mesenchymal stem cells. Colloids and surfaces B, Biointerfaces. 2012;91.

76.Jain S, Sharma A, Basu B. In vitro cytocompatibility assessment of amorphous carbon structures using neuroblastoma and Schwann cells. Journal of biomedical materials research Part B, Applied biomaterials. 2013;101(4).

77.Han XX, Kitahama Y, Tanaka Y, Guo J, Xu WQ, Zhao B, et al. Simplified protocol for detection of protein-ligand interactions via surface-enhanced resonance Raman scattering and surface-enhanced fluorescence. Analytical chemistry. 2008;80(17).

78.Han XX, Chen L, Guo J, Zhao B, Ozaki Y. Coomassie brilliant dyes as surface-enhanced Raman scattering probes for protein-ligand recognitions. Analytical chemistry. 2010;82(10).

79.Movasaghi Z, Rehman S, Rehman IU. Raman Spectroscopy of Biological Tissues. Applied Spectroscopy Reviews. 2007;42(5).

80.Gong Q, Wang J, Ahmad KM, Csordas AT, Zhou J, Nie J, et al. Selection strategy to generate aptamer pairs that bind to distinct sites on protein targets. Analytical chemistry. 2012;84(12). 
81.Wang Y, Wei H, Li B, Ren W, Guo S, Dong S, et al. SERS opens a new way in aptasensor for protein recognition with high sensitivity and selectivity. Chemical communications (Cambridge, England). 2007(48).

82.Fabris L, Dante M, Nguyen T-Q, Tok JBH, Bazan GC. SERS Aptatags: New Responsive Metallic Nanostructures for Heterogeneous Protein Detection by Surface Enhanced Raman Spectroscopy. Advanced Functional Materials. 2008;18(17).

83.Fabris L, Schierhorn M, Moskovits M, Bazan GC. Aptatag-based multiplexed assay for protein detection by surface-enhanced Raman spectroscopy. Small (Weinheim an der Bergstrasse, Germany). 2010;6(14).

84.Pagba CV, Lane SM, Cho H, Wachsmann-Hogiu S. Direct detection of aptamer-thrombin binding via surface-enhanced Raman spectroscopy. J Biomed Opt. 2010;15(4).

85.He L, Lamont E, Veeregowda B, Sreevatsan S, Haynes CL, Diez-Gonzalez F, et al. Aptamer-based surface-enhanced Raman scattering detection of ricin in liquid foods. Chemical Science. 2011;2(8).

86.Su X, Zhang J, Sun L, Koo TW, Chan S, Sundararajan N, et al. Composite organic-inorganic nanoparticles (COINs) with chemically encoded optical signatures. Nano Lett. 2005;5(1).

87.Sun L, Sung KB, Dentinger C, Lutz B, Nguyen L, Zhang J, et al. Composite organic-inorganic nanoparticles as Raman labels for tissue analysis. Nano Lett. 2007;7(2).

88.Lutz B, Dentinger C, Sun L, Nguyen L, Zhang J, Chmura A, et al. Raman nanoparticle probes for antibody-based protein detection in tissues. The journal of histochemistry and cytochemistry : official journal of the Histochemistry Society. 2008;56(4).

89. Shachaf CM, Elchuri SV, Koh AL, Zhu J, Nguyen LN, Mitchell DJ, et al. A Novel Method for Detection of Phosphorylation in Single Cells by Surface Enhanced Raman Scattering (SERS) using Composite Organic-Inorganic Nanoparticles (COINs). PLoS ONE. 2009;4(4).

90.Guarrotxena N, Bazan GC. Antibody-functionalized SERS tags with improved sensitivity. Chemical communications (Cambridge, England). 2011;47(31).

91.Han XX, Kitahama Y, Itoh T, Wang CX, Zhao B, Ozaki Y. Proteinmediated sandwich strategy for surface-enhanced Raman scattering: application to versatile protein detection. Analytical chemistry. 2009;81(9).

92.Jun BH, Kim JH, Park H, Kim JS, Yu KN, Lee SM, et al. Surface-enhanced Raman spectroscopic-encoded beads for multiplex immunoassay. Journal of combinatorial chemistry. 2007;9(2). 
93. Kim JH, Kang H, Kim S, Jun BH, Kang T, Chae J, et al. Encoding peptide sequences with surface-enhanced Raman spectroscopic nanoparticles. Chemical communications. 2011;47(8).

94.Ong YH, Lim M, Liu Q. Comparison of principal component analysis and biochemical component analysis in Raman spectroscopy for the discrimination of apoptosis and necrosis in K562 leukemia cells. Optics express. 2012;20(20).

95.Rhee H, June YG, Kim ZH, Jeon SJ, Cho M. Phase sensitive detection of vibrational optical activity free-induction-decay: vibrational CD and ORD. $J$ Opt Soc Am B. 2009;26(5).

96.Jun BH, Kim G, Baek J, Kang H, Kim T, Hyeon T, et al. Magnetic field induced aggregation of nanoparticles for sensitive molecular detection. Physical chemistry chemical physics : PCCP. 2011;13(16).

97.Chen L, Hong W, Guo Z, Sa Y, Wang X, Jung YM, et al. Magnetic assistance highly sensitive protein assay based on surface-enhanced resonance Raman scattering. Journal of colloid and interface science. 2012;368(1).

98.He L, Rodda T, Haynes CL, Deschaines T, Strother T, Diez-Gonzalez F, et al. Detection of a foreign protein in milk using surface-enhanced Raman spectroscopy coupled with antibody-modified silver dendrites. Analytical chemistry. 2011;83(5).

99.Combs ZA, Chang S, Clark T, Singamaneni S, Anderson KD, Tsukruk VV. Label-Free Raman Mapping of Surface Distribution of Protein A and IgG Biomolecules. Langmuir. 2011;27(6).

100.Wang M, Jing N, Chou IH, Cote GL, Kameoka J. An optofluidic device for surface enhanced Raman spectroscopy. Lab Chip. 2007;7(5).

101.Chou IH, Benford M, Beier HT, Cote GL, Wang M, Jing N, et al. Nanofluidic biosensing for beta-amyloid detection using surface enhanced Raman spectroscopy. Nano Lett. 2008;8(6).

102.Du Y, Cropek D, Kaazempur Mofrad MR, Weinberg EJ, Khademhosseini A, Borenstein J. Microfluidic Systems for Engineering Vascularized Tissue Constructs. In: Tian W-C, Finehout E, editors. Microfluidics for Biological Applications. New York, NY: Springer US; 2009. p. 223-40.

103.Sivanesan A, Kalaivani G, Fischer A, Stiba K, Leimkuhler S, Weidinger IM. Complementary surface-enhanced resonance Raman spectroscopic biodetection of mixed protein solutions by chitosan- and silica-coated plasmontuned silver nanoparticles. Analytical chemistry. 2012;84(13).

104.Yang X, Gu C, Qian F, Li Y, Zhang JZ. Highly sensitive detection of proteins and bacteria in aqueous solution using surface-enhanced Raman scattering and optical fibers. Analytical chemistry. 2011;83(15). 
105.Han XX, Huang GG, Zhao B, Ozaki Y. Label-free highly sensitive detection of proteins in aqueous solutions using surface-enhanced Raman scattering. Analytical chemistry. 2009;81(9).

106.Zhou Z, Han XX, Huang GG, Ozaki Y. Label-free detection of binary mixtures of proteins using surface-enhanced Raman scattering. Journal of Raman Spectroscopy. 2012;43(6).

107.Keskin S, Culha M. Label-free detection of proteins from dried-suspended droplets using surface enhanced Raman scattering. The Analyst. 2012;137(11).

108.Çulha M, Altunbek M, Keskin S, Saatçi D, editors. Manipulation of silver nanoparticles in a droplet for label-free detection of biological molecules using surface-enhanced Raman scattering. Proc SPIE 7911, Plasmonics in Biology and Medicine VIII 791102; 2011 January 22 - February 18; San Francisco, California.

109.Kahraman M, Yazici MM, Sahin F, Culha M. Convective assembly of bacteria for surface-enhanced Raman scattering. Langmuir : the ACS journal of surfaces and colloids. 2008;24(3).

110.Kahraman M, Sur I, Culha M. Label-Free Detection of Proteins from SelfAssembled Protein-Silver Nanoparticle Structures using Surface-Enhanced Raman Scattering. Analytical chemistry. 2010;82(18).

111.Keskin S, Kahraman M, Culha M. Differential separation of protein mixtures using convective assembly and label-free detection with surface enhanced Raman scattering. Chemical communications. 2011;47(12).

112.Kahraman M, Balz BN, Wachsmann-Hogiu S. Hydrophobicity-driven selfassembly of protein and silver nanoparticles for protein detection using surfaceenhanced Raman scattering. The Analyst. 2013;138(10).

113.Han XX, Jia HY, Wang YF, Lu ZC, Wang CX, Xu WQ, et al. Analytical technique for label-free multi-protein detection based on Western blot and surface-enhanced Raman scattering. Analytical chemistry. 2008;80(8).

114.Abu-Absi NR, Kenty BM, Cuellar ME, Borys MC, Sakhamuri S, Strachan DJ, et al. Real time monitoring of multiple parameters in mammalian cell culture bioreactors using an in-line Raman spectroscopy probe. Biotechnology and bioengineering. 2011;108(5).

115. Whelan J, Craven S, Glennon B. In situ Raman spectroscopy for simultaneous monitoring of multiple process parameters in mammalian cell culture bioreactors. Biotechnology Progress. 2012;28(5).

116.Becker T, Hitzmann B, Muffler K, Pörtner R, Reardon K, Stahl F, et al. Future Aspects of Bioprocess Monitoring. In: Ulber R, Sell D, editors. White 
Biotechnology. Advances in Biochemical Engineering/Biotechnology. 105. New York, NY: Springer Berlin Heidelberg; 2007. p. 249-93.

117.Kusuzawa S, Honda T, Fukata Y, Fukata M, Kanatani S, Tanaka DH, et al. Leucine-rich glioma inactivated 1 (Lgi1), an epilepsy-related secreted protein, has a nuclear localization signal and localizes to both the cytoplasm and the nucleus of the caudal ganglionic eminence neurons. Eur J Neurosci. 2012;36(3).

118.Melendez J, Patel M, Oakes BL, Xu P, Morton P, McClean MN. Real-time optogenetic control of intracellular protein concentration in microbial cell cultures. Integr Biol. 2014;6(3).

119.Vissers JP, Langridge JI, Aerts JM. Analysis and quantification of diagnostic serum markers and protein signatures for Gaucher disease. Mol Cell Proteomics. 2007;6(5).

120.Vermeire S, Van Assche G, Rutgeerts P. C-reactive protein as a marker for inflammatory bowel disease. Inflamm Bowel Dis. 2004;10(5).

121.Malati T. Tumour markers: An overview. Indian J Clin Biochem. 2007;22(2).

122.Lim M, Ye H, Panoskaltsis N, Drakakis EM, Yue XC, Cass AEG, et al. Intelligent bioprocessing for haemotopoietic cell cultures using monitoring and design of experiments. Biotechnology Advances. 2007;25(4).

123.Placzek MR, Chung IM, Macedo HM, Ismail S, Mortera Blanco T, Lim M, et al. Stem cell bioprocessing: fundamentals and principles. $J R$ Soc Interface. 2009;6(32).

124.Wang G, Park HY, Lipert RJ, Porter MD. Mixed monolayers on gold nanoparticle labels for multiplexed surface-enhanced Raman scattering based immunoassays. Anal Chem. 2009;81(23).

125.Fabris L, Schierhorn M, Moskovits M, Bazan GC. Aptatag-based multiplexed assay for protein detection by surface-enhanced Raman spectroscopy. Small. 2010;6(14).

126.Su X, Zhang J, Sun L, Koo T-W, Chan S, Sundararajan N, et al. Composite Organic-Inorganic Nanoparticles (COINs) with Chemically Encoded Optical Signatures. Nano Lett. 2004;5(1).

127.Jun BH, Kim JH, Park H, Kim JS, Yu KN, Lee SM, et al. Surfaceenhanced Raman spectroscopic-encoded beads for multiplex immunoassay. $J$ Comb Chem. 2007;9(2).

128.Perlaki CM, Liu Q, Lim M. Raman Spectroscopy Based Techniques in Tissue Engineering-An Overview. Applied Spectroscopy Reviews. 2013;49(7). 
129. Sivanesan A, Kalaivani G, Fischer A, Stiba K, Leimkuhler S, Weidinger IM. Complementary surface-enhanced resonance Raman spectroscopic biodetection of mixed protein solutions by chitosan- and silica-coated plasmontuned silver nanoparticles. Anal Chem. 2012;84(13).

130.Yang X, Gu C, Qian F, Li Y, Zhang JZ. Highly sensitive detection of proteins and bacteria in aqueous solution using surface-enhanced Raman scattering and optical fibers. Anal Chem. 2011;83(15).

131.Long DA. The Raman effect: a unified treatment of the theory of Raman scattering by molecules. New York: Wiley; 2002.

132. Thomas GJ, Jr. Raman spectroscopy of protein and nucleic acid assemblies. Annu Rev Biophys Biomol Struct. 1999;28(1).

133.Zhao J, McCreery RL. Polarized Raman Spectroscopy of Metallophthalocyanine Monolayers on Carbon Surfaces. Langmuir. 1995;11(10).

134.Allemand CD. Depolarization Ratio Measurements in Raman Spectrometry. Appl Spectrosc. 1970;24(3).

135. Sathyanarayana DN. Vibrational Spectroscopy: Theory And Applications. New Delhi: New Age International (P) Limited; 2007.

136.Zhang Z-M, Chen S, Liang Y-Z, Liu Z-X, Zhang Q-M, Ding L-X, et al. An intelligent background-correction algorithm for highly fluorescent samples in Raman spectroscopy. J Raman Spectrosc. 2009;41(6).

137.Damodara Rao M, Purnima A, Ramesh DV, Ayyanna C. Purification of $\alpha-$ amylase from Bacillus licheniformis by chromatofocusing and gel filtration chromatography. World Journal of Microbiology and Biotechnology. $2002 ; 18(6)$.

138. Wetter LR, Deutsch HF. Immunological studies on egg white proteins. IV. Immunochemical and physical studies of lysozyme. J Biol Chem. 1951;192(1).

139.Hecht E. Optics. 4th ed. Reading, Mass.: Addison-Wesley; 2002.

140.Abdali S, Johannessen C, Nygaard J, Norbygaard T. Resonance surface enhanced Raman optical activity of myoglobin as a result of optimized resonance surface enhanced Raman scattering conditions. J Phys-Condens Mat. $2007 ; 19(28)$.

141.Barron LD, Bogaard MP, Buckingh.Ad. Raman-Scattering of Circularly Polarized-Light by Optically-Active Molecules. Journal of the American Chemical Society. 1973;95(2).

142.Abdali S, Blanch EW. Surface enhanced Raman optical activity (SEROA). Chemical Society Reviews. 2008;37(5). 
143.Johannessen C, White PC, Abdali S. Resonance raman optical activity and surface enhanced resonance raman optical activity analysis of cytochrome c. $J$ Phys Chem A. 2007;111(32).

144.Kneipp H, Kneipp J, Kneipp K. Surface-enhanced Raman optical activity on adenine in silver colloidal solution. Analytical chemistry. 2006;78(4).

145.Etchegoin PG, Galloway C, Le Ru EC. Polarization-dependent effects in surface-enhanced Raman scattering (SERS). Physical Chemistry Chemical Physics. 2006;8(22).

146.Lee PC, Meisel D. Adsorption and Surface-Enhanced Raman of Dyes on Silver and Gold Sols. J Phys Chem-Us. 1982;86(17).

147.Lombardini R, Acevedo R, Halas NJ, Johnson BR. Plasmonic Enhancement of Raman Optical Activity in Molecules near Metal Nanoshells: Theoretical Comparison of Circular Polarization Methods. The Journal of Physical Chemistry C. 2010;114(16).

148.McClure JP, editor The Schmidt-Czerny-Turner spectrograph2014.

149.Sears FW. Optics. 3d ed. Cambridge, Mass.,: Addison-Wesley; 1949.

150.Spencer KM, Freedman TB, Nafie LA. Scattered Circular-Polarization Raman Optical-Activity. Chem Phys Lett. 1988;149(4).

151.Yamamoto S, Watarai H. Incident circularly polarized Raman optical activity spectrometer based on circularity conversion method. J Raman Spectrosc. 2010;41(12).

152.Schmid F-X. Biological Macromolecules: UV-visible Spectrophotometry. eLS: John Wiley \& Sons, Ltd; 2001.

153. Sharma B, Frontiera RR, Henry AI, Ringe E, Van Duyne RP. SERS: Materials, applications, and the future. Materials Today. 2012;15(1-2).

154. Stewart A, Murray S, Bell SEJ. Simple preparation of positively charged silver nanoparticles for detection of anions by surface-enhanced Raman spectroscopy. The Analyst. 2015;140(9).

155.Leopold N, Lendl B. A new method for fast preparation of highly surfaceenhanced Raman scattering (SERS) active silver colloids at room temperature by reduction of silver nitrate with hydroxylamine hydrochloride. J Phys Chem B. 2003;107(24).

156.Perlaki CM, Liu Q, Lim M. Raman Spectroscopy Based Techniques in Tissue Engineering-An Overview. Applied Spectroscopy Reviews. 2014;49(7). 
157.Lowe AB, McCormick CL. Reversible addition-fragmentation chain transfer (RAFT) radical polymerization and the synthesis of water-soluble (co)polymers under homogeneous conditions in organic and aqueous media. Prog Polym Sci. 2007;32(3).

158.Moad G, Chong YK, Postma A, Rizzardo E, Thang SH. Advances in RAFT polymerization: the synthesis of polymers with defined end-groups. Polymer. 2005;46(19).

159. Bastus NG, Comenge J, Puntes V. Kinetically Controlled Seeded Growth Synthesis of Citrate-Stabilized Gold Nanoparticles of up to $200 \mathrm{~nm}$ : Size Focusing versus Ostwald Ripening. Langmuir. 2011;27(17).

160.Kusolkamabot K, Sae-ung P, Niamnont N, Wongravee K, Sukwattanasinitt M, Hoven VP. Poly(N-isopropylacrylamide)-Stabilized Gold Nanoparticles in Combination with Tricationic Branched Phenylene-Ethynylene Fluorophore for Protein Identification. Langmuir. 2013;29(39).

161.Yusa SI, Fukuda K, Yamamoto T, Iwasaki Y, Watanabe A, Akiyoshi K, et al. Salt effect on the heat-induced association Behavior of gold nanoparticles coated with Poly(N-isopropylacrylamide) prepared via reversible addition Fragmentation chain transfer (RAFT) radical polymerization. Langmuir. 2007;23(26).

162.Geddes CD. Metal-enhanced fluorescence. Hoboken, N.J.: Wiley; 2010.

163.Pagba CV, Lane SM, Cho HS, Wachsmann-Hogiu S. Direct detection of aptamer-thrombin binding via surface-enhanced Raman spectroscopy. Journal of biomedical optics. 2010;15(4).

164. Shrivastava S, Matsuoka H. Photoresponsive Block Copolymer: Synthesis, Characterization, and Surface Activity Control. Langmuir. 2014;30(14).

165.Jochum FD, zur Borg L, Roth PJ, Theato P. Thermo- and Light-Responsive Polymers Containing Photoswitchable Azobenzene End Groups.

Macromolecules. 2009;42(20).

166.Alkilany AM, Abulateefeh SR, Mills KK, Yaseen AIB, Hamaly MA, Alkhatib HS, et al. Colloidal Stability of Citrate and Mercaptoacetic Acid Capped Gold Nanoparticles upon Lyophilization: Effect of Capping Ligand Attachment and Type of Cryoprotectants. Langmuir. 2014;30(46).

167.Kneipp K, Kneipp H, Itzkan I, Dasari RR, Feld MS. Ultrasensitive chemical analysis by Raman spectroscopy. Chem Rev. 1999;99(10).

168.Hecht L, Barron LD, Gargaro AR, Wen ZQ, Hug W. Raman OpticalActivity Instrument for Biochemical-Studies. J Raman Spectrosc. 1992;23(7).

169. Nafie LA. Infrared and Raman vibrational optical activity of biomolecules. Spectroscopy of Biological Molecules. 1995. 
170.Kistemaker JCM, Stacko P, Visser J, Feringa BL. Unidirectional rotary motion in achiral molecular motors. Nat Chem. 2015;7(11).

171.Combs ZA, Chang S, Clark T, Singamaneni S, Anderson KD, Tsukruk VV. Label-free Raman mapping of surface distribution of protein a and $\mathrm{IgG}$ biomolecules. Langmuir. 2011;27(6).

172.Dick LA, McFarland AD, Haynes CL, Van Duyne RP. Metal Film over Nanosphere (MFON) Electrodes for Surface-Enhanced Raman Spectroscopy (SERS): Improvements in Surface Nanostructure Stability and Suppression of Irreversible Loss. The Journal of Physical Chemistry B. 2002;106(4).

173.Félidj N, Aubard J, Lévi G, Krenn JR, Hohenau A, Schider G, et al. Optimized surface-enhanced Raman scattering on gold nanoparticle arrays. Appl Phys Lett. 2003;82(18).

174.Liu H, Schwenke AM, Kretschmer F, Hoeppener S, Schubert US. Gold Nanoparticle Cluster Arrays for High-Performance SERS Substrates Fabricated by Electro-oxidative Lithography. ChemNanoMat. 2016. 